\title{
Polymer Membranes for All-Vanadium Redox Flow Batteries: A Review
}

\author{
Dennis Düerkop ${ }^{1, *(\mathbb{D}}$, Hartmut Widdecke ${ }^{1}$, Carsten Schilde ${ }^{2}$, Ulrich Kunz ${ }^{3}$ and Achim Schmiemann ${ }^{1}$ \\ 1 Institute of Recycling, Ostfalia University of Applied Sciences, Robert-Koch-Platz 8a, \\ 38440 Wolfsburg, Germany; h.widdecke@ostfalia.de (H.W.); a.schmiemann@ostfalia.de (A.S.) \\ 2 Institute of Particle Technology, Braunschweig University of Technology, Volkmaroder Straße 5, \\ 38100 Braunschweig, Germany; c.schilde@tu-bs.de \\ 3 Institute of Chemical and Electrochemical Process Engineering, Clausthal University of Technology, \\ Leibnizstraße 17, 38678 Clausthal-Zellerfeld, Germany; kunz@icvt.tu-clausthal.de \\ * Correspondence: den.dueerkop@ostfalia.de
}

check for

updates

Citation: Düerkop, D.; Widdecke, H.; Schilde, C.; Kunz, U.; Schmiemann, A. Polymer Membranes for All-

Vanadium Redox Flow Batteries: A Review. Membranes 2021, 11, 214. https://doi.org/10.3390/ membranes11030214

Academic Editor: Mohammad K. Hassan

Received: 25 January 2021

Accepted: 5 March 2021

Published: 18 March 2021

Publisher's Note: MDPI stays neutral with regard to jurisdictional claims in published maps and institutional affiliations.

Copyright: (c) 2021 by the authors. Licensee MDPI, Basel, Switzerland. This article is an open access article distributed under the terms and conditions of the Creative Commons Attribution (CC BY) license (https:/ / creativecommons.org/licenses/by/ $4.0 /)$.

\begin{abstract}
Redox flow batteries such as the all-vanadium redox flow battery (VRFB) are a technical solution for storing fluctuating renewable energies on a large scale. The optimization of cells regarding performance, cycle stability as well as cost reduction are the main areas of research which aim to enable more environmentally friendly energy conversion, especially for stationary applications. As a critical component of the electrochemical cell, the membrane influences battery performance, cycle stability, initial investment and maintenance costs. This review provides an overview about flow-battery targeted membranes in the past years (1995-2020). More than 200 membrane samples are sorted into fluoro-carbons, hydro-carbons or N-heterocycles according to the basic polymer used. Furthermore, the common description in membrane technology regarding the membrane structure is applied, whereby the samples are categorized as dense homogeneous, dense heterogeneous, symmetrical or asymmetrically porous. Moreover, these properties as well as the efficiencies achieved from VRFB cycling tests are discussed, e.g., membrane samples of fluoro-carbons, hydro-carbons and N-heterocycles as a function of current density. Membrane properties taken into consideration include membrane thickness, ion-exchange capacity, water uptake and vanadium-ion diffusion. The data on cycle stability and costs of commercial membranes, as well as membrane developments, are compared. Overall, this investigation shows that dense anion-exchange membranes (AEM) and N-heterocycle-based membranes, especially poly(benzimidazole) (PBI) membranes, are suitable for VRFB requiring low self-discharge. Symmetric and asymmetric porous membranes, as well as cation-exchange membranes (CEM) enable VRFB operation at high current densities. Amphoteric ion-exchange membranes (AIEM) and dense heterogeneous CEM are the choice for operation mode with the highest energy efficiency.
\end{abstract}

Keywords: all-vanadium redox flow battery; polymer membrane; efficiency; current density; costs

\section{Table of Contents}

1. Introduction 2

2. Commercial membranes for VRFB Work 5

3. Polymer membrane Development 9 3.1 Membrane Structures 9

$\begin{array}{ll}3.2 \text { Membrane Polymers } & 10\end{array}$

4. Polymer membrane Development 11 4.1 Membrane Properties $\quad 15$

4.2 Membrane Impact on VRFB Cell Performance 32

5. Cycle Stability 46

6. Membrane Costs 47

7. Conclusions 48

8. Acknowledgements 50

9. Abbreviations 51

10. References $\quad 52$ 


\section{Introduction}

The growing use of renewable energy supplements fossil fuel and nuclear power in many parts of the world with considerable proportions [1,2]. The expansion of the electrical grid and the efficient use of fluctuating energies still pose challenges, which are fundamentally associated with costs. In addition to lithium-ion batteries, flow-batteries have increasingly gained interest. Redox flow batteries have external tanks to store electric energy in vanadium-based electrolytes. The electrolytes are pumped through the battery stack for energy conversion (charging or discharging). This is the main advantage of flow batteries. The power depends on the stack size and the capacity on the volume of the tanks.

The intermediate storage of electrical energy in all-vanadium redox flow batteries (Figure 1) is being pursued seriously and is demonstrated in numerous pilot projects and industrial installations (e.g., cellcube [3], Rongke Power [4], Sumitomo Electric [5], Fraunhofer ICT [6]). These highly efficient electrochemical storage units can generally be industrially manufactured in large quantities [7-9]. Its intended use can be either as part of large-scale plants or for consumer use in smaller applications. Studies on techno-economic assessment of VRFB are analyzed in [10]. As a result, guide values of $650 \mathrm{EUR}(\mathrm{kWh})^{-1}$ and 550 EUR $(\mathrm{kWh})^{-1}$ for VRFB systems in a power range of $10-1000 \mathrm{~kW}$ providing electrical energy for $4 \mathrm{~h}$ and $8 \mathrm{~h}$ are derived from literature. Here, the key components of the electrochemical cell, the active species vanadium, the membranes, the electrode felts and bipolar plates differ in proportion to the total system costs. The proportion of Vanadium costs, membrane costs and electrode felt/bipolar plate costs to total system costs is about $30-60 \%, 3-30 \%$ and below $5 \%$.
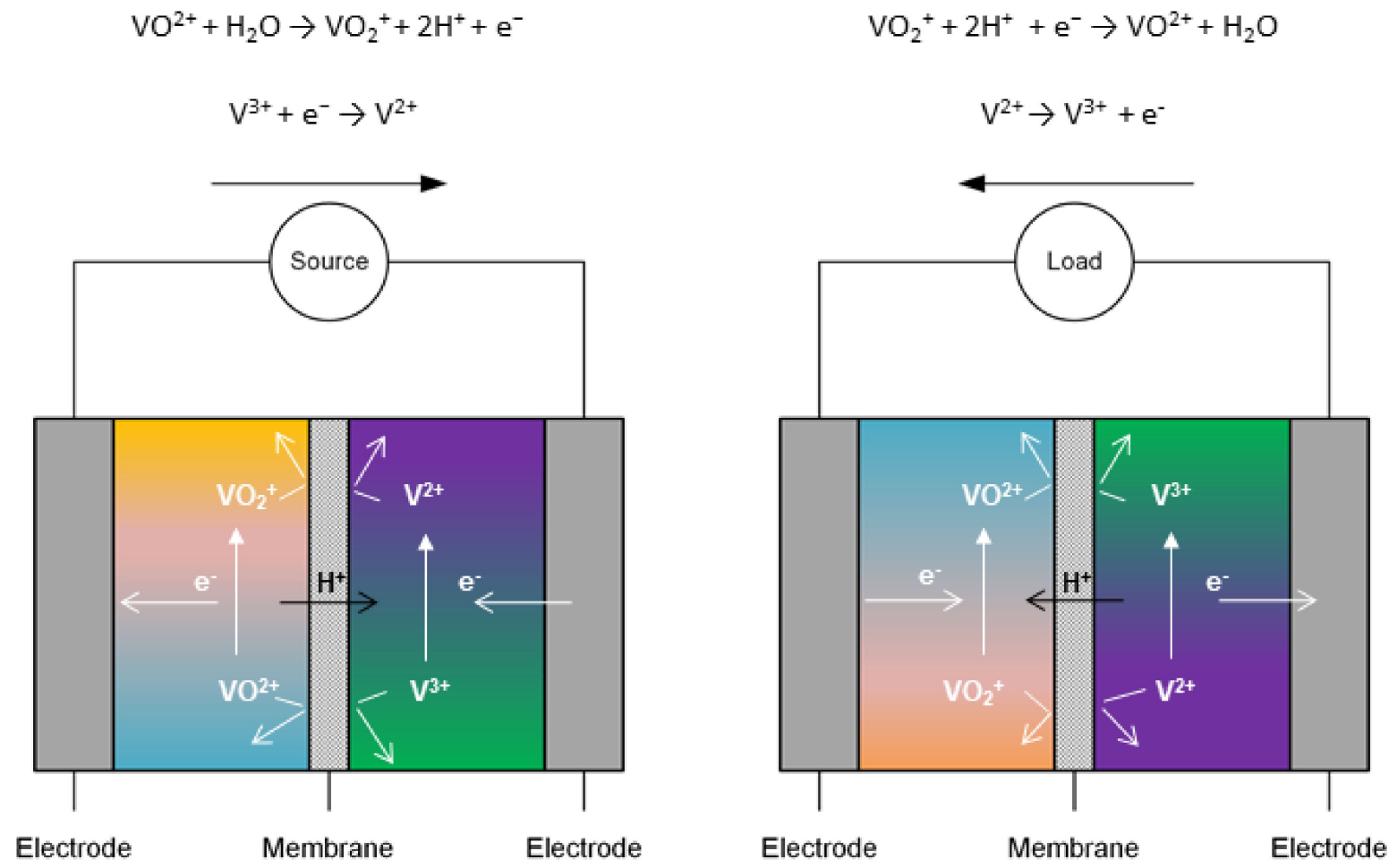

Figure 1. Schematic of the all-Vanadium redox flow battery (left charging/right discharging).

Cost reduction is one way to realize marketable products. As an integral part of the VRFB, the membrane influences investment costs, service life and battery performance. Perfluorosulfonic acid (PFSA) membranes account for about $40 \%$ of the investment costs of a VRFB stack. Like other VRFB components, the membrane influences the efficiency and power of the cells. Looking at membrane development as a way to optimize costs, these could be reduced by making cheaper membranes available on the market. The costs 
consist of the specific cost of raw materials, the manufacturing process and, in particular, on the quantity produced.

Generally, high proton conductivity and high $\mathrm{H}^{+} / \mathrm{V}$ selectivity are the main issues to overcome in designing membranes for VRFB. Mechanical and chemical stability (resistance to the highly oxidizing electrolyte of the positive half-cell) during VRFB operation are the main issues membrane materials have to overcome.

The development of membrane materials for VRFB has been an ongoing process for decades. From 2011 to 2020, several review papers were published summarizing the most important membrane developments. In [11] Li et al. describe the basic properties of VRFB and its development history. The first demonstration projects are mentioned and the electrical performances and storage capacities achieved are listed. For ion-exchange membranes, requirements and parameters relevant for the operation of the VRFB are described. The production of membranes is also briefly discussed. Furthermore, membrane developments published over a period of about 10 years are listed, differentiating between the various material groups: pore filled membranes; perfluorinated membranes; modified perfluorinated membranes; partially fluorinated IEMs and non-fluorinated membranes. In [12], an overview of membrane properties that are relevant for the VRFB is given. Furthermore, membrane types that can be used in the VRFB are summarized. They are divided into cation-exchange membranes, anion-exchange membranes, amphoteric membranes and non-ionic membranes. H. Prifti et al. [13] provide a brief introduction to the design, manufacture and characterization of ion-exchange membranes. The modification of membranes to improve VRFB performance is discussed and various examples are given. H. H. Cha [14] summarizes the efforts to develop nanocomposite membranes for VRFB. The developments focus on the reduction of vanadium-ion permeability, the improvement of proton conductivity for improved battery performance and a long service life of the battery systems. The focus is on functionalized materials for nanocomposite membranes. The description of membrane properties and the calculation of the coulombic, voltage and energy efficiency are described in detail in previous reviews $[11,13,15,16]$ and are not discussed further here. Table 1 gives an overview of published review papers considering important membrane properties and membranes for redox flow batteries.

Table 1. Overview of review papers considering vanadium redox flow battery membranes.

\begin{tabular}{|c|c|c|c|c|}
\hline Year & Journal & Title & Main Focus & Ref. \\
\hline 2011 & $\begin{array}{l}\text { Energy Environ. } \\
\text { Sci. }\end{array}$ & $\begin{array}{l}\text { Ion exchange membranes for vanadium } \\
\text { redox flow battery (VRB) application }\end{array}$ & $\begin{array}{l}\text { all aspects related to IEMs } \\
\text { that are of relevance to } \\
\text { understand IEMs for VRFB }\end{array}$ & {$[11]$} \\
\hline 2011 & ChemSusChem & $\begin{array}{l}\text { Membrane Development for Vanadium } \\
\text { Redox Flow Batteries }\end{array}$ & $\begin{array}{c}\text { basic scientific issues } \\
\text { associated with membrane } \\
\text { use in VRFBs }\end{array}$ & [12] \\
\hline 2012 & Membranes & Membranes for VRFB Applications & $\begin{array}{c}\text { membranes for } \\
\text { all-vanadium redox flow } \\
\text { battery which has received the most } \\
\text { attention. }\end{array}$ & [13] \\
\hline 2013 & Electrochimica Acta & $\begin{array}{l}\text { Review of material research and } \\
\text { development for vanadium redox flow } \\
\text { battery applications }\end{array}$ & $\begin{array}{l}\text { a historical overview of } \\
\text { materials research and } \\
\text { development }\end{array}$ & [15] \\
\hline 2014 & $\begin{array}{l}\text { Energy Environ. } \\
\text { Sci. }\end{array}$ & $\begin{array}{l}\text { Anion-exchange membranes in } \\
\text { electrochemical energy systems }\end{array}$ & $\begin{array}{l}\text { technological and scientific limitations } \\
\text { and the future challenges related to } \\
\text { the use of anion-exchange membranes }\end{array}$ & [16] \\
\hline 2015 & J.o.Nanomaterials & $\begin{array}{l}\text { Recent development of Nanocomposite } \\
\text { Membranes for Vanadium redox Flow } \\
\text { Batteries }\end{array}$ & $\begin{array}{l}\text { efforts in developing } \\
\text { nanocomposite membranes }\end{array}$ & [14] \\
\hline
\end{tabular}


Table 1. Cont.

\begin{tabular}{|c|c|c|c|c|}
\hline Year & Journal & Title & Main Focus & Ref. \\
\hline 2015 & RSC Adv. & $\begin{array}{c}\text { Recent development of polymer } \\
\text { membranes as separators for all-vanadium } \\
\text { redox flow batteries }\end{array}$ & $\begin{array}{c}\text { new cation exchange } \\
\text { membranes, anion exchange } \\
\text { membranes, amphoteric ionexchange } \\
\text { membranes, } \\
\text { and non-ionic porous } \\
\text { materials }\end{array}$ & [17] \\
\hline 2015 & RSC Adv. & $\begin{array}{c}\text { A review on recent developments of anion } \\
\text { exchange membranes for fuel cells and } \\
\text { redox flow batteries }\end{array}$ & $\begin{array}{l}\text { developments in the } \\
\text { synthesis and applications of } \\
\text { AEMs in the field of } \\
\text { electrochemical energy } \\
\text { conversion and storage }\end{array}$ & [18] \\
\hline 2017 & Chem. Soc. Rev. & $\begin{array}{l}\text { Porous membranes in secondary battery } \\
\text { technologies }\end{array}$ & $\begin{array}{l}\text { understanding of the } \\
\text { preparation-structure- } \\
\text { performance relationship }\end{array}$ & [19] \\
\hline 2017 & $\begin{array}{l}\text { Journal of } \\
\text { Membrane Science }\end{array}$ & $\begin{array}{l}\text { Ion exchange membranes: New } \\
\text { developments and applications }\end{array}$ & new iem materials & [20] \\
\hline 2018 & Chem. Commun. & $\begin{array}{l}\text { Ion conducting membranes for aqueous } \\
\text { flow battery systems }\end{array}$ & $\begin{array}{l}\text { porous membranes, different } \\
\text { flow batteries }\end{array}$ & [21] \\
\hline 2018 & $\begin{array}{l}\text { Energy Environ. } \\
\text { Sci. }\end{array}$ & $\begin{array}{c}\text { Review of electrical energy storage } \\
\text { technologies, materials and systems: } \\
\text { challenges and prospects for large-scale } \\
\text { grid storage }\end{array}$ & $\begin{array}{l}\text { status and options for mechanical, } \\
\text { thermal, electrochemical, and } \\
\text { chemical energy storage }\end{array}$ & [1] \\
\hline 2018 & $\begin{array}{c}\text { Journal of } \\
\text { Membrane Science }\end{array}$ & $\begin{array}{c}\text { Selectivity of ion exchange membranes: A } \\
\text { review }\end{array}$ & $\begin{array}{l}\text { selectivity of ion exchange } \\
\text { membranes }\end{array}$ & [22] \\
\hline 2019 & Applied Energy & $\begin{array}{c}\text { Recent development of membrane for } \\
\text { vanadium redox flow battery applications: } \\
\text { A review }\end{array}$ & $\begin{array}{l}\text { research on membranes for } \\
\text { VRFB }\end{array}$ & [23] \\
\hline 2019 & $\begin{array}{l}\text { Current Opinion in } \\
\text { Electrochemistry }\end{array}$ & $\begin{array}{l}\text { Membranes and separators for redox flow } \\
\text { batteries }\end{array}$ & $\begin{array}{l}\text { current development trends for } \\
\text { membranes and separators for VRFB }\end{array}$ & [24] \\
\hline 2020 & $\begin{array}{l}\text { Journal of Energy } \\
\text { Storage }\end{array}$ & $\begin{array}{l}\text { Membranes for all vanadium redox flow } \\
\text { batteries }\end{array}$ & $\begin{array}{l}\text { different membrane types, } \\
\text { membrane performance }\end{array}$ & [25] \\
\hline 2021 & Membranes & $\begin{array}{l}\text { Polymer Membranes for all-Vanadium } \\
\text { Redox Flow Batteries: A Review }\end{array}$ & $\begin{array}{c}\text { graphical overview of polymer } \\
\text { membranes; main polymer, impact } \\
\text { on VRFB }\end{array}$ & $\begin{array}{l}\text { this } \\
\text { paper }\end{array}$ \\
\hline
\end{tabular}

Publications of new membranes and their respective tests in VRFB cells have increased significantly since 2012. In addition to the information provided in [11-14] further developments have been added to the range of membrane types described above. In this review, the basic classification of the membranes into fluoro-carbon, hydro-carbon and $\mathrm{N}$-heterocycle-based membranes is made. They are additionally classified according to their structure and indicated by their chemical character as CEM, AEM, AIEM or non-ionic.

The main component of this review is a digest of relevant research results from previous years, too. Thereby, the focus is on membrane main polymers and the impact of new membranes on VRFB performances. Especially, the graphical presentation of the data should provide a comprehensive overview of achievable performance for VRFB cells using different membranes. Knowing that the performance of a VRFB cell is not exclusively dependent on the membrane used, the results are displayed next to each other in order to provide a simplified overview of different membrane types.

\section{Commercial Membranes for VRFB}

Various companies offer ion-exchange membranes, shown in Table 2, for use in VRFB. Here, the coulombic, voltage and energy efficiencies (CE, VE and EE) are indicated for the 
respective current densities (CD). These membranes differ in their application for different operating modes, which are divided into energy efficient operation, operation with high current densities and operation with low self-discharge [26]. The "Vanadion" membrane was developed on the basis of Nafion. A thin, more selective Nafion layer was applied to a microporous layer. The membrane is $230 \mu \mathrm{m}$ thick and provides a constant energy efficiency over 80 charge/discharge cycles [27]. The Nafion membranes N212, N115 and N117 have been commercially available for many years and are often used as reference membranes in comparison to newly developed membrane samples (MS). The measurement results from many studies regarding VRFB efficiencies can be found in Table 3 and Figure 2. More than two decades ago Asahi Glass Co. Ltd. has developed hydro-carbon-based anion exchange membranes with improved chemical stability in VRFB [28,29]. This resulted in the oxidation-stabilized AEM Selemion ${ }^{\mathrm{TM}}$ APS4 membrane, which is distributed by AGC [30]. Solvay offered the short side chain PFSA membrane known as Aquivion, which was successfully tested in VRFB [31]. It should be noted that due to different test conditions, the respective efficiency results for the membranes used cannot be compared directly with each other.

Table 2. Overview of commercial membranes for VRFB: (1-9) FumaTech, (10-14) DuPont, (15, 16) Asahi Glass, (17) Solvay.

\begin{tabular}{|c|c|c|c|c|c|c|c|c|}
\hline \multirow{2}{*}{ MS } & \multirow{2}{*}{ Membrane } & \multirow{2}{*}{ Chem } & \multirow{2}{*}{ Operating Mode } & \multirow{2}{*}{$\frac{\mathrm{CD}}{\mathrm{mA} \mathrm{cm}-2}$} & \multirow{2}{*}{$\begin{array}{c}\text { CE } \\
\%\end{array}$} & \multirow{2}{*}{$\begin{array}{c}\text { VE } \\
\%\end{array}$} & \multirow{2}{*}{$\begin{array}{l}\text { EE } \\
\%\end{array}$} & \multirow{2}{*}{ Ref. } \\
\hline & & & & & & & & \\
\hline 1 & FAP-330-PE & AEM & high current density & $20-80$ & 95.9 & 94.4 & 90.5 & [26] \\
\hline 2 & FAP-450 & AEM & high energy efficiency & $20-80$ & 98 & 90.8 & 89 & [26] \\
\hline 3 & FAP-375-PP & AEM & low self-discharging & $20-80$ & 99 & 89.9 & 89 & [26] \\
\hline 4 & FS-930 & CEM & high current density & $20-80$ & 96 & 94.8 & 91 & [26] \\
\hline 5 & F-930-RFD & CEM & high current density & $20-80$ & 98.5 & 92.4 & 91 & [26] \\
\hline 6 & F-1075-PK & CEM & low self-discharging & $20-80$ & 99.5 & 90.5 & 90 & [26] \\
\hline 7 & F-1850 & CEM & low self-discharging & $20-80$ & 99.5 & 83.4 & 83 & [26] \\
\hline 8 & VX-20 & AEM & low self-discharging & 80 & 99.99 & 84 & 84 & [32] \\
\hline 9 & Fumapem 14,100 & CEM & - & 40 & 91.3 & 90.2 & 82.4 & [33] \\
\hline 10 & Vanadion & CEM & high current density & 80 & 88 & 92 & 81 & [27] \\
\hline 11 & Vanadion & CEM & high current density & 320 & 96 & 76 & 73 & [27] \\
\hline 12 & Nafion N117 & CEM & high current density & 100 & 96 & 61 & 59 & [34] \\
\hline 13 & Nafion N115 & CEM & high current density & 80 & 95 & 90 & 86 & [27] \\
\hline 14 & Nafion 212 & CEM & high current density & 200 & 97.6 & 77.9 & 76 & [35] \\
\hline 15 & New Selemion & AEM & - & 40 & 98.6 & 87.5 & 86.3 & [28] \\
\hline 16 & New Selemion CL & AEM & - & 60 & 93.5 & 87.7 & 82 & [29] \\
\hline 17 & Aquivion-E87 & CEM & high current density & 80 & 97 & 86 & 83 & [31] \\
\hline
\end{tabular}

In VRFB cells, Nafion is suitable as a reference membrane due to its known properties in electrochemical cells and its worldwide availability. Nafion, for example, is preferably used to show the change in battery performance caused by changing the membrane component. Table 3 lists efficiencies measured with different Nafion types as reference membranes for comparison measurements in VRFB cells. N212, N115 and N117 were used in the cycling tests at different current densities. B. Jang et al. [36] describe the influence of Nafion membrane pretreatment on battery performance.

The tabular listing of the results shows how efficiencies under different test conditions vary. The lowest energy efficiency of 51\% is achieved with N115 (MS34) and the highest energy efficiency of $92 \%$ with N212 (MS19).

Figure 2a shows the current density used in VRFB cycling experiments with Nafion membranes and the year in which the measurements were published. The numbers close to the data points refer to the sample numbers of the membranes in Table 3. It can be seen that from 2012 onwards cycling tests could increasingly be carried out at current densities greater than $100 \mathrm{~mA} \mathrm{~cm}^{-2}$. With $\mathrm{N} 212$ tests up to a current density of $240 \mathrm{~mA} \mathrm{~cm}^{-2}$, with N115 tests with a current density up to $320 \mathrm{~mA} \mathrm{~cm}^{-2}$ and with N117 tests with a current density up to $260 \mathrm{~mA} \mathrm{~cm}^{-2}$ were performed. This means that progress has been made 
through cell development in recent years and the research potential does not yet seem to be exhausted.
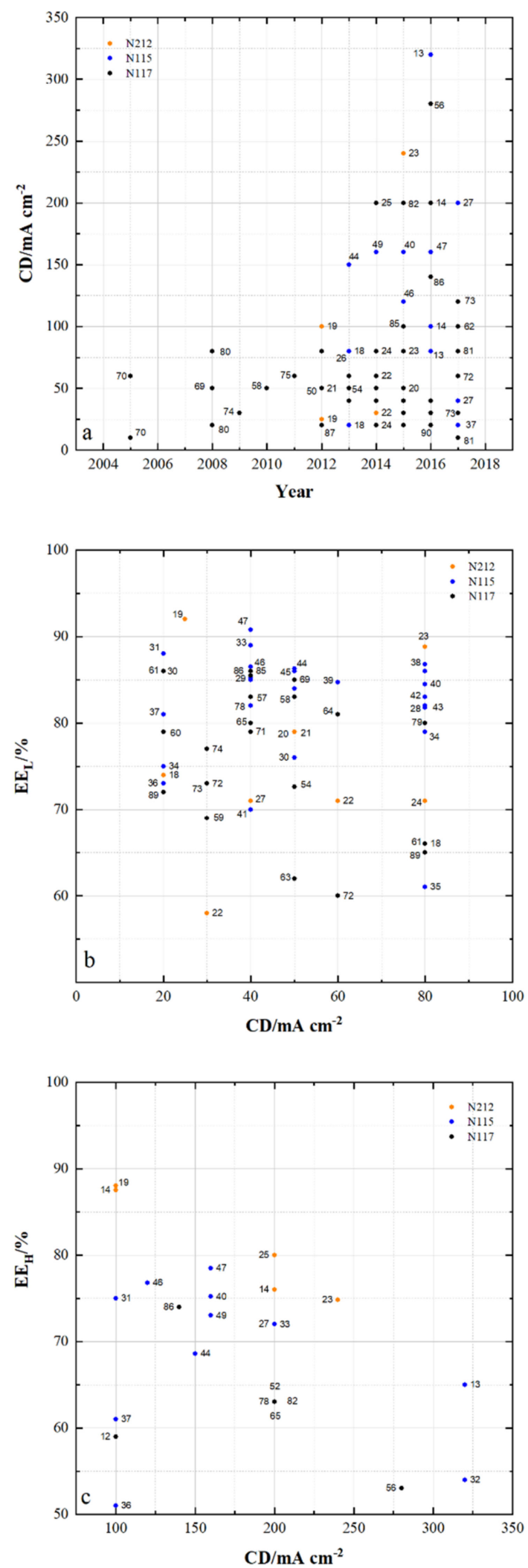

Figure 2. Results from VRFB tests using Nafion: (a) current density during cycling tests (b) energy efficiency of VRFB cells at current densities $<100 \mathrm{~mA} \mathrm{~cm}^{-2}$ and (c) energy efficiency of VRFB cells at current densities $\geq 100 \mathrm{~mA} \mathrm{~cm}^{-2}$ 
Table 3. The efficiencies of VRFB cells using the listed reference membranes N212, N115 and N117 for investigated current densities.

\begin{tabular}{|c|c|c|c|c|c|c|c|c|c|c|c|c|c|}
\hline \multirow{2}{*}{ MS } & \multirow{2}{*}{ Membrane } & \multirow{2}{*}{$\frac{\mathrm{CD}}{\mathrm{mA} \mathrm{cm}^{-2}}$} & \multirow{2}{*}{$\begin{array}{c}\mathrm{CE} \\
\%\end{array}$} & \multirow{2}{*}{$\begin{array}{l}\text { VE } \\
\%\end{array}$} & \multirow{2}{*}{$\begin{array}{c}\mathrm{EE} \\
\%\end{array}$} & \multirow{2}{*}{ Ref. } & \multirow{2}{*}{ MS } & \multirow{2}{*}{ Membrane } & \multirow{2}{*}{$\frac{\mathrm{CD}}{\mathrm{mA} \mathrm{cm}^{-2}}$} & \multirow{2}{*}{$\begin{array}{c}\mathrm{CE} \\
\%\end{array}$} & \multirow{2}{*}{$\begin{array}{l}\text { VE } \\
\%\end{array}$} & \multirow{2}{*}{$\begin{array}{c}\mathrm{EE} \\
\%\end{array}$} & \multirow{2}{*}{ Ref. } \\
\hline & & & & & & & & & & & & & \\
\hline 18 & N212 & 20 & 81.2 & 92 & 74 & {$[37]$} & 12 & N117 & 100 & 96 & 61 & 59 & {$[34]$} \\
\hline 18 & $\mathrm{~N} 212$ & 80 & 94 & 70 & 66 & [37] & 52 & N117 & 40 & 90 & 92 & 83 & [38] \\
\hline 19 & N212 & 25 & 97 & 95 & 92 & [39] & 52 & N117 & 200 & 95 & 66 & 63 & [38] \\
\hline 19 & N212 & 100 & 97 & 91 & 88 & [39] & 53 & N117 & 40 & 93.8 & 90.7 & 85 & [33] \\
\hline 20 & $\mathrm{~N} 212$ & 50 & 92 & 86 & 79 & {$[40]$} & 54 & N117 & 50 & 87.6 & 82.6 & 72.6 & [41] \\
\hline 21 & N212 & 50 & 92 & 86 & 79 & [42] & 55 & N117 & 50 & 96.5 & 91 & 87.5 & [43] \\
\hline 22 & N212 & 30 & 60 & 96 & 58 & {$[44]$} & 56 & N117 & 40 & 91 & 93 & 84 & {$[45]$} \\
\hline 22 & N212 & 60 & 78 & 92.3 & 71 & {$[44]$} & 56 & N117 & 280 & 98 & 54 & 53 & {$[45]$} \\
\hline 23 & $\mathrm{~N} 212$ & 80 & - & - & 88.8 & {$[46]$} & 57 & N117 & 40 & 93 & 89 & 83 & [47] \\
\hline 23 & N212 & 240 & - & - & 74.8 & {$[46]$} & 58 & N117 & 50 & 95 & 87 & 83 & [48] \\
\hline 24 & N212 & 20 & - & - & 81 & {$[49]$} & 59 & N117 & 30 & 90 & 76.6 & 69 & [50] \\
\hline 24 & N212 & 80 & - & - & 71 & [49] & 60 & N117 & 20 & 84 & 94.1 & 79 & [51] \\
\hline 25 & $\mathrm{~N} 212$ & 200 & 91 & 88 & 80 & {$[52]$} & 60 & N117 & 80 & 91 & 81 & 73.5 & [51] \\
\hline 26 & N212 & 80 & 94 & 75 & 71 & {$[53]$} & 61 & N117 & 20 & 81 & 72 & 86 & [54] \\
\hline 27 & N212 & 40 & 75 & 95 & 71 & [55] & 61 & N117 & 80 & 95 & 66 & 66 & [54] \\
\hline 27 & $\mathrm{~N} 212$ & 200 & 92 & 80 & 72 & [55] & 62 & N117 & 100 & 96 & 63 & 60.5 & [56] \\
\hline 14 & N212 & 100 & 95.5 & 91.6 & 87.5 & [35] & 63 & N117 & 20 & 74 & 81 & 67 & [57] \\
\hline \multirow[t]{2}{*}{14} & N212 & 200 & 97.6 & 77.9 & 76 & [35] & 63 & N117 & 50 & 83 & 70 & 62 & [57] \\
\hline & & & & & & & 64 & N117 & 60 & 91 & 89 & 81 & [58] \\
\hline 28 & N115 & 80 & 94.6 & 86.6 & 82 & [59] & 65 & N117 & 40 & 87 & 92 & 80 & [60] \\
\hline 29 & N115 & 40 & 94.5 & 90.1 & 85.2 & [61] & 65 & N117 & 200 & 93 & 68 & 63 & [60] \\
\hline 30 & N115 & 20 & 92.5 & 92.5 & 86 & [62] & 66 & N117 & 30 & 96.4 & 90.7 & 87.4 & [63] \\
\hline 30 & N115 & 50 & 94 & 82.5 & 76 & [62] & 67 & N117 & 20 & 85 & 81 & 68.9 & [64] \\
\hline 31 & N115 & 20 & 94 & 94 & 88 & [65] & 67 & N117 & 80 & 92 & 70 & 64.4 & [64] \\
\hline 31 & N115 & 100 & 97.5 & 77.5 & 75 & [65] & 68 & N117 & 50 & 89.9 & 90.8 & 81.6 & [66] \\
\hline 32 & N115 & 40 & 88 & 94 & 82 & [45] & 69 & N117 & 50 & 93.8 & 90.7 & 85 & [67] \\
\hline 32 & N115 & 320 & 96 & 56 & 54 & [45] & 70 & N117 & 10 & 72.5 & 93.8 & 68 & [68] \\
\hline 33 & N115 & 40 & 94 & 94.7 & 89 & [69] & 70 & N117 & 60 & 89 & 75.3 & 67 & [68] \\
\hline 33 & N115 & 200 & 97.5 & 73.8 & 72 & [69] & 71 & N117 & 40 & 94 & 84 & 79 & [70] \\
\hline 34 & N115 & 20 & 79 & 95 & 75 & [71] & 72 & N117 & 30 & 90 & 81 & 73 & [72] \\
\hline 34 & N115 & 80 & 94 & 84 & 79 & [71] & 72 & N117 & 60 & 94.5 & 63 & 60 & [72] \\
\hline 35 & N115 & 20 & 81 & 91 & 73 & [73] & 73 & N117 & 30 & 95 & 76.8 & 73 & [74] \\
\hline 35 & N115 & 80 & 92 & 66 & 61 & [73] & 73 & N117 & 120 & 97 & 64.4 & 62.5 & [74] \\
\hline 36 & N115 & 20 & 90 & 81 & 73 & [75] & 74 & N117 & 30 & 90.8 & 84.8 & 77 & [76] \\
\hline 36 & N115 & 100 & 93 & 55 & 51 & [75] & 75 & N117 & 60 & 86.3 & 80.6 & 69.6 & [77] \\
\hline 37 & N115 & 20 & 93 & 87 & 81 & [78] & 76 & N117 & 50 & 93 & 82.3 & 77 & [79] \\
\hline 37 & N115 & 100 & 97 & 62 & 61 & [78] & 77 & N117 & 60 & 92.8 & 79.6 & 73.8 & [80] \\
\hline 38 & N115 & 80 & 94.6 & 82.1 & 86.8 & [81] & 78 & N117 & 40 & 90 & 92 & 83 & [82] \\
\hline 39 & N115 & 60 & 91.7 & 92.3 & 84.7 & [83] & 78 & N117 & 200 & 95 & 66 & 63 & [82] \\
\hline
\end{tabular}


Table 3. Cont.

\begin{tabular}{|c|c|c|c|c|c|c|c|c|c|c|c|c|c|}
\hline \multirow{2}{*}{ MS } & \multirow{2}{*}{ Membrane } & \multirow{2}{*}{$\frac{\mathrm{CD}}{\mathrm{mA} \mathrm{cm^{-2 }}}$} & \multirow{2}{*}{$\begin{array}{c}\mathrm{CE} \\
\%\end{array}$} & \multirow{2}{*}{$\begin{array}{l}\text { VE } \\
\%\end{array}$} & \multirow{2}{*}{$\begin{array}{c}\text { EE } \\
\%\end{array}$} & \multirow{2}{*}{ Ref. } & \multirow{2}{*}{ MS } & \multirow{2}{*}{ Membrane } & \multirow{2}{*}{$\frac{\mathrm{CD}}{\mathrm{mA} \mathrm{cm}^{-2}}$} & \multirow{2}{*}{$\begin{array}{c}\mathrm{CE} \\
\%\end{array}$} & \multirow{2}{*}{$\begin{array}{l}\text { VE } \\
\%\end{array}$} & \multirow{2}{*}{$\begin{array}{c}\mathrm{EE} \\
\%\end{array}$} & \multirow{2}{*}{ Ref. } \\
\hline & & & & & & & & & & & & & \\
\hline 40 & N115 & 80 & - & - & 84.5 & {$[46]$} & 79 & N117 & 80 & 92 & 87 & 80 & [84] \\
\hline 40 & N115 & 160 & - & - & 75.2 & [46] & 80 & N117 & 20 & 82 & 90 & 74 & [85] \\
\hline 41 & N115 & 40 & 98 & 72 & 70 & [86] & 80 & N117 & 80 & 92 & 71 & 65 & [85] \\
\hline 42 & N115 & 80 & 96.64 & 86 & 83 & [32] & 81 & N117 & 10 & 72.5 & 97.5 & 71 & [87] \\
\hline 43 & N115 & 80 & 94 & 87 & 81.8 & {$[88]$} & 81 & N117 & 80 & 92.5 & 84 & 78 & [87] \\
\hline 44 & N115 & 50 & 97 & 89 & 86.3 & [89] & 82 & N117 & 40 & 89 & 91 & 81 & [90] \\
\hline 44 & N115 & 150 & 98 & 70 & 68.6 & [89] & 82 & N117 & 200 & 94 & 67 & 63 & [90] \\
\hline 45 & N115 & 50 & 96 & 89 & 86 & [91] & 83 & N117 & 40 & 90 & 92 & 83 & [92] \\
\hline 46 & N115 & 40 & 93 & 93 & 86.5 & [93] & 83 & N117 & 200 & 95 & 67 & 63.7 & [92] \\
\hline 46 & N115 & 120 & 96 & 80 & 76.8 & [93] & 84 & N117 & 40 & 95.6 & 91 & 86.9 & [94] \\
\hline 47 & N115 & 40 & 96.26 & 94.3 & 90.77 & [95] & 85 & N117 & 40 & 94 & 91 & 86 & [96] \\
\hline 47 & N115 & 160 & 98.11 & 79.98 & 78.47 & [95] & 85 & N117 & 100 & 96 & 80 & 76.8 & [96] \\
\hline 48 & N115 & 80 & 93 & 88 & 82 & [97] & 86 & N117 & 40 & 95 & 90 & 85.5 & [98] \\
\hline 49 & N115 & 40 & 91 & 93 & 85 & [99] & 86 & N117 & 140 & 96 & 77 & 74 & [98] \\
\hline 49 & N115 & 160 & 94 & 78 & 73 & [99] & 87 & N117 & 20 & 94 & 95 & 89.3 & {$[100]$} \\
\hline 50 & N115 & 50 & 91.3 & 91.9 & 84 & [101] & 87 & N117 & 80 & 96 & 83 & 79.7 & {$[100]$} \\
\hline 51 & N115 & 80 & 92 & 88 & 82 & [102] & 88 & N117 & 40 & 95.9 & 89.7 & 86 & [103] \\
\hline 13 & N115 & 80 & 95 & 90 & 86 & [27] & 89 & N117 & 20 & 81 & 87 & 72 & [104] \\
\hline \multirow[t]{2}{*}{13} & N115 & 320 & 97 & 67 & 65 & [27] & 89 & N117 & 80 & 94 & 67 & 65 & [104] \\
\hline & & & & & & & 90 & N117 & 20 & 86.2 & 90.3 & 77.8 & [105] \\
\hline
\end{tabular}

Figure $2 \mathrm{~b}$ shows the energy efficiency achieved using Nafion membranes at current densities below $100 \mathrm{~mA} \mathrm{~cm}{ }^{-2}$. The differing VRFB cells and varied test conditions lead to the specific results for each membrane used at the respective current density. For example, energy efficiencies between $66 \%$ and $89 \%$ could be achieved for the current density of $80 \mathrm{~mA} \mathrm{~cm}^{-2}$ using N212 membranes, between $61 \%$ and $87 \%$ for N115 membranes and between $65 \%$ and $80 \%$ for N117 membranes. At current densities between 20 and $40 \mathrm{~mA} \mathrm{~cm}^{-2}$, efficiencies of over $90 \%$ were measured with N212 and N115.

Figure $2 \mathrm{c}$ shows the energy efficiencies at current densities of at least $100 \mathrm{~mA} \mathrm{~cm}{ }^{-2}$ for N212, N115 and N117. While with current densities below $100 \mathrm{~mA} \mathrm{~cm}^{-2}$ there is no correlation between current density and energy efficiency, with current densities of at least $100 \mathrm{~mA} \mathrm{~cm}^{-2}$ there is a tendency of decreasing efficiency with increasing current density up to above $200 \mathrm{~mA} \mathrm{~cm}^{-2}$ for all three membranes N212, N115 and N117. Furthermore, the highest energy efficiencies when using the respective membrane at 100 and $200 \mathrm{~mA} \mathrm{~cm}^{-2}$ show the increase in efficiency when the membrane thickness decreases:

$$
\mathrm{EE}(\mathrm{N} 212)>\mathrm{EE}(\mathrm{N} 115)>\mathrm{EE}(\mathrm{N} 117) .
$$

Figure 2 clearly shows that the energy efficiency of VRFB cells is related to the membrane used. However, differences in efficiency cannot be exclusively allocated to the specific membrane used due to different cell designs and operation modes of the cells.

\section{Polymer Membrane Development}

Synthetic membranes are used in various processes such as reverse osmosis, water filtration, dialysis or electrolysis. The respective separation requires membranes with a 
certain structure and certain chemical properties which result from the specific manufacturing process, formulation conditions and materials used. In electrochemical cells, electrical properties of membranes (membrane/electrolyte) and selectivity are particularly important. VRFB research focuses mainly on polymer membranes due to the generally low material and manufacturing costs involved when produced in large quantities.

\subsection{Membrane Structures}

Membrane properties not only depend on materials chosen but also on the manufacturing process. The general classification according to their structure and material is shown in Figure 3 [106-108]. Whereas dense membranes can be obtained by polymer extrusion or phase inversion by solvent evaporation (e.g., doctor blading) [106,109], porous membranes and separators are made by "stretching" semicrystalline polymer films [106,108]. Other known processes for the production of porous membranes are the sintering of polymer powders, thermal-induced phase separation or diffusion-induced phase separation. Ion-exchange membranes, which are a type of dense membrane, are produced by phase inversion, by solvent evaporation or special polymerization processes with corresponding chemical post-treatment [110].

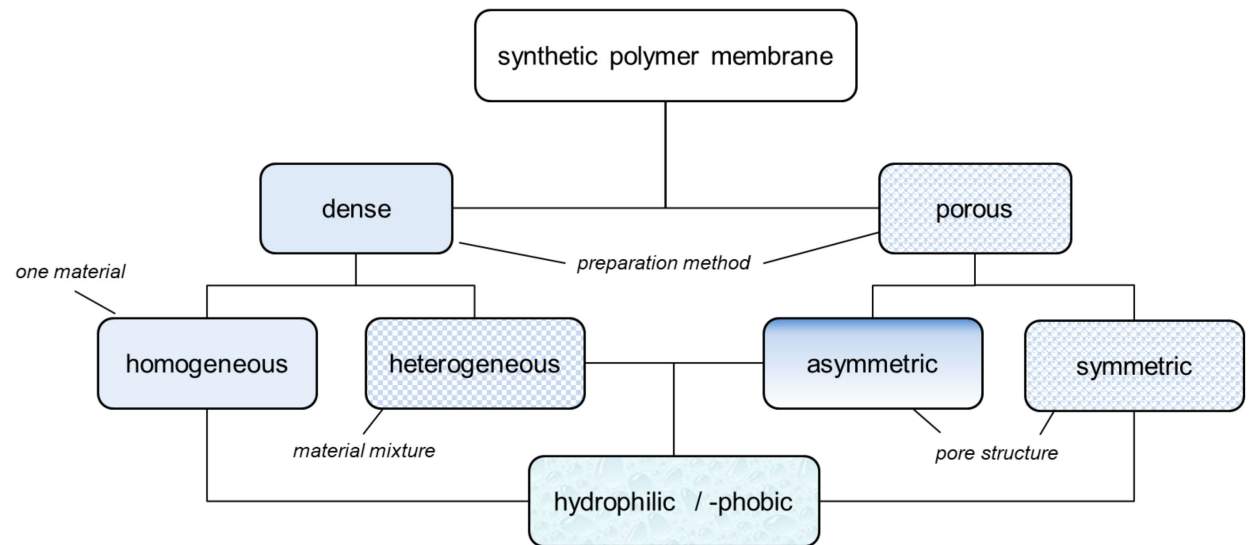

Figure 3. Classification of synthetic membranes according to their structure.

While several materials are required to prepare a heterogeneous dense membrane, e.g., composite or multilayer membrane, only one polymer or a polymer blend is typically used for homogeneous dense and symmetrically porous membranes. Asymmetrically porous membranes can be prepared from one (integral-asymmetrical) or several polymers (composite-asymmetrical) [107]. In the production of integrally asymmetrically porous membranes, first a thin skin is created on a polymer solution by phase inversion through solvent evaporation. Following this, the remaining solvent is extracted from the polymer solution by immersion in a precipitation bath. With asymmetrical composite membranes, a thin layer (dense or reduced porosity) is applied to a symmetrically porous membrane [107].

Ion-exchange membranes such as Nafion and symmetrically porous membranes such as Daramic have been tested in VRFB for many years. The chemical modification of Daramic by applying charge carriers has resulted in an improvement of the energy efficiency [111]. In this review the membrane structures of published membrane samples are divided into homogeneous dense (dho), heterogeneous dense (dhe), symmetrically porous (sym) and asymmetrically porous (asym) membranes.

\subsection{Membrane Polymers}

In this review, modified polymer membranes for VRFB are presented from more than 190 publications up to and including the year 2020. In most cases, the developments are based on polymers that can already be produced on a large scale. These include poly(ether ether ketone) (PEEK), poly(sulfone) (PSU), poly(ether sulfone) (PES), poly(phenyl sulfone) (PPSU), poly(vinylidene fluoride) (PVDF), poly(ethylene-tetrafluoroethylene) (ETFE), 
poly(benzimidazole) (PBI), poly(imide) (PI), perfluorosulfonic acid (PFSA), poly(phenylene ether) (PPE) and poly(tetrafluoroethylene) (PTFE). Other modified membrane polymers investigated include fluorinated poly(arylene ether) (FPAE), poly(fluorenyl ether ketone) (PF), poly(phenylene) (PPh) and poly(phthalazinone ether ketone) (PPEK).

For a clear presentation of the membrane developments, these are summarized according to the most important structural feature (C-F, ether-ketones, ether-sulfones, fluorenyles, phenylenes, benzimidazoles, phthalazinone-ether and imides) of the monomer (Table 4).

Table 4. Polymers for the preparation of VRFB membranes.

\begin{tabular}{|c|c|c|}
\hline Polymer & Group & Structure Examples \\
\hline PFSA, PTFE, PVDF, ETFE & fluoro-carbons & $-\mathrm{C}-\mathrm{F}$ \\
\hline Poly (phenylene) & hydro-carbon & \\
\hline Poly (ether ketone) & hydro-carbon & \\
\hline Poly (ether sulfone) & hydro-carbon & \\
\hline Poly (fluorenyl ether) & hydro-carbon & \\
\hline Poly (phenylene ether) & hydro-carbon & \\
\hline other & hydro-carbon & - \\
\hline Poly (benzimidazole) & $N$-heterocycles & \\
\hline Poly (phthalazinone ether ketone) & $N$-heterocycles & \\
\hline Poly (imide) & $\mathrm{N}$-heterocycles & \\
\hline
\end{tabular}

Cation-exchange membranes (CEM), anion-exchange membranes (AEM) and amphoteric ion-exchange membranes (AIEM) are based on polymers with covalently bonded charges. AEM* and AIEM* are based on neutral polymers which build up a positive charge through interaction with hydronium-ions by lowering the $\mathrm{pH}$ value in their environment below $\mathrm{pH} 7$. 


\section{Membrane Developments in Recent Years}

The number of publications concerning membrane samples for VRFB has increased significantly since 2012. Polymer membranes can be subdivided with regard to elementary components of the polymer main chains. A distinction is made between fluorine-based polymers (fluoro-carbon) and fluorine-free (hydro-carbon) polymers. Nitrogen containing heterocycles-based polymers ( $N$-heterocycles) are also used.

For membranes composed of polymer mixtures, this subdivision refers to the polymer with the larger molar or mass fraction in the mixture. Polymers from these three groups are used in the development of polymer membranes for VRFB. In order to optimize the performance or the costs of a VRFB cell, suitable methods are selected to make chemical modifications to the polymers or to generate desired spatial structures. These chemical modifications can be applied to commercially available films (Figure 4a, MS107), commercial polymers (Figure $4 b$, MS151), or proprietary polymer syntheses (Figure $4 b$, MS143). The chemical methods aim to generate acidic, basic or amphoteric polymers whose chemical function influences the internal resistance of the VRFB and the cross-over between the half cells. As shown in Figure 3, synthetic membranes with distinctive structures can be used in VRFB cells.

The developed and published membrane samples of the past years show these different structures and were tested in VRFB. An exception is the dense hydrophobic membrane, which can be used as a separation medium for gases but would act as an insulator in a VRFB. Dense homogeneous membranes are composed of fluoro-carbons (e.g., PFSA, ETFEg-X, PVDF-g-X, sFPAE), hydro-carbons (e.g., QDAPP, sPEEK, sPSU) and N-heterocycles (PBI, sPI).

Dense heterogeneous membranes represent a very frequently used membrane type, because it is relatively easy to influence their properties such as porosity (Figure 5b, MS189) by adding disperse components. Dense heterogeneous membranes can be found in fluorocarbon, hydro-carbon and $\mathrm{N}$-heterocycle-based membranes.

Porous membranes can either have hydrophilic or hydrophobic character. Symmetrically porous (Figure 4c, MS266, hydrophilic) and asymmetrically porous (Figure 4a, MS98, hydrophobic) membranes get prepared and tested in scientific workgroups.

Furthermore, multi-layer membranes are constructed to achieve certain properties, such as improved chemical stability (Table 7, MS167), lower costs (Figure 4b, MS147) or lower cross-over (Figure 4a, MS95).

Figures 4 and 5 show the observed energy efficiency of VRFB cells using the developed membrane samples and the publication year. Figure 4 shows results obtained with current densities less than $100 \mathrm{~mA} \mathrm{~cm}^{-2}$ and Figure 5 shows results obtained with current densities greater than $100 \mathrm{~mA} \mathrm{~cm}{ }^{-2}$. The figures provide an overall picture of the achievable energy efficiencies of VRFB with different flow-battery targeted membranes.

When comparing the results of the commercial membranes in Figure $2 b$ with the results in Figure 4, it can be observed that VRFB with new membrane developments often achieve higher energy efficiencies than VRFB with each reference membranes. 

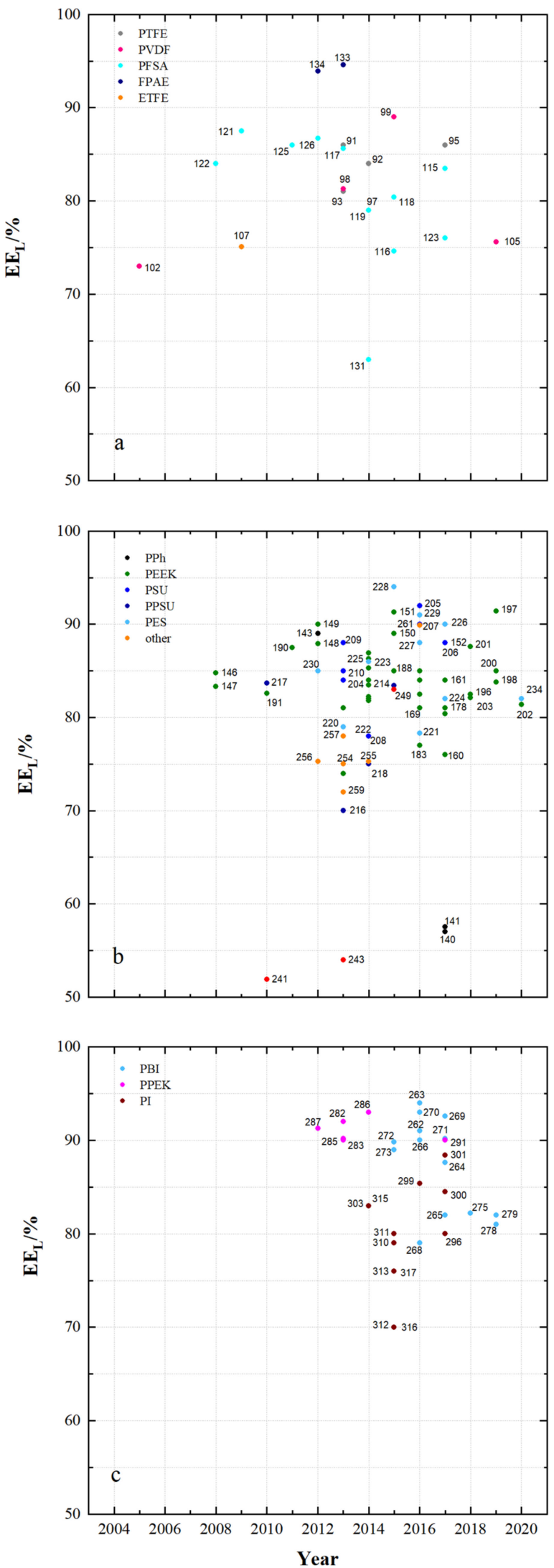

Figure 4. The energy efficiency of VRFB cells at current densities $<100 \mathrm{~mA} \mathrm{~cm}^{-2}$ using: (a) fluorocarbons, (b) hydro-carbons and (c) $N$-heterocycles. 

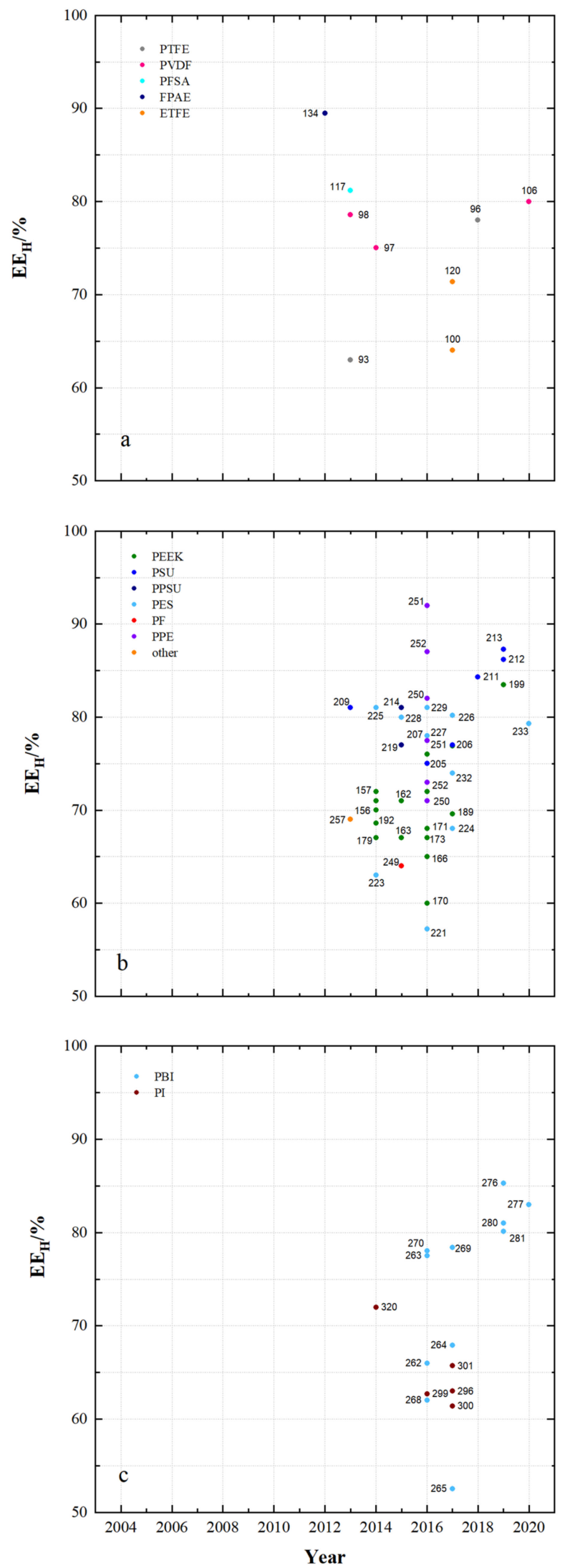

Figure 5. The energy efficiency of VRFB cells by using the developed membrane sample at current densities $\geq 100 \mathrm{~mA} \mathrm{~cm}^{-2}$ in recent years: (a) fluoro-carbons, (b) hydro-carbons and (c) $N$-heterocycles. 
A direct comparison of membranes is only possible in the same cell and under identical experimental conditions. The comparison can be expressed in numerical terms by comparing the energy efficiencies achieved according to Equation (1). A value less than 1 is obtained if the energy efficiency of the VRFB using flow-battery targeted membranes is less than the energy efficiency of the VRFB with a reference membrane. $\mathrm{EE}_{\mathrm{r}}$ (energy efficiency ratio) is larger than one if the energy efficiency of the VRFB using flow-battery targeted membranes is higher. It should be mentioned that modifications to the cell other than the membrane further influence individual $\mathrm{EE}$ and thus the resulting ratio $\mathrm{EE}_{\mathrm{r}}$.

$$
\mathrm{EE}_{\mathrm{r}}=\frac{\mathrm{EE}_{1}}{\mathrm{EE}_{2}}
$$

$\mathrm{EE}_{1}$ is the energy efficiency of the VRFB cell using flow-battery targeted membranes and $\mathrm{EE}_{2}$ the energy efficiency of the VRFB cell using a reference membrane. These energy efficiency ratios as well as the reference membranes are also given in Tables 5-14.

While energy efficiency describes the performance of a VRFB by charge and discharge cycles, membrane characteristics are usually influenced by a number of parameters. The frequently investigated membrane properties which can influence VRFB performance are the membrane thickness, water uptake, ion-exchange capacity, electrical resistance and the diffusion coefficient for vanadyl-cations. Furthermore, the ion-selectivity [112] is an important quality. Equation (2), like Equation (1), calculates a ratio for comparison to the reference membrane.

$$
\mathrm{D}_{\mathrm{r}}=\frac{\mathrm{D}_{\mathrm{c} 1}}{\mathrm{D}_{\mathrm{c} 2}}
$$

$\mathrm{D}_{\mathrm{c} 1}$ is the diffusion coefficient $\left(\mathrm{VO}^{2+}\right)$ of the membrane samples and $\mathrm{D}_{\mathrm{c} 2}$ the diffusion coefficient $\left(\mathrm{VO}^{2+}\right)$ of the reference membranes.

Tables 5-14 list the best membrane sample selected from each publication. The tables contain information about the specific membrane sample as well as VRFB performance data. In addition to the sample name from the respective publication, the starting polymer, the structure and the chemical character (AEM, CEM or AIEM) are also listed.

\subsection{Membrane Properties}

Membrane samples are produced by chemical modification of commercially available membranes and films or by coating and subsequent phase inversion (solvent evaporation or precipitation). The thickness of the membrane samples depicted in Figure 6 is the result from these processes and also of possibly occurring membrane swelling in battery electrolyte.

Generally, the thickness of the membrane defines the distance between the electrodes. For a low electric resistance of an electrochemical cell, the smallest distance between the electrodes is desirable.

The fluoro-carbon-based membranes (Figure 6a) have thicknesses from $25 \mu \mathrm{m}$ to $225 \mu \mathrm{m}$, the hydro-carbon-based membranes in Figure $6 \mathrm{~b}$ thicknesses from $35 \mu \mathrm{m}$ to $390 \mu \mathrm{m}$ and the $N$-heterocycle-based membranes in Figure $6 \mathrm{c}$ thicknesses from $15 \mu \mathrm{m}$ to $260 \mu \mathrm{m}$.

The PFSA membranes MS116, MS120, MS121, MS124, MS126, MS127 and MS128 are modified Nafion (N117) membranes with an original thickness of about $180 \mu \mathrm{m}$.

ETFE membranes modified by grafting (MS107-MS114) use commercially available ETFE films $(25$ or $50 \mu \mathrm{m})$. A commercial film is also used for the pore-filled PTFE membrane (MS92).

The non-ionic PVDF membranes MS97, MS98 and MS99 have porous structures. The hydrophobic and asymmetrically porous MS98 has a separation layer in the submicron range with pore sizes of about $50 \mathrm{~nm}$. The hydrophilic asymmetrically porous MS99 has a similar structure. 

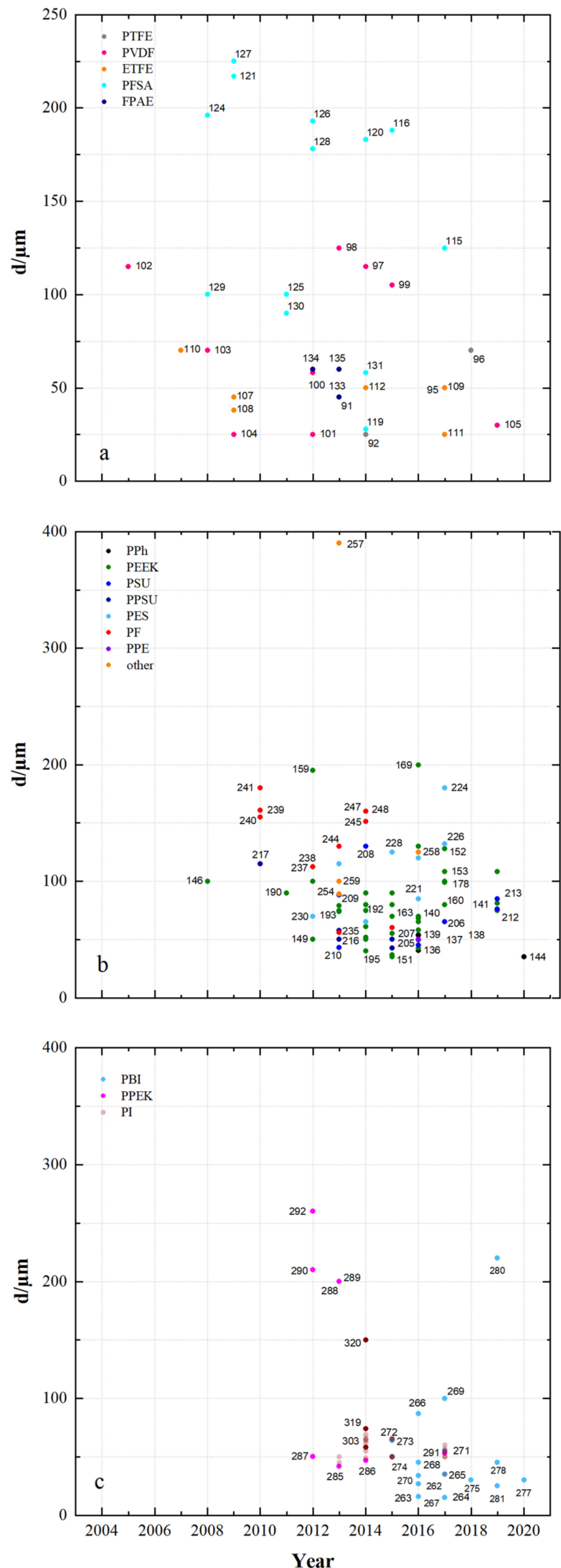

Figure 6. The thickness of developed membranes in recent years: (a) fluoro-carbons, (b) hydrocarbons and (c) $N$-heterocycles. 
Further asymmetric membranes can be found in the group of hydro-carbon-based polymer membranes. These include MS208 with an average pore size of $1.78 \mathrm{~nm}$, MS225 (asymmetrically composed) with a Nafion separating layer of approximately $1 \mu \mathrm{m}$ and MS229 (asymmetrically composed) modified by a zeolite layer with pore sizes of $0.3-1 \mathrm{~nm}$.

In addition to an asymmetrically porous membrane, MS269 with a separating layer of about $5 \mu \mathrm{m}$, symmetrically porous PBI-based membranes with thicknesses between $34 \mu \mathrm{m}$ (MS270) and $87 \mu \mathrm{m}$ (MS266) were developed.

The thickness of a membrane defines the distance between the electrodes in the VRFB and directly influences the material cost. Furthermore, separation effect increases with the thickness of the membrane up to a critical pore size.

The simultaneous reactions on the anode and cathode surfaces during charging and discharging require the exchange of protons. Sulfuric acid, the solvent of the reactive vanadium species, as well as sulfonated polymers are excellent proton conductors. The ion exchange capacity of a membrane describes the acid concentration of the polymer membrane. The ion-exchange capacities ( IEC $_{c}$, cations) indicated for PFSA membranes are between 0.85 and $1.67 \mathrm{mmol} \mathrm{g}^{-1}$, whereby MS129 containing sulfonated PEEK in addition to Nafion enables the highest $\mathrm{IEC}_{\mathrm{c}}$. The FPAE membranes have an $\mathrm{IEC}_{\mathrm{C}}$ of 1.6 to $1.8 \mathrm{mmol} \mathrm{g}^{-1}$.

For the DAPP membranes in Figure $7 \mathrm{~b}$ the $\mathrm{IEC}_{\mathrm{c}}$ is between 1.4 and $1.8 \mathrm{mmol} \mathrm{g}^{-1}$ and for the PEEK membranes between 0.74 (MS193) and $2.43 \mathrm{mmol} \mathrm{g}^{-1}$ (MS165). MS206 has an IEC $_{\mathrm{c}}$ of $2.04 \mathrm{mmol} \mathrm{g}^{-1}$, MS217 to MS219 an IEC $_{\mathrm{c}}$ of 1.2 to $1.95 \mathrm{mmol} \mathrm{g}^{-1}$, MS230 an IEC $_{\mathrm{c}}$ of $0.7 \mathrm{mmol} \mathrm{g}^{-1}$ and MS231 an IEC $_{\mathrm{c}}$ of $2.07 \mathrm{mmol} \mathrm{g}^{-1}$. The PF-based membranes have an $\mathrm{IEC}_{\mathrm{C}}$ of $1.47 \mathrm{mmol} \mathrm{g}^{-1}$ to $1.96 \mathrm{mmol} \mathrm{g}^{-1}$. For MS259 and MS260 an IEC $\mathrm{C}$ of 1.2 and $0.69 \mathrm{mmol} \mathrm{g}^{-1}$ was measured.

The PBI membranes in Figure $7 \mathrm{c}$ have an $\mathrm{IEC}_{\mathrm{c}}$ of 0.24 to $1.56 \mathrm{mmol} \mathrm{g}^{-1}$, the PPEK membranes an IEC $_{\mathrm{c}}$ of 1.14 to $1.51 \mathrm{mmol} \mathrm{g}^{-1}$ and the PI membranes an IECc of 0.4 to $1.75 \mathrm{mmol} \mathrm{g}^{-1}$. In the development of membranes, IEC $_{\mathrm{c}}$ between 1 and $2 \mathrm{mmol} \mathrm{g}^{-1}$ is predominantly achieved. The PFSA membranes in the fluoro-carbon group increasingly exhibit IEC $_{\mathrm{C}}$ smaller than $1 \mathrm{mmol} \mathrm{g}^{-1}$. In the group of hydro-carbon-based membranes, especially when using PEEK, IEC $\mathrm{c}_{\mathrm{c}}$ of more than $2 \mathrm{mmol} \mathrm{g}^{-1}$ can be reached. Figure 8 shows the diffusion coefficients for vanadyl cations of membrane samples that have been tested and published for use in VRFB since 2005. Low diffusion coefficients lead to low vanadium crossover during charging and discharging of the battery and therefore influences the coulombic efficiency. Diffusion coefficients $\left(\mathrm{VO}^{2+}\right)$ from $2.9 \times 10^{-9}$ to $6.72 \times 10^{-6} \mathrm{~cm}^{2} \mathrm{~min}^{-1}$ for fluoro-carbon-based membranes and $1.6 \times 10^{-9}$ to $4.2 \times 10^{-6} \mathrm{~cm}^{2} \mathrm{~min}^{-1}$ for hydrocarbons, as well as $1.28 \times 10^{-11}$ to $2.6 \times 10^{-6} \mathrm{~cm}^{2} \mathrm{~min}^{-1}$ for $N$-heterocycles, have been published. Selected diffusion coefficients ranges:

- $\quad$ PTFE $4.62 \times 10^{-8}$ to $7.1 \times 10^{-7} \mathrm{~cm}^{2} \mathrm{~min}^{-1}$

- PVDF $6.7 \times 10^{-8}$ to $7.9 \times 10^{-7} \mathrm{~cm}^{2} \mathrm{~min}^{-1}$

- $\quad$ ETFE $2.9 \times 10^{-9}$ to $3.9 \times 10^{-8} \mathrm{~cm}^{2} \mathrm{~min}^{-1}$

- PFSA $3.6 \times 10^{-9}$ to $6.72 \times 10^{-6} \mathrm{~cm}^{2} \mathrm{~min}^{-1}$

- $\quad$ FPAE (MS134) $1.16 \times 10^{-8} \mathrm{~cm}^{2} \mathrm{~min}^{-1}$

- PPh $3.3 \times 10^{-9}$ to $1.4 \times 10^{-6} \mathrm{~cm}^{2} \mathrm{~min}^{-1}$

- PEEK $1.05 \times 10^{-9}$ to $4.2 \times 10^{-6} \mathrm{~cm}^{2} \mathrm{~min}^{-1}$

- PSU $1.5 \times 10^{-8}$ to $2.94 \times 10^{-6} \mathrm{~cm}^{2} \mathrm{~min}^{-1}$

- PPSU $1.6 \times 10^{-9}$ to $2.07 \times 10^{-7} \mathrm{~cm}^{2} \mathrm{~min}^{-1}$

- PES $1.41 \times 10^{-8}$ to $4 \times 10^{-6} \mathrm{~cm}^{2} \mathrm{~min}^{-1}$

- PF $8.8 \times 10^{-8}$ to $9.85 \times 10^{-7} \mathrm{~cm}^{2} \mathrm{~min}^{-1}$

- PPE $1.1 \times 10^{-8}$ to $3.6 \times 10^{-8} \mathrm{~cm}^{2} \mathrm{~min}^{-1}$

- Other $6.9 \times 10^{-8}$ to $1.56 \times 10^{-7} \mathrm{~cm}^{2} \mathrm{~min}^{-1}$

- PBI $1.28 \times 10^{-11}$ to $5.74 \times 10^{-7} \mathrm{~cm}^{2} \mathrm{~min}^{-1}$

- PPEK $1.24 \times 10^{-7}$ to $5.75 \times 10^{-7} \mathrm{~cm}^{2} \mathrm{~min}^{-1}$

- PI $4.8 \times 10^{-8}$ to $2.6 \times 10^{-6} \mathrm{~cm}^{2} \mathrm{~min}^{-1}$. 

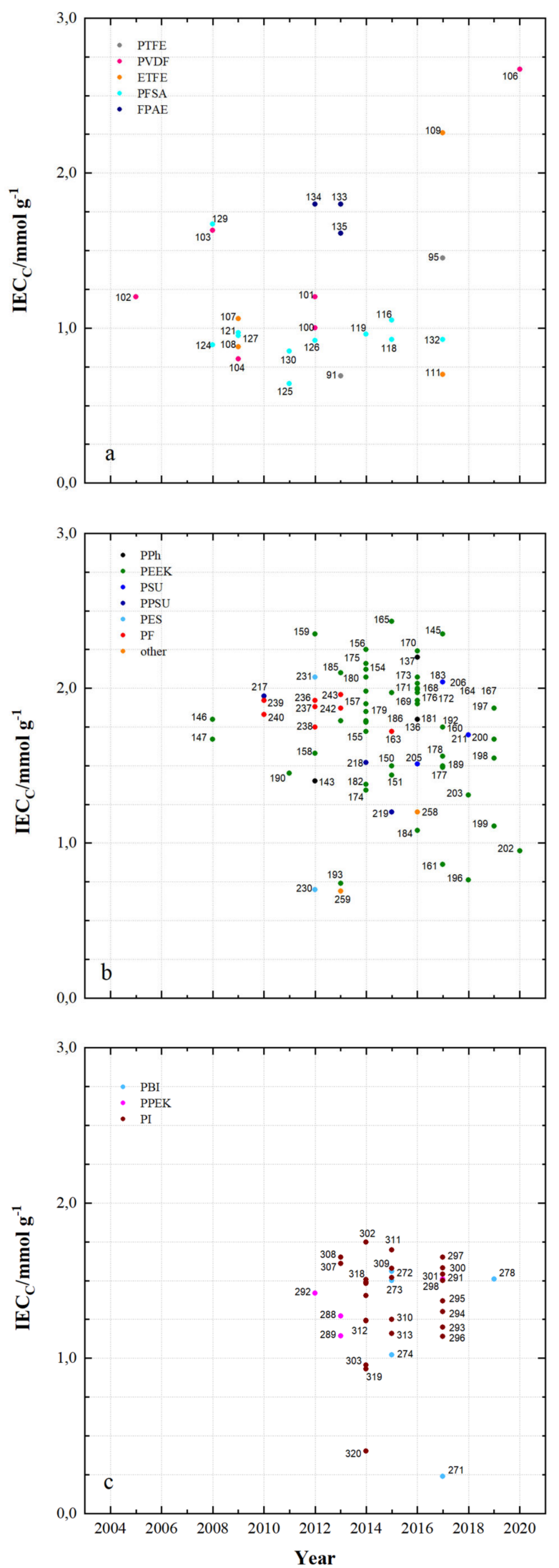

Figure 7. The ion-exchange capacity $\left(\mathrm{IEC}_{\mathrm{c}}\right.$ ) of tested polymer membranes in recent years: (a) fluorocarbons, (b) hydro-carbons and (c) $N$-heterocycles. 

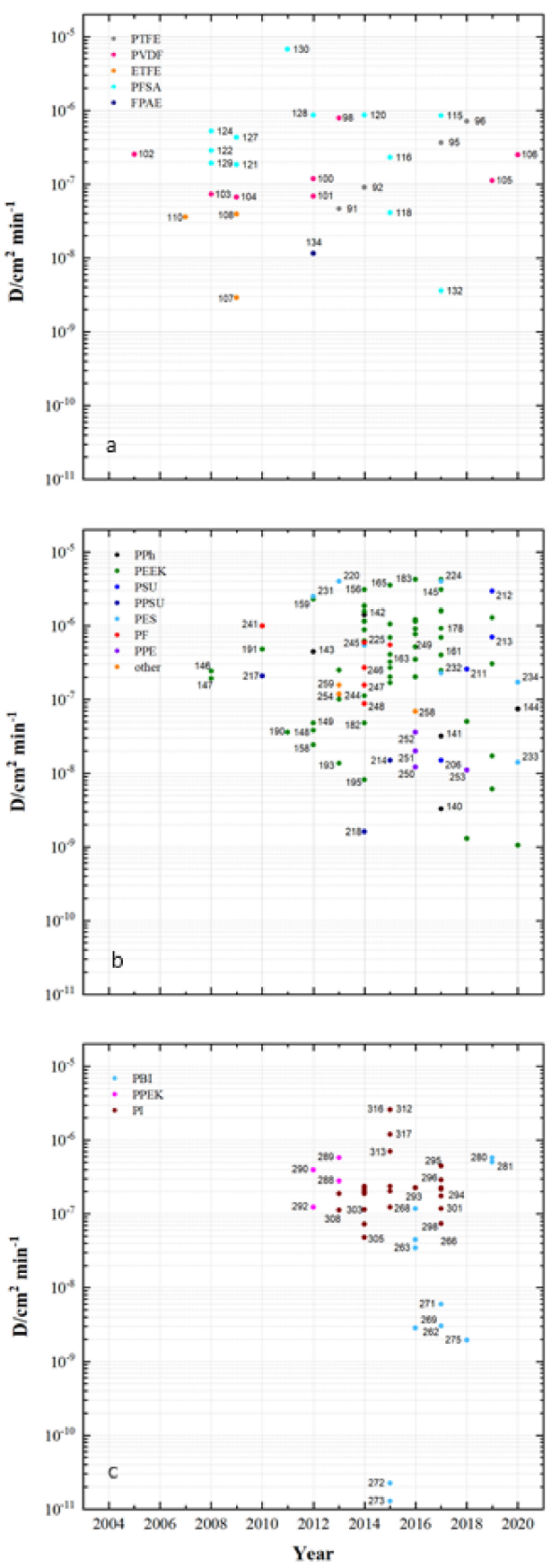

Figure 8. Measured diffusion coefficients of tested polymer membranes in recent years: (a) fluorocarbons, (b) hydro-carbons and (c) N-heterocycles.

The results on membrane thickness, ion-exchange capacity and vanadyl permeation summarized in Figures 6-8, as well as the Supplementary data showing water uptake and anion-exchange capacity of membrane samples, are the most frequently investigated characteristics in publications on membrane development for VRFB cells. Other important properties are electrical resistance and ion-selectivity, for which the goal is to achieve a high proton conductivity, combined with the lowest vanadium-ion permeability possible. It is described in [113] that membrane thickness in particular has an influence on this and can be optimized accordingly.

With increasing ion-exchange capacity, water uptake increases in non-crosslinked membranes $[84,114]$. 
Table 5. List of fluoro-carbon membrane samples.

\begin{tabular}{|c|c|c|c|c|c|c|c|c|c|c|c|c|c|c|c|c|}
\hline \multirow{3}{*}{ MS } & \multirow{3}{*}{$\begin{array}{c}\begin{array}{c}\text { Membrane } \\
\text { Sample }\end{array} \\
\begin{array}{c}\text { Polymer/Sample } \\
\text { Name }\end{array}\end{array}$} & \multicolumn{2}{|c|}{ Membrane } & \multicolumn{6}{|c|}{ Membrane Properties } & \multicolumn{5}{|c|}{ VRFB Properties } & \multicolumn{2}{|c|}{ Reference } \\
\hline & & \multirow{2}{*}{ Struc } & \multirow{2}{*}{ Chem } & $\mathrm{d}$ & IEC $_{\mathrm{C}}$ & $\mathrm{IEC}_{\mathrm{A}}$ & WU & $\mathrm{D}_{\mathrm{C}}$ & $\mathrm{D}_{\mathrm{r}}$ & $\mathrm{CD}$ & $\mathrm{CE}$ & VE & $\mathrm{EE}$ & $\mathrm{EE}_{\mathrm{r}}$ & \multirow{2}{*}{ Mem } & \multirow{2}{*}{ Pub } \\
\hline & & & & $\mu \mathrm{m}$ & $\mathrm{mmol} \mathrm{g}^{-1}$ & $\mathrm{mmol} \mathrm{g}^{-1}$ & wt. $\%$ & $\mathrm{~cm}^{2} \min ^{-1}$ & $\%$ & $\mathrm{~mA} \mathrm{~cm}^{-2}$ & $\%$ & $\%$ & $\%$ & & & \\
\hline 91 & PTFE/Nafion/P/N & dhe & CEM & 45 & 0.69 & - & 24.9 & $4.62 \times 10^{-8}$ & 0.7 & 80 & 96 & 80 & 76 & 1.070 & N212 & [53] \\
\hline 92 & PTFE/P/N/S-7 & dhe & CEM & 25 & - & - & 65.5 & $9 \times 10^{-8}$ & 0.45 & 80 & 94 & 87 & 82 & 1.155 & $\mathrm{P} / \mathrm{N}$ & [115] \\
\hline 93 & PTFE/SiO2 & sym & - & - & - & - & 48 & - & - & 50 & 93 & 86 & 80 & 0.930 & N115 & [91] \\
\hline 94 & PTFE/SPEEK/SP60 & dhe & CEM & - & - & - & 36 & - & - & 80 & 93 & 90 & 84 & 1.021 & N115 & [88] \\
\hline 96 & PTFE/SE3/P & dhe & CEM & 70 & - & - & 29.8 & $7.1 \times 10^{-7}$ & 0.178 & 100 & 99 & 79 & 78 & 1.147 & N117 & [116] \\
\hline 97 & PVDF/M7 & sym & - & 115 & - & - & - & - & - & 120 & 94 & 79 & 75 & - & - & [117] \\
\hline 98 & PVDF/M-23-125 & asym & - & 125 & - & - & - & $7.9 \times 10^{-7}$ & 0.664 & 80 & 95 & 83 & 79 & - & - & [118] \\
\hline 99 & PVDF/M2 & asym & - & 105 & - & - & - & - & - & 80 & 94 & 87 & 82 & 1.012 & N115 & [119] \\
\hline 100 & $\begin{array}{l}\text { PVDF-g-St-co- } \\
\text { / AIEM }\end{array}$ & dho & AIEM & 58 & 1 & - & - & $1.18 \times 10^{-7}$ & 0.153 & - & - & - & - & - & N117 & [120] \\
\hline 101 & PVDF g/AIEM & dho & AIEM & 25 & 1.2 & - & 48 & $6.9 \times 10^{-8}$ & 0.087 & - & - & - & - & - & N117 & [121] \\
\hline 102 & $\begin{array}{l}\text { PVDF-g- } \\
\text { PSSA/22 }\end{array}$ & dho & CEM & 115 & 1.2 & - & 26.4 & $2.53 \times 10^{-7}$ & 0.084 & 60 & 91 & 78 & 73 & 1.082 & N117 & [68] \\
\hline 104 & $\begin{array}{l}\text { PVDF-g-St-co- } \\
\text { / AIEM }\end{array}$ & dho & AIEM & 25 & 0.8 & 0.7 & 36 & $6.7 \times 10^{-8}$ & 0.084 & - & - & - & - & - & N117 & [123] \\
\hline 105 & $\begin{array}{l}\text { PVDF/SiO2- } \\
\text { SO3H_42 }\end{array}$ & dhe & CEM & 30 & - & - & 52.1 & $1.12 \times 10^{-7}$ & 0.108 & 60 & 90.3 & 83.5 & 75.6 & 0.967 & N115 & [124] \\
\hline 106 & PVDF/HA-45 & dho & CEM & - & 2.67 & - & 46 & $2.5 \times 10^{-7}$ & - & 100 & 95 & 84 & 80 & 1.08 & N117 & [125] \\
\hline 107 & $\begin{array}{c}\text { ETFE-g-PSSA-c- } \\
\text { AIEM-II }\end{array}$ & dho & AIEM & 45 & 1.06 & 1.24 & 36.1 & $2.9 \times 10^{-9}$ & 0.004 & 40 & 96 & 79 & 75 & 1.034 & N117 & [126] \\
\hline 108 & ETFE-g-PSSA & dho & CEM & 38 & 0.88 & - & 14.7 & $3.9 \times 10^{-8}$ & 0.057 & - & - & - & - & - & N117 & [126] \\
\hline 109 & $\begin{array}{l}\text { ETFE-g-GMA- } \\
\text { DG225 }\end{array}$ & dho & CEM & 50 & 2.4 & - & 181 & - & - & 100 & 87 & 73 & 64 & 1.067 & N117 & [56] \\
\hline 110 & $\begin{array}{c}\text { ETFE-g- } \\
\text { PDMAEMA/40\% }\end{array}$ & dho & AEM & 70 & - & 1.7 & 20 & $3.6 \times 10^{-8}$ & 0.042 & - & - & - & - & - & N117 & [127] \\
\hline 111 & ETFE-g-poly(VP) & dho & AIEM & 25 & 0.7 & - & - & - & - & 120 & 98 & 73 & 71 & 1.044 & N117 & [128] \\
\hline 112 & $\begin{array}{l}\text { ETFE-VB- } \\
\text { DABACO }\end{array}$ & dho & AEM & 50 & - & 1.55 & 38 & - & - & - & - & - & - & - & N115 & [129] \\
\hline 113 & ETFE-VB-DMA & dho & AEM & 50 & - & 1.33 & 8.8 & - & - & - & - & - & - & - & N115 & [129] \\
\hline
\end{tabular}


Table 5. Cont.

\begin{tabular}{|c|c|c|c|c|c|c|c|c|c|c|c|c|c|c|c|c|}
\hline \multirow{3}{*}{ MS } & \multirow{3}{*}{$\begin{array}{c}\begin{array}{c}\text { Membrane } \\
\text { Sample }\end{array} \\
\begin{array}{c}\text { Polymer/Sample } \\
\text { Name }\end{array} \\
\end{array}$} & \multicolumn{2}{|c|}{ Membrane } & \multicolumn{6}{|c|}{ Membrane Properties } & \multicolumn{5}{|c|}{ VRFB Properties } & \multicolumn{2}{|c|}{ Reference } \\
\hline & & \multirow{2}{*}{ Struc } & \multirow{2}{*}{ Chem } & d & IEC $_{C}$ & IEC $_{\mathrm{A}}$ & WU & $\mathrm{D}_{\mathrm{C}}$ & $\mathrm{D}_{\mathrm{r}}$ & $\mathrm{CD}$ & $\mathrm{CE}$ & VE & EE & $\mathrm{EE}_{\mathrm{r}}$ & \multirow{2}{*}{ Mem } & \multirow{2}{*}{ Pub } \\
\hline & & & & $\mu \mathrm{m}$ & $\mathrm{mmol} \mathrm{g}^{-1}$ & $\mathrm{mmol} \mathrm{g}^{-1}$ & wt. $\%$ & $\mathrm{~cm}^{2} \min ^{-1}$ & $\%$ & $\mathrm{~mA} \mathrm{~cm}^{-2}$ & $\%$ & $\%$ & $\%$ & & & \\
\hline 114 & ETFE-VB-TMA & dho & AEM & 50 & - & 1.64 & 38 & - & - & - & - & - & - & - & N115 & {$[129]$} \\
\hline 115 & PFSA AATMS & dhe & AIEM $^{*}$ & 125 & - & - & & $8.5 \times 10^{-7}$ & 0.218 & 80 & 96 & 88 & 83 & 1.051 & N115 & [71] \\
\hline 116 & $\begin{array}{l}\text { PFSA AATMS } \\
\text { (a-SiO2) }\end{array}$ & dhe & $\mathrm{AIEM}^{*}$ & 188 & 1.05 & - & 35.1 & $2.32 \times 10^{-7}$ & 0.268 & 80 & 91 & 72 & 69 & 1.062 & N117 & [64] \\
\hline 118 & $\begin{array}{l}\text { PFSA FC } \\
(\mathrm{N} / \mathrm{FC}-5)\end{array}$ & dhe & CEM & - & 0.925 & - & 31 & $4.1 \times 10^{-8}$ & 0.5 & 80 & 95 & 78 & 74 & 1.250 & N117 & {$[112]$} \\
\hline 119 & $\begin{array}{l}\text { PFSA GO } \\
(\text { GO-0.01) }\end{array}$ & dhe & CEM & 27.75 & 0.96 & - & 22.41 & - & - & 80 & 92 & 86 & 79 & 1.068 & N117 & [51] \\
\hline 120 & $\begin{array}{l}\text { PFSA ND } \\
\text { (AMH-3) }\end{array}$ & dhe & CEM & 183 & - & - & - & $8.64 \times 10^{-7}$ & 0.281 & 40 & 97 & 85 & 82 & 0.965 & N117 & [47] \\
\hline 121 & $\begin{array}{l}\text { PFSA Ormosil } \\
(\mathrm{N} / \mathrm{O})\end{array}$ & dhe & CEM & 217 & 0.97 & - & 23.6 & $1.85 \times 10^{-7}$ & 0.050 & 80 & 96 & 84 & 81 & 1.210 & N117 & {$[131]$} \\
\hline 122 & $\begin{array}{c}\text { PFSA [PDDA } \\
\text { PSS] } 5\end{array}$ & dhe & AIEM & - & - & - & - & $2.85 \times 10^{-7}$ & 0.095 & 80 & 98 & 73 & 72 & 1.091 & N117 & [85] \\
\hline 123 & $\begin{array}{c}\text { PFSA [PDDA } \\
\text { ZrP]3 }\end{array}$ & dhe & AIEM & - & - & - & 8 & - & - & 50 & 95 & 85 & 81 & 1.052 & N115 & {$[132]$} \\
\hline 124 & $\begin{array}{l}\text { PFSA PEI } \\
\text { (N/P2.5) }\end{array}$ & dhe & AIEM $^{*}$ & 196 & 0.89 & & & $5.23 \times 10^{-7}$ & & 50 & 96.2 & 88.4 & 85.1 & 1.001 & N117 & [67] \\
\hline 125 & $\begin{array}{l}\text { PFSA PVDF } \\
(\mathrm{N} / \mathrm{P} 0.2)\end{array}$ & dhe & CEM & 100 & 0.64 & - & 16.2 & - & - & 80 & 94 & 88 & 84 & 1.037 & $\mathrm{D}-520$ & {$[133]$} \\
\hline 126 & PFSA (N-sDDS) & dhe & CEM & 193 & 0.92 & - & 14.3 & - & - & 70 & 96 & 85 & 81 & 1.177 & N117 & {$[134]$} \\
\hline 127 & PFSA (N/Si/Ti) & dhe & CEM & 225 & 0.95 & - & 22.5 & $4.3 \times 10^{-7}$ & - & 30 & 95 & 82 & 78 & 1.012 & N117 & [76] \\
\hline 128 & PFSA (N-SiO2) & dhe & CEM & 178 & - & - & - & $8.64 \times 10^{-7}$ & - & 50 & 93 & 91 & 84 & 1.000 & N117 & {$[135]$} \\
\hline 129 & $\begin{array}{c}\text { PFSA SPEEK } \\
(\mathrm{N} / \mathrm{S})\end{array}$ & dhe & CEM & 100 & 1.67 & - & - & $1.93 \times 10^{-7}$ & 0.053 & 50 & 98 & 85 & 83 & 0.980 & N117 & {$[136]$} \\
\hline 130 & PFSA (N-TiO2) & dhe & CEM & 90 & 0.85 & - & 19.13 & $6.72 \times 10^{-6}$ & 0.297 & 60 & 89 & 81 & 72 & 1.027 & N117 & [77] \\
\hline 131 & PFSA (CS/PWA) & dhe & AIEM $^{*}$ & 58 & - & - & - & - & - & 60 & 82 & 93 & 76 & 1.070 & N212 & [44] \\
\hline 132 & PFSA (ZrNT) & dhe & CEM & 155 & 0.927 & - & - & $3.6 \times 10^{-9}$ & 0.010 & 40 & 98 & 81 & 79 & 1.090 & N117 & [137] \\
\hline 133 & SFPAE 1.8_45 & dho & CEM & 45 & 1.8 & - & - & - & - & 80 & 99 & 83 & 82 & - & - & {$[113]$} \\
\hline 134 & SFPAE 1.8 & dho & CEM & 60 & 1.8 & - & 48 & $1.16 \times 10^{-8}$ & 0.219 & 100 & 98 & 92 & 90 & 1.047 & N212 & [39] \\
\hline 135 & $\begin{array}{c}\text { SFPAE } \\
\text { (PVDF-co- } / 10 \%)\end{array}$ & dhe & CEM & 60 & 1.6 & - & 35 & - & - & 100 & 99 & 88 & 88 & 0.999 & SFPAE & {$[138]$} \\
\hline
\end{tabular}


Table 6. List of poly(phenylene)-based hydro-carbon membrane samples.

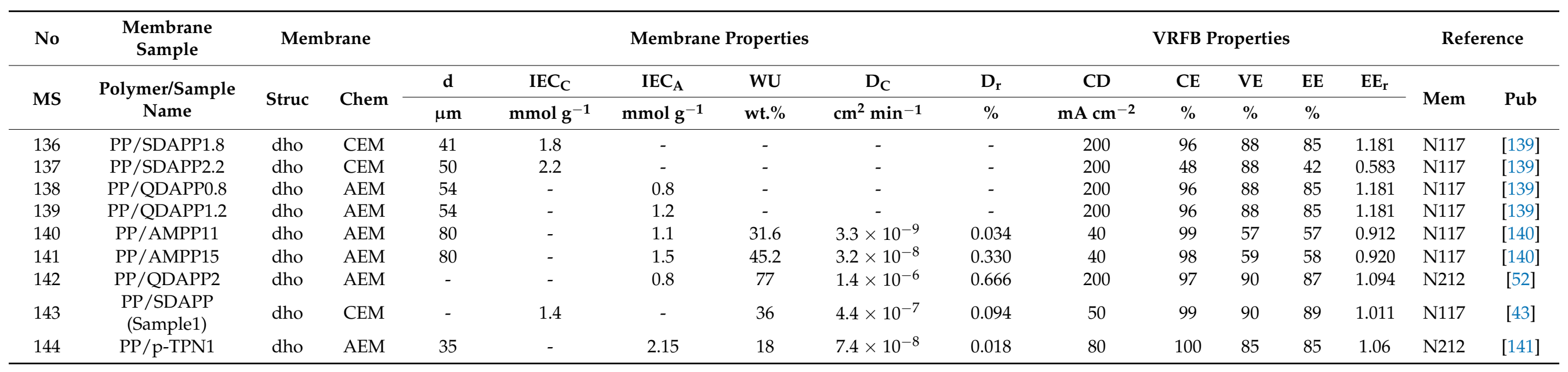

Table 7. List of ether-ketone-based hydro-carbon membrane samples.

\begin{tabular}{|c|c|c|c|c|c|c|c|c|c|c|c|c|c|c|c|c|}
\hline \multirow{3}{*}{$\begin{array}{l}\text { No } \\
\text { MS }\end{array}$} & \multirow{3}{*}{$\begin{array}{c}\begin{array}{c}\text { Membrane } \\
\text { Sample }\end{array} \\
\begin{array}{c}\text { Polymer/Sample } \\
\text { Name }\end{array}\end{array}$} & \multicolumn{2}{|c|}{ Membrane } & \multicolumn{6}{|c|}{ Membrane Properties } & \multicolumn{5}{|c|}{ VRFB Properties } & \multicolumn{2}{|c|}{ Reference } \\
\hline & & \multirow{2}{*}{ Struc } & \multirow{2}{*}{ Chem } & d & IEC $_{C}$ & IEC $_{A}$ & WU & $\mathbf{D}_{\mathrm{C}}$ & $\mathbf{D}_{\mathbf{r}}$ & CD & $\mathrm{CE}$ & VE & $\mathrm{EE}$ & $\mathrm{EE}_{\mathrm{r}}$ & \multirow{2}{*}{ Mem } & \multirow{2}{*}{ Pub } \\
\hline & & & & $\mu \mathrm{m}$ & $\mathrm{mmol} \mathrm{g}^{-1}$ & $\mathrm{mmol} \mathrm{g}^{-1}$ & wt. $\%$ & $\mathrm{~cm}^{2} \min ^{-1}$ & $\%$ & $\mathrm{~mA} \mathrm{~cm}^{-2}$ & $\%$ & $\%$ & $\%$ & & & \\
\hline 145 & SPEEK/DS92 & dho & CEM & 65 & 2.35 & - & 88 & $3.06 \times 10^{-6}$ & 0.243 & 200 & 93 & 82 & 77 & 1.048 & N212 & [55] \\
\hline 146 & SPEEK & dho & CEM & 100 & 1.8 & - & - & $2.43 \times 10^{-7}$ & 0.067 & 50 & 97 & 87 & 85 & 0.998 & N117 & {$[136]$} \\
\hline 147 & $\operatorname{SPEEK}(\mathrm{N} / \mathrm{S})$ & dhe & CEM & 100 & 1.67 & - & - & $1.93 \times 10^{-7}$ & 0.053 & 50 & 98 & 85 & 83 & 0.980 & N117 & [136] \\
\hline 148 & $\begin{array}{c}\text { SDPEEK } \\
(\mathrm{SD} 4-6-100)\end{array}$ & dho & CEM & 100 & 1.2 & - & 42.5 & $0.38 \times 10^{-7}$ & 0.027 & 50 & 98 & 90 & 88 & 1.046 & N115 & [101] \\
\hline 149 & $\begin{array}{c}\text { SDPEEK } \\
\text { (C-SD5-5-50) }\end{array}$ & dho & CEM & 50 & 1.65 & - & 29.8 & $0.48 \times 10^{-7}$ & 0.034 & 50 & 97 & 93 & 90 & 1.024 & N115 & [101] \\
\hline 150 & SPEEK & dho & CEM & 35 & 1.5 & - & 27 & $6.88 \times 10^{-7}$ & 0.433 & 40 & 98 & 91 & 89 & 1.145 & $\mathrm{~N} 112$ & [33] \\
\hline 151 & $\begin{array}{c}\text { SPEEK (PANI } \\
80 / 20)\end{array}$ & dhe & CEM & 37 & 1.44 & - & 21 & $2.67 \times 10^{-7}$ & 0.168 & 40 & 98 & 93 & 91 & 1.175 & N112 & {$[33]$} \\
\hline 152 & SPEEK & dho & CEM & 128 & - & - & 60.6 & $1.61 \times 10^{-6}$ & 0.797 & 40 & 93 & 95 & 88 & 1.060 & N115 & [142] \\
\hline 153 & $\begin{array}{c}\text { SPEEK } \\
\text { (SPEEK/RP) }\end{array}$ & dhe & CEM & 108 & - & - & 51.8 & $6.9 \times 10^{-7}$ & 0.342 & 120 & 98 & 81 & 80 & 1.060 & N115 & [142] \\
\hline
\end{tabular}


Table 7. Cont.

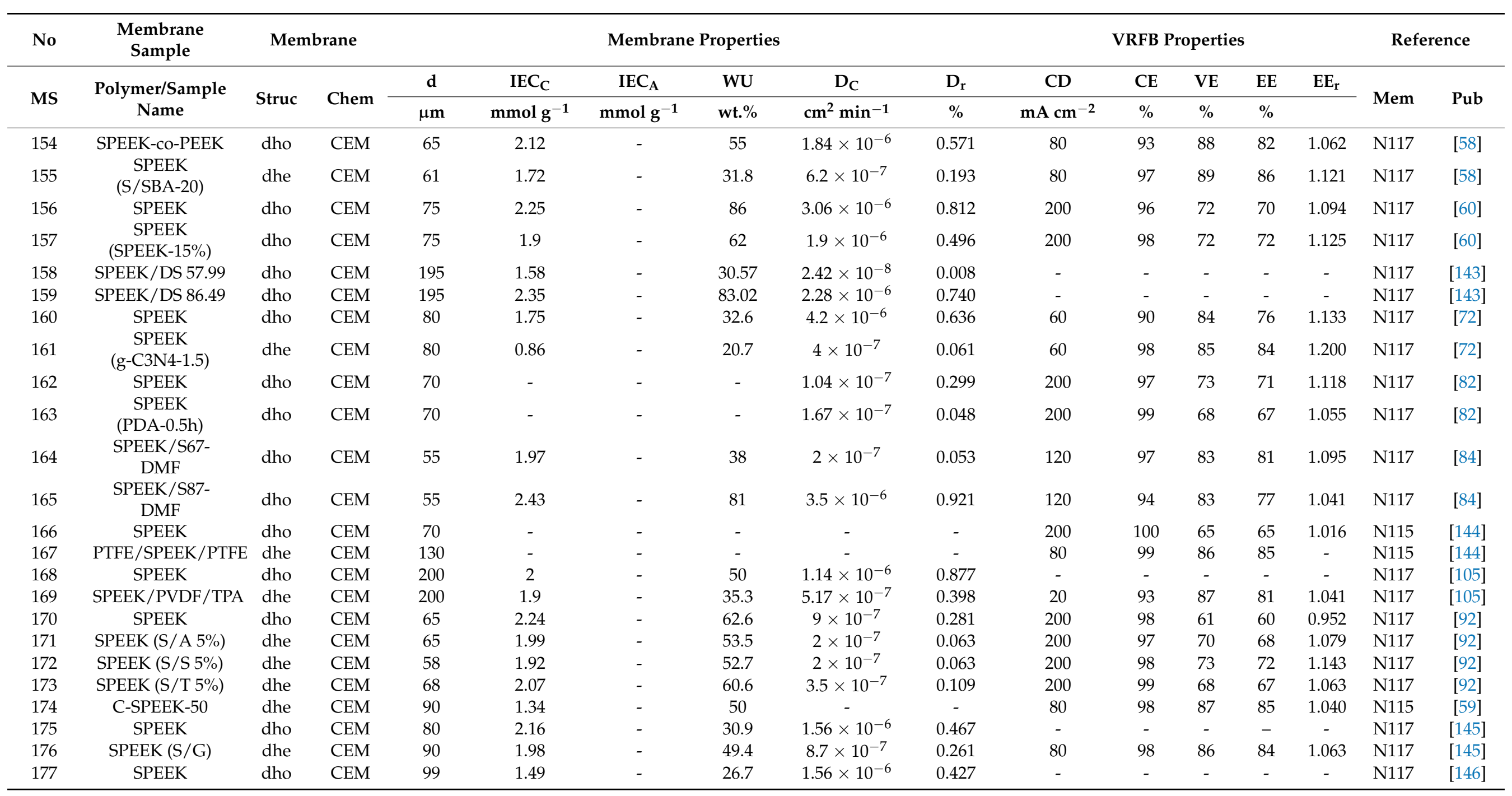


Table 7. Cont.

\begin{tabular}{|c|c|c|c|c|c|c|c|c|c|c|c|c|c|c|c|c|}
\hline \multirow{3}{*}{$\begin{array}{l}\text { No } \\
\text { MS }\end{array}$} & \multirow{3}{*}{$\begin{array}{c}\text { Membrane } \\
\text { Sample }\end{array}$} & \multicolumn{2}{|c|}{ Membrane } & \multicolumn{6}{|c|}{ Membrane Properties } & \multicolumn{5}{|c|}{ VRFB Properties } & \multicolumn{2}{|c|}{ Reference } \\
\hline & & \multirow{2}{*}{ Struc } & \multirow{2}{*}{ Chem } & d & IEC $_{C}$ & IEC $_{\mathrm{A}}$ & WU & $\mathbf{D}_{\mathrm{C}}$ & $\mathbf{D}_{\mathrm{r}}$ & CD & $\mathrm{CE}$ & VE & $\mathrm{EE}$ & $\mathrm{EE}_{\mathrm{r}}$ & \multirow{2}{*}{ Mem } & \multirow{2}{*}{ Pub } \\
\hline & & & & $\mu \mathrm{m}$ & $\mathrm{mmol} \mathrm{g}^{-1}$ & $\mathrm{mmol} \mathrm{g}^{-1}$ & wt. $\%$ & $\mathrm{~cm}^{2} \min ^{-1}$ & $\%$ & $\mathrm{~mA} \mathrm{~cm}^{-2}$ & $\%$ & $\%$ & $\%$ & & & \\
\hline 178 & SPEEK (S/OCN-1) & dhe & CEM & 100 & 1.56 & - & 48.2 & $9.09 \times 10^{-7}$ & 0.249 & 60 & 98 & 86 & 84 & 1.000 & N117 & {$[146]$} \\
\hline 179 & SPEEK & dho & CEM & 52 & 1.85 & - & 37.1 & $1.15 \times 10^{-6}$ & 0.330 & 200 & 98 & 68 & 67 & 1.047 & N117 & [38] \\
\hline 180 & SPEEK (S/GO 3) & dhe & CEM & 50 & 2.07 & - & 44.9 & $5.9 \times 10^{-7}$ & 0.171 & 200 & 98 & 72 & 71 & 1.109 & N117 & [38] \\
\hline 181 & SPEEK (S/P-0) & dho & CEM & - & 1.79 & - & 38.4 & $1.12 \times 10^{-7}$ & 1.172 & 50 & 90 & 91 & 82 & 1.007 & N117 & [66] \\
\hline 183 & SPEEK & dho & CEM & - & 2.03 & - & 43 & $4.2 \times 10^{-6}$ & 0.627 & 30 & 96 & 80 & 77 & 1.100 & N117 & [147] \\
\hline 184 & SPEEK (PPD-GO-1) & dhe & CEM & - & 1.08 & - & 22 & $1.2 \times 10^{-6}$ & 0.179 & 30 & 97 & 86 & 83 & 1.179 & N117 & [147] \\
\hline 185 & SPEEK & dho & CEM & 79 & 2.1 & - & 47.3 & $2.5 \times 10^{-7}$ & 0.104 & 80 & 97 & 76 & 74 & 1.021 & N117 & [40] \\
\hline 186 & SPEEK (S/P 15) & dhe & CEM & 74 & 1.79 & - & 39.6 & $1 \times 10^{-7}$ & 0.042 & 60 & 98 & 83 & 81 & 1.069 & N117 & {$[40]$} \\
\hline 187 & SPEEK & dho & CEM & 80 & - & - & - & $4.03 \times 10^{-7}$ & 0.490 & - & - & - & - & - & N212 & [148] \\
\hline 188 & SPEEK/SCCT & dhe & CEM & 90 & - & - & - & $3.22 \times 10^{-7}$ & 0.391 & 50 & 99 & 86 & 85 & 1.104 & N212 & [148] \\
\hline 189 & SPEEK (TiO2 5\%) & dhe & CEM & - & 1.5 & - & 23 & $2.45 \times 10^{-7}$ & 0.076 & 50 & 98 & 82 & 80.4 & 1.084 & N117 & [149] \\
\hline 190 & SPEEK (SPEEK-40) & dho & CEM & 90 & 1.45 & - & - & $0.36 \times 10^{-7}$ & 0.045 & 50 & 99 & 89 & 88 & 1.033 & N115 & [83] \\
\hline 191 & SPEEK (TPA/PP) & dhe & CEM & 240 & - & - & - & $4.78 \times 10^{-7}$ & 0.581 & 35.7 & 96 & 86 & 83 & 1.014 & N212 & [150] \\
\hline 193 & SPEEK (PSP) & dho & CEM & 75 & 0.74 & - & 7.8 & $1.37 \times 10^{-8}$ & 0.050 & 20 & 99 & 76 & 75 & 0.935 & N117 & [152] \\
\hline 194 & $\begin{array}{c}\text { PEEK-QADMPEK } \\
3\end{array}$ & dho & AEM & 43 & - & 1.75 & 18.8 & $7.64 \times 10^{-7}$ & 0.244 & 80 & 99 & 85 & 84 & 1.050 & N117 & [98] \\
\hline 195 & QPEK-C-TMA & dho & AEM & 40 & - & 1.4 & 36 & $8.2 \times 10^{-9}$ & 0.028 & 30 & 99 & 83 & 82 & - & N212 & [153] \\
\hline 196 & SPEKS/sGO 0.5 & dhe & CEM & - & 0.76 & - & 31 & $5 \times 10^{-8}$ & 0.161 & 40 & 99 & 83.3 & 82.5 & 1.12 & N212 & [154] \\
\hline 197 & SPEEK/ZC-GO-2 & dhe & AIEM & 75 & 1.87 & - & 36.5 & $12.7 \times 10^{-7}$ & 0.189 & 50 & 98.5 & 92.3 & 91.4 & 1.09 & N117 & [155] \\
\hline 198 & S/TPAM-1\% & dho & AIEM & 108 & 1.55 & - & 39.3 & $3.04 \times 10^{-7}$ & 0.074 & 60 & 97.5 & 86.6 & 83.8 & 1,018 & N115 & [156] \\
\hline 199 & SPEEK/L15 & dho & CEM & 81 & 1.11 & - & 29.62 & $1.7 \times 10^{-8}$ & 0.086 & 120 & 99.5 & 83.9 & 83.5 & - & N212 & [157] \\
\hline 200 & CrSPK45-S & dho & CEM & - & 1.67 & - & 22.16 & $6.1 \times 10^{-9}$ & 0.12 & 80 & 98 & 86.7 & 85 & 1.08 & N117 & [158] \\
\hline 201 & Q2-ADMPEK-4 & dho & AEM & - & - & 2.07 & 24,05 & - & - & 80 & 99 & 88.5 & 87.6 & 1.09 & N212 & [159] \\
\hline 202 & CQSPK-6 & dho & AEIM & & 0.95 & - & 21.6 & $1.05 \times 10^{-9}$ & 0.064 & 60 & 98.4 & 82.7 & 81.4 & 1.07 & N117 & [160] \\
\hline 203 & $\begin{array}{c}\text { SPAEK/Ce2Zr2O7 } \\
2 \%\end{array}$ & dhe & CEM & - & 1.31 & - & 52 & $1.29 \times 10^{-9}$ & 0.037 & 40 & 99.9 & 82.6 & 82.1 & 1.087 & N212 & [161] \\
\hline
\end{tabular}


Table 8. List of PSU, PPSU and PES-based hydro-carbon membrane samples.

\begin{tabular}{|c|c|c|c|c|c|c|c|c|c|c|c|c|c|c|c|c|}
\hline \multirow{3}{*}{$\begin{array}{l}\text { No } \\
\text { MS }\end{array}$} & \multirow{3}{*}{$\begin{array}{c}\begin{array}{c}\text { Membrane } \\
\text { Sample }\end{array} \\
\begin{array}{c}\text { Polymer/Sample } \\
\text { Name }\end{array} \\
\end{array}$} & \multicolumn{2}{|c|}{ Membrane } & \multicolumn{6}{|c|}{ Membrane Properties } & \multicolumn{5}{|c|}{ VRFB Properties } & \multicolumn{2}{|c|}{ Reference } \\
\hline & & \multirow{2}{*}{ Struc } & \multirow{2}{*}{ Chem } & d & IEC $_{C}$ & $\mathrm{IEC}_{\mathrm{A}}$ & WU & $\mathbf{D}_{\mathrm{C}}$ & $\mathrm{D}_{\mathbf{r}}$ & CD & $\mathrm{CE}$ & VE & $\mathrm{EE}$ & $\mathrm{EE}_{\mathbf{r}}$ & \multirow{2}{*}{ Mem } & \multirow{2}{*}{ Pub } \\
\hline & & & & $\mu \mathrm{m}$ & $\mathrm{mmol} \mathrm{g}^{-1}$ & $\mathrm{mmol} \mathrm{g}^{-1}$ & wt. $\%$ & $\mathrm{~cm}^{2} \min ^{-1}$ & $\%$ & $\mathrm{~mA} \mathrm{~cm}{ }^{-2}$ & $\%$ & $\%$ & $\%$ & & & \\
\hline 204 & $\begin{array}{c}\text { PSU / PVDF/imi } \\
\text { sIPN }\end{array}$ & dhe & AEM & - & - & - & 21 & - & - & 80 & 99 & 85 & 84 & - & - & [162] \\
\hline 205 & PSU /CMPSF 72 & sym & AEM & 45 & - & 1.51 & - & - & - & 80 & 99 & 87 & 86 & 1.048 & N115 & [102] \\
\hline 206 & PSU/ImPSf/SPEEK & dhe & AIEM & 65 & 2.04 & - & 56 & $1.5 \times 10^{-8}$ & 0.071 & 200 & 98 & 79 & 77 & 1.069 & $\mathrm{~N} 212$ & [55] \\
\hline 208 & PSU /PVP/PS M90 & asym & AIEM & 130 & - & - & - & - & - & 80 & 90 & 87 & 78 & - & - & [164] \\
\hline 209 & PSU/CPSF-Py & sym & AEM & 88 & - & - & - & - & - & 100 & 97 & 89 & 86 & 1.051 & N115 & [165] \\
\hline 210 & PSU/TMA & dho & AEM & 43 & - & - & - & $2.6 \times 10^{-8}$ & - & 30 & 96 & 88 & 85 & 1.012 & N212 & [166] \\
\hline 211 & $\begin{array}{c}\text { PSU / PSf-c-PTA- } \\
1.4\end{array}$ & dho & AEM & - & - & 1.7 & 36.7 & $2.57 \times 10^{-7}$ & 0.198 & 120 & 98.4 & 85.7 & 84.3 & 1.12 & N115 & [167] \\
\hline 212 & PSU/SPSF-62 & dho & CEM & 76 & 1.26 & - & 24.5 & $2.94 \times 10^{-6}$ & 0.438 & 100 & 98.8 & 87.2 & 86.2 & 1.14 & N117 & [168] \\
\hline 213 & $\begin{array}{c}\text { PSU/SPSF /g- } \\
\text { C3N4-1 }\end{array}$ & dhe & AIEM & 85 & 1.11 & - & 19.9 & $7 \times 10^{-7}$ & - & 100 & 98 & 89.1 & 87.3 & 1.15 & N117 & [169] \\
\hline 214 & PPSU/CMP-2 & dho & AEM & 42.5 & - & 1.95 & 35.3 & $1.5 \times 10^{-8}$ & 0.005 & 80 & 97 & 86 & 83 & 1.049 & N117 & [96] \\
\hline 216 & PPSU/AEM & dho & AEM & 50 & - & - & - & - & - & 60 & 100 & 70 & 70 & - & N212 & [170] \\
\hline 217 & PPSU/S-needle & dho & CEM & 115 & 1.95 & - & - & $2.07 \times 10^{-7}$ & 0.161 & 50 & 98 & 85 & 84 & 1.005 & N117 & [48] \\
\hline 218 & PPSU / BPSH35 & dho & CEM & - & 1.52 & - & 40 & $1.6 \times 10^{-9}$ & 0.123 & 80 & 99 & 76 & 75 & 1.042 & N212 & [49] \\
\hline 219 & PPSU/S2B2 & dho & AIEM* & 50 & 1.2 & - & 40.2 & & & 100 & 99 & 79 & 77 & 1.305 & N117 & [34] \\
\hline 220 & PES/PVP M3 & asym & AEM & 115 & - & - & - & $4 \times 10^{-6}$ & - & 80 & 93 & 85 & 79 & - & - & [171] \\
\hline 221 & $\begin{array}{c}\text { PES/SPEEK } \\
\text { M-35-13 }\end{array}$ & sym & CEM & 85 & - & - & - & - & - & 80 & 91 & 86 & 78 & - & - & [172] \\
\hline 222 & $\begin{array}{c}\text { PES/SPEEK } \\
\text { M-35-6 }\end{array}$ & asym & CEM & 160 & - & - & - & - & - & 80 & 92 & 85 & 78 & - & - & [173] \\
\hline 223 & PES/SPEEK M3 & dhe & CEM & 65 & - & - & - & - & - & 80 & 99 & 87 & 86 & 1.049 & N115 & [99] \\
\hline 224 & $\begin{array}{c}\text { PES/SPEEK/FT } \\
10 \%\end{array}$ & asym & CEM & 180 & - & - & - & $3.98 \times 10^{-6}$ & - & 80 & 95 & 86 & 82 & - & N115 & [174] \\
\hline 225 & $\begin{array}{c}\text { PES/SPEEK/N } \\
2.56\end{array}$ & asym & CEM & - & - & - & - & - & - & 80 & 99 & 87 & 86 & 1.012 & N115 & [81] \\
\hline 226 & $\begin{array}{c}\text { PES/SPEEK/PDDA } \\
7.5\end{array}$ & sym & AIEM & 132 & - & - & - & - & - & 80 & 98 & 92 & 90 & 1.071 & N115 & [175] \\
\hline 227 & PES/SPEEK/PPy & asym & AIEM* & 120 & - & - & - & - & - & 80 & 97 & 91 & 88 & 1.073 & N115 & [95] \\
\hline
\end{tabular}


Table 8. Cont.

\begin{tabular}{|c|c|c|c|c|c|c|c|c|c|c|c|c|c|c|c|c|}
\hline No & $\begin{array}{l}\text { Membrane } \\
\text { Sample }\end{array}$ & Men & rane & & & Membra & Properti & & & & RFB I & opertie & & & Ref & nce \\
\hline \multirow{2}{*}{ MS } & \multirow{2}{*}{$\begin{array}{c}\text { Polymer/Sample } \\
\text { Name }\end{array}$} & \multirow{2}{*}{ Struc } & \multirow{2}{*}{ Chem } & d & IEC $_{\mathrm{C}}$ & $\mathrm{IEC}_{\mathrm{A}}$ & WU & $\mathbf{D}_{\mathrm{C}}$ & $\mathbf{D}_{\mathrm{r}}$ & CD & $\mathrm{CE}$ & VE & EE & $\mathrm{EE}_{\mathbf{r}}$ & \multirow{2}{*}{ Mem } & \multirow{2}{*}{ Pub } \\
\hline & & & & $\mu \mathrm{m}$ & $\mathrm{mmol} \mathrm{g}^{-1}$ & $\mathrm{mmol} \mathrm{g}^{-1}$ & wt. $\%$ & $\mathrm{~cm}^{2} \min ^{-1}$ & $\%$ & $\mathrm{~mA} \mathrm{~cm}^{-2}$ & $\%$ & $\%$ & $\%$ & & & \\
\hline 228 & $\begin{array}{c}\mathrm{PES} / \mathrm{SPEEK} / \mathrm{SiO} 2 \\
\text { M2 }\end{array}$ & dhe & CEM & 125 & - & - & 25 & - & - & 80 & 82 & 87 & 94 & 0.989 & N115 & [93] \\
\hline 229 & $\begin{array}{c}\text { PES/SPEEK/ZSM- } \\
35\end{array}$ & asym & CEM & - & - & - & - & - & - & 80 & 98 & 93 & 91 & 1.109 & N115 & [97] \\
\hline 231 & SPES & dho & CEM & - & 2.07 & - & 121.93 & $2.5 \times 10^{-6}$ & 0.809 & - & - & - & - & - & - & [143] \\
\hline 232 & SPAES S/N & dhe & CEM & - & - & - & - & - & - & 200 & 99 & 75 & 74 & 1.035 & N115 & [69] \\
\hline 233 & SPES (IL-30) & asym & CEM & - & - & - & - & $1.41 \times 10^{-8}$ & - & 140 & 99 & 80.13 & 79.3 & 0.98 & $\mathrm{~N} 212$ & {$[177]$} \\
\hline 234 & MD2.0-10 & dhe & AEIM & - & - & - & - & $1.7 \times 10^{-7}$ & 0.134 & 80 & 99.3 & 82.6 & 82 & 1.025 & $\mathrm{~N} 115$ & [178] \\
\hline
\end{tabular}

Table 9. List of fluorenyl-ether-based hydro-carbon membrane samples.

\begin{tabular}{|c|c|c|c|c|c|c|c|c|c|c|c|c|c|c|c|c|}
\hline \multirow{3}{*}{$\begin{array}{l}\text { No } \\
\text { MS }\end{array}$} & \multirow{3}{*}{$\begin{array}{c}\begin{array}{c}\text { Membrane } \\
\text { Sample }\end{array} \\
\begin{array}{c}\text { Polymer/Sample } \\
\text { Name }\end{array}\end{array}$} & \multicolumn{2}{|c|}{ Membrane } & \multicolumn{6}{|c|}{ Membrane Properties } & \multicolumn{5}{|c|}{ VRFB Properties } & \multicolumn{2}{|c|}{ Reference } \\
\hline & & \multirow{2}{*}{ Struc } & \multirow{2}{*}{ Chem } & d & IEC $_{C}$ & IEC $_{A}$ & WU & $\mathbf{D}_{\mathrm{C}}$ & $\mathbf{D}_{\mathrm{r}}$ & CD & $\mathrm{CE}$ & VE & $\mathrm{EE}$ & $\mathrm{EE}_{\mathrm{r}}$ & \multirow{2}{*}{ Mem } & \multirow{2}{*}{ Pub } \\
\hline & & & & $\mu \mathrm{m}$ & $\mathrm{mmol} \mathrm{g}^{-1}$ & $\mathrm{mmol} \mathrm{g}^{-1}$ & wt. $\%$ & $\mathrm{~cm}^{2} \min ^{-1}$ & $\%$ & $\mathrm{~mA} \mathrm{~cm}^{-2}$ & $\%$ & $\%$ & $\%$ & & & \\
\hline 235 & QA-PFE & dho & AEM & 56 & - & 2.0 & - & - & - & 60 & 100 & 70 & 70 & 1.0 & $\mathrm{~N} 212$ & [37] \\
\hline 236 & SPECIAL & dho & CEM & 112.5 & 1.92 & - & 29 & - & 0.25 & 50 & 66.2 & 73.7 & 48.8 & - & N117 & {$[179]$} \\
\hline 237 & F-SPFEK & dho & CEM & 112.5 & 1.88 & - & 33 & - & 0.75 & 50 & 76.1 & 80.3 & 61.1 & - & N117 & [179] \\
\hline 238 & F-SPFEK-APTES & dho & CEM & 112.5 & 1.75 & - & 26 & - & 0.50 & 50 & 80.4 & 79.7 & 64.1 & - & N117 & [179] \\
\hline 240 & SPFEK/3\%SIO2 & dhe & CEM & 155 & 1.83 & - & 36.6 & - & 0.29 & 60 & 87.5 & - & - & - & N117 & {$[180]$} \\
\hline 241 & SPECIAL & dho & CEM & 180 & 1.92 & - & 27.8 & $9.85 \times 10^{-7}$ & 0.40 & 40 & 80.3 & 64.6 & 51.9 & - & N117 & {$[180]$} \\
\hline 242 & SPECIAL & dho & CEM & - & 1.87 & - & - & - & - & - & - & - & - & - & N117 & [181] \\
\hline 243 & SPFEK/5ZrPSPP & dhe & CEM & - & 1.96 & - & - & - & - & 50 & 89 & 60.7 & 54 & - & N117 & [181] \\
\hline 244 & $\begin{array}{c}\text { SPFEK- } \\
\text { [PDDA/PSS]n2 }\end{array}$ & dhe & AIEM & 130 & - & - & - & - & - & - & - & - & - & - & N117 & {$[182]$} \\
\hline 245 & $\begin{array}{l}\text { SPFEK } 20.7 \\
{[\text { PDA/PSS]2 }}\end{array}$ & sym & AIEM & 151 & - & - & - & $5.92 \times 10^{-7}$ & 0.36 & - & - & - & - & - & N115 & {$[183]$} \\
\hline 246 & SPECIAL & dho & CEM & 160 & 1.57 & - & 36.5 & $2.67 \times 10^{-7}$ & 0.13 & - & - & - & - & - & N115 & {$[184]$} \\
\hline
\end{tabular}


Table 9. Cont.

\begin{tabular}{|c|c|c|c|c|c|c|c|c|c|c|c|c|c|c|c|c|}
\hline No & $\begin{array}{l}\text { Membrane } \\
\text { Sample }\end{array}$ & Mem & rane & & & Membran & roperti & & & & RFB & perti & & & Ref & nce \\
\hline \multirow{2}{*}{ MS } & \multirow{2}{*}{$\begin{array}{c}\text { Polymer/Sample } \\
\text { Name }\end{array}$} & \multirow{2}{*}{ Struc } & \multirow{2}{*}{ Chem } & d & IEC $_{\mathrm{C}}$ & $\mathrm{IEC}_{\mathrm{A}}$ & WU & $\mathrm{D}_{\mathrm{C}}$ & $D_{r}$ & $\mathrm{CD}$ & $\mathrm{CE}$ & $\mathrm{VE}$ & $\mathrm{EE}$ & $\mathrm{EE}_{\mathrm{r}}$ & \multirow{2}{*}{ Mem } & \multirow{2}{*}{ Pub } \\
\hline & & & & $\mu \mathrm{m}$ & $\mathrm{mmol} \mathrm{g}^{-1}$ & $\mathrm{mmol} \mathrm{g}^{-1}$ & wt. $\%$ & $\mathrm{~cm}^{2} \min ^{-1}$ & $\%$ & $\mathrm{~mA} \mathrm{~cm}{ }^{-2}$ & $\%$ & $\%$ & $\%$ & & & \\
\hline 247 & SPFEKA $10 \%$. & dho & AIEM & 160 & 1.52 & - & 30.6 & $1.56 \times 10^{-7}$ & 0.08 & - & - & - & - & - & N115 & [184] \\
\hline 248 & SPFEKA-20\% & dho & AIEM & 160 & 1.47 & - & 30.9 & $0.88 \times 10^{-7}$ & 0.04 & - & - & - & - & - & N115 & [184] \\
\hline 249 & HSPAEK & dho & CEM & 60 & 1.72 & - & 38.5 & $5.5 \times 10^{-7}$ & 0.16 & 80 & 98 & 85 & 83 & 1.05 & N117 & [90] \\
\hline
\end{tabular}

Table 10. List of poly(phenylene ether)-based hydro-carbon membrane samples.

\begin{tabular}{|c|c|c|c|c|c|c|c|c|c|c|c|c|c|c|c|c|}
\hline No & $\begin{array}{c}\text { Membrane } \\
\text { Sample }\end{array}$ & Men & rane & & & Membran & roperti & & & & RFB & pert & & & $\operatorname{Ref}$ & ence \\
\hline \multirow{2}{*}{ MS } & \multirow{2}{*}{$\begin{array}{c}\text { Polymer/Sample } \\
\text { Name }\end{array}$} & \multirow{2}{*}{ Struc } & \multirow{2}{*}{ Chem } & d & IEC $_{\mathrm{C}}$ & $\mathrm{IEC}_{\mathrm{A}}$ & WU & $\mathbf{D}_{\mathrm{C}}$ & $\mathrm{D}_{\mathrm{r}}$ & $\mathrm{CD}$ & $\mathrm{CE}$ & VE & EE & $\mathrm{EE}_{\mathrm{r}}$ & \multirow{2}{*}{ Mem } & \multirow{2}{*}{ Pub } \\
\hline & & & & $\mu \mathrm{m}$ & $\mathrm{mmol} \mathrm{g}^{-1}$ & $\mathrm{mmol} \mathrm{g}^{-1}$ & wt. $\%$ & $\mathrm{~cm}^{2} \min ^{-1}$ & $\%$ & $\mathrm{~mA} \mathrm{~cm}^{-2}$ & $\%$ & $\%$ & $\%$ & & & \\
\hline 250 & BrPPO/Py-42 & dho & AEM & 50 & - & - & 13 & $0.12 \times 10^{-7}$ & 0.02 & 100 & 98.1 & 84 & 82 & 0.932 & $\mathrm{~N} 212$ & [35] \\
\hline 251 & $\mathrm{BrPPO} / \mathrm{Py}-56$ & dho & $\mathrm{AEM}$ & 50 & - & - & 18 & $0.2 \times 10^{-7}$ & 0.03 & 100 & 97.7 & 94 & 92 & 1.045 & $\mathrm{~N} 212$ & [35] \\
\hline 252 & $\mathrm{BrPPO} / \mathrm{Py}-70$ & dho & $\mathrm{AEM}$ & 50 & - & - & 21 & $0.36 \times 10^{-7}$ & 0.05 & 100 & 96.7 & 90 & 87 & 0.989 & $\mathrm{~N} 212$ & [35] \\
\hline 253 & SPPO-GO & dhe & CEM & - & 1.17 & - & 16.3 & $1.1 \times 10^{-8}$ & 0.05 & 40 & 98 & 71 & 69.6 & - & N212 & [185] \\
\hline
\end{tabular}

Table 11. List of other hydro-carbon-based membrane samples.

\begin{tabular}{|c|c|c|c|c|c|c|c|c|c|c|c|c|c|c|c|c|}
\hline No & $\begin{array}{l}\text { Membrane } \\
\text { Sample }\end{array}$ & Men & orane & \multirow{3}{*}{$\begin{array}{c}\mathrm{d} \\
\mu \mathrm{m}\end{array}$} & \multicolumn{4}{|c|}{ Membrane Properties } & \multirow{3}{*}{$\begin{array}{c}D_{r} \\
\%\end{array}$} & \multicolumn{5}{|c|}{ VRFB Properties } & \multicolumn{2}{|c|}{ Reference } \\
\hline \multirow{2}{*}{ MS } & \multirow{2}{*}{$\begin{array}{c}\text { Polymer/Sample } \\
\text { Name }\end{array}$} & \multirow{2}{*}{ Struc } & \multirow{2}{*}{ Chem } & & IEC $_{C}$ & $\mathrm{IEC}_{\mathrm{A}}$ & WU & $\mathbf{D}_{\mathrm{C}}$ & & $\mathrm{CD}$ & $\mathrm{CE}$ & $\mathrm{VE}$ & $\mathrm{EE}$ & $\mathrm{EE}_{\mathbf{r}}$ & \multirow{2}{*}{ Mem } & \multirow{2}{*}{ Pub } \\
\hline & & & & & $\mathrm{mmol} \mathrm{g}^{-1}$ & $\mathrm{mmol} \mathrm{g}^{-1}$ & wt. $\%$ & $\mathrm{~cm}^{2} \min ^{-1}$ & & $\mathrm{~mA} \mathrm{~cm}{ }^{-2}$ & $\%$ & $\%$ & $\%$ & & & \\
\hline 254 & QPTM & dho & AEM & 89 & - & 2.08 & 8.8 & $1.19 \times 10^{-7}$ & 0.034 & 50 & 100 & 75 & 75 & 1.033 & N117 & [41] \\
\hline 255 & QVTD 2-3 & dho & AEM & - & - & - & - & - & - & 40 & 95 & 80 & 75 & - & - & [186] \\
\hline 256 & VBC AVSH-3 & dho & AEM & - & - & - & - & - & - & 40 & 95 & 75 & 75 & - & - & [187] \\
\hline 257 & PVC/silica & sym & - & 390 & - & - & - & - & - & 40 & 89 & 88 & 78 & 0.907 & N115 & [188] \\
\hline 259 & DHIM-375 & dho & CEM & 100 & 0.69 & - & 31 & $1.56 \times 10^{-7}$ & 0.052 & 20 & 91 & 80 & 72 & - & N117 & [190] \\
\hline
\end{tabular}


Table 11. Cont.

\begin{tabular}{|c|c|c|c|c|c|c|c|c|c|c|c|c|c|c|c|c|}
\hline No & $\begin{array}{l}\text { Membrane } \\
\text { Sample }\end{array}$ & Men & rane & & & Membran & roperti & & & & RFB I & operti & & & Ref & ence \\
\hline \multirow{2}{*}{ MS } & \multirow{2}{*}{$\begin{array}{c}\text { Polymer/Sample } \\
\text { Name }\end{array}$} & \multirow{2}{*}{ Struc } & \multirow{2}{*}{ Chem } & $\mathrm{d}$ & IEC $_{C}$ & $\mathrm{IEC}_{\mathrm{A}}$ & WU & $\mathrm{D}_{\mathrm{C}}$ & $D_{r}$ & $\mathrm{CD}$ & $\mathrm{CE}$ & VE & $\mathrm{EE}$ & $\mathrm{EE}_{\mathrm{r}}$ & \multirow{2}{*}{ Mem } & \multirow{2}{*}{ Pub } \\
\hline & & & & $\mu \mathrm{m}$ & $\mathrm{mmol} \mathrm{g}^{-1}$ & $\mathrm{mmol} \mathrm{g}^{-1}$ & wt. $\%$ & $\mathrm{~cm}^{2} \min ^{-1}$ & $\%$ & $\mathrm{~mA} \mathrm{~cm}^{-2}$ & $\%$ & $\%$ & $\%$ & & & \\
\hline 260 & ZРРТ-6 & dho & AIEM & 80 & - & 1.22 & 30 & - & - & 50 & 98 & 80 & 78 & 1.05 & N117 & [57] \\
\hline 261 & PIM-1 & asym & - & “0.75” & - & - & - & - & - & 20 & 97.1 & 92.5 & 89.9 & 1.2 & N112- & [191] \\
\hline
\end{tabular}

Table 12. List of PBI-based N-heterocycle membrane samples.

\begin{tabular}{|c|c|c|c|c|c|c|c|c|c|c|c|c|c|c|c|c|}
\hline \multirow{3}{*}{$\begin{array}{l}\text { No } \\
\text { MS }\end{array}$} & \multirow{3}{*}{$\begin{array}{c}\begin{array}{c}\text { Membrane } \\
\text { Sample }\end{array} \\
\begin{array}{c}\text { Polymer/Sample } \\
\text { Name }\end{array} \\
\end{array}$} & \multicolumn{2}{|c|}{ Membrane } & \multicolumn{6}{|c|}{ Membrane Properties } & \multicolumn{5}{|c|}{ VRFB Properties } & \multicolumn{2}{|c|}{ Reference } \\
\hline & & \multirow{2}{*}{ Struc } & \multirow{2}{*}{ Chem } & d & IEC $_{C}$ & $\mathrm{IEC}_{\mathrm{A}}$ & WU & $\mathbf{D}_{\mathrm{C}}$ & $\mathrm{D}_{\mathrm{r}}$ & CD & $\mathrm{CE}$ & VE & EE & $\mathrm{EE}_{\mathrm{r}}$ & \multirow{2}{*}{ Mem } & \multirow{2}{*}{ Pub } \\
\hline & & & & $\mu \mathrm{m}$ & $\mathrm{mmol} \mathrm{g}^{-1}$ & $\mathrm{mmol} \mathrm{g}^{-1}$ & wt. $\%$ & $\mathrm{~cm}^{2} \min ^{-1}$ & $\%$ & $\mathrm{~mA} \mathrm{~cm}^{-2}$ & $\%$ & $\%$ & $\%$ & & & \\
\hline 262 & $\mathrm{mPBI}$ & dho & AEM & 27 & - & - & - & $2.86 \times 10^{-9}$ & 0.008 & 50 & 99.5 & 80.4 & 80 & 0.941 & N115 & [65] \\
\hline 263 & BlpPBI & dho & AEM & 27 & - & - & - & $3.45 \times 10^{-8}$ & 0.099 & 50 & 99 & 88.4 & 87.5 & 1.029 & N115 & [65] \\
\hline 264 & mPBI-15 & dho & AEM & 15 & - & - & - & 0 & - & 120 & 99.8 & 68 & 67.9 & 0.893 & $\mathrm{~N} 212$ & [192] \\
\hline 265 & mPBI-35 & dho & AEM & 35 & - & - & - & 0 & - & 120 & 99 & 53 & 52.5 & 0.691 & $\mathrm{~N} 212$ & [192] \\
\hline 267 & PBI-0\% & dho & AEM & 16 & - & - & - & - & - & 40 & 95 & 50 & 47 & 0.595 & N117 & [70] \\
\hline 268 & PBI $10 \%$. & sym & AEM & 45 & - & - & - & $1.17 \times 10^{-7}$ & - & 40 & 99 & 79 & 78 & 0.987 & N117 & [70] \\
\hline 269 & PBI-200 & asym & AEM & 100 & - & - & - & $3 \times 10^{-9}$ & - & 80 & 99 & 83 & 82 & 1.206 & $\mathrm{~N} 211$ & [194] \\
\hline 270 & PBI-O/PBI-34 & sym & $\mathrm{AEM}$ & 34 & - & - & - & - & - & 80 & 99 & 91 & 90 & 1.092 & $\mathrm{~N} 115$ & [195] \\
\hline 271 & CSOPBI-NH2 $(9 / 1)$ & dho & AIEM $^{*}$ & 55 & 0.24 & - & 47.4 & $6 \times 10^{-9}$ & 0.001 & 60 & 98 & 86 & 84 & 1.024 & N117 & [87] \\
\hline 272 & $-6 \mathrm{~F}-\mathrm{co}-10 \% \mathrm{BI}$ & dho & AIEM $^{*}$ & 64 & 1.56 & - & - & $2.24 \times 10^{-11}$ & - & 30 & 99 & 91 & 90 & 1.027 & N117 & [63] \\
\hline 273 & $-6 \mathrm{~F}-\mathrm{co}-10 \% \mathrm{BI}-\mathrm{cld}$ & dho & AIEM $^{*}$ & 65 & 1.50 & - & - & $1.28 \times 10^{-11}$ & - & 30 & 99 & 90 & 89 & 1.018 & N117 & [63] \\
\hline 274 & FPAE-6F-PBI S1B1 & dhe & AIEM $^{*}$ & 50 & 1.02 & - & 23.8 & - & - & 100 & 100 & 64 & 64 & 1.085 & N117 & [34] \\
\hline 275 & B20N10 & dho & AEIM & 30 & - & - & 23.6 & $1.95 \times 10^{-9}$ & 0.006 & 80 & 100 & 82.2 & 82.2 & 1.068 & N115 & [196] \\
\hline 277 & $0,7 \mu \mathrm{m}$ PBI & asym & AEM & 30 & - & - & - & - & - & 120 & 98.5 & 85 & 83 & 1.034 & $\mathrm{~N} 212$ & [198] \\
\hline 278 & PWN/F6PBI(9/1) & dho & AIEM & 45 & 1.51 & - & - & - & - & 40 & 99 & 81 & 81 & 1 & $\mathrm{~N} 212$ & [199] \\
\hline 279 & PVDF-PBI & asym & AEM & - & - & - & - & - & - & 60 & 98.4 & 83.3 & 82 & 1.03 & N117 & [200] \\
\hline 280 & sPBI & dho & AEIM & 220.2 & - & - & 58.1 & $5.74 \times 10^{-7}$ & - & 242 & 93 & 86 & 81 & - & $\mathrm{N} 212$ & [201] \\
\hline 281 & $\mathrm{PE} / \mathrm{PBI}$ & dhe & AEM & 25 & - & - & 20.9 & $5.04 \times 10^{-7}$ & 0.346 & 200 & 99 & 81 & 80.11 & 1.03 & $\mathrm{~N} 212$ & [202] \\
\hline
\end{tabular}


Table 13. List of poly(phthalazinone ether)-based $N$-heterocycle membrane samples.

\begin{tabular}{|c|c|c|c|c|c|c|c|c|c|c|c|c|c|c|c|c|}
\hline \multirow{3}{*}{$\begin{array}{l}\text { No } \\
\text { MS }\end{array}$} & \multirow{3}{*}{$\begin{array}{c}\begin{array}{c}\text { Membrane } \\
\text { Sample }\end{array} \\
\begin{array}{c}\text { Polymer/Sample } \\
\text { Name }\end{array}\end{array}$} & \multicolumn{2}{|c|}{ Membrane } & \multicolumn{6}{|c|}{ Membrane Properties } & \multicolumn{5}{|c|}{ VRFB Properties } & \multicolumn{2}{|c|}{ Reference } \\
\hline & & \multirow{2}{*}{ Struc } & \multirow{2}{*}{ Chem } & $\mathrm{d}$ & IEC $_{\mathrm{C}}$ & IEC $_{\mathrm{A}}$ & WU & $\mathrm{D}_{\mathrm{C}}$ & $\mathrm{D}_{\mathrm{r}}$ & $\mathrm{CD}$ & $\mathrm{CE}$ & VE & EE & $\mathrm{EE}_{\mathrm{r}}$ & \multirow{2}{*}{ Mem } & \multirow{2}{*}{ Pub } \\
\hline & & & & $\mu \mathrm{m}$ & $\mathrm{mmol} \mathrm{g}^{-1}$ & $\mathrm{mmol} \mathrm{g}^{-1}$ & wt. $\%$ & $\mathrm{~cm}^{2} \min ^{-1}$ & $\%$ & $\mathrm{~mA} \mathrm{~cm}^{-2}$ & $\%$ & $\%$ & $\%$ & & & \\
\hline 282 & PyPPEK-2 & dho & AEM & - & - & 1.4 & 17.4 & - & - & 60 & 99 & 85 & 84 & 1.000 & N117 & [203] \\
\hline 283 & QAPPEK-2 & dho & $\mathrm{AEM}$ & - & - & 1.5 & 21 & - & - & 60 & 99 & 83 & 82 & 0.964 & N117 & [203] \\
\hline 284 & QAPPEKK & dho & AEM & - & - & 1.56 & - & - & - & 40 & 99 & 89 & 88 & 1.026 & N117 & [103] \\
\hline 285 & PyPРEKK-4 & dho & AEM & 42 & - & 1.55 & 16.5 & - & - & 40 & 98 & 90 & 89 & 1.034 & N117 & [103] \\
\hline 287 & QAPPEKK-4 & dho & AEM & 50 & - & 1.56 & 20.8 & - & - & 20 & 98 & 93 & 91.3 & 1.016 & N117 & [100] \\
\hline 288 & SPEC & dho & CEM & 200 & 1.272 & - & 32.34 & $2.77 \times 10^{-7}$ & 0.024 & 60 & 99 & 69 & 68 & 0.919 & N117 & [80] \\
\hline 289 & SPPEK TPA-17 & dhe & CEM & 200 & 1.142 & - & 33.28 & $5.75 \times 10^{-7}$ & 0.049 & 60 & 99 & 76 & 75 & 1.010 & N117 & [80] \\
\hline 290 & SPPEK/WO3 & dhe & CEM & 210 & - & - & 48.15 & $3.97 \times 10^{-7}$ & 0.034 & 50 & 98 & 80 & 79 & 1.032 & N117 & [79] \\
\hline 291 & SPPEK P-90 & dho & CEM & 53 & 1.51 & - & 23.2 & - & - & 40 & 98 & 89 & 87 & 1.023 & N115 & [61] \\
\hline 292 & SPPES/SP-02 & dho & CEM & 260 & 1.42 & - & 17.42 & $1.24 \times 10^{-7}$ & 0.055 & 40 & 93 & 73 & 68 & 1.004 & N117 & [205] \\
\hline
\end{tabular}

Table 14. List of poly(phthalazinone ether)-based N-heterocycle membrane samples.

\begin{tabular}{|c|c|c|c|c|c|c|c|c|c|c|c|c|c|c|c|c|}
\hline \multirow{3}{*}{$\begin{array}{l}\text { No } \\
\text { MS }\end{array}$} & \multirow{3}{*}{$\begin{array}{c}\begin{array}{c}\text { Membrane } \\
\text { Sample }\end{array} \\
\begin{array}{c}\text { Polymer/Sample } \\
\text { Name }\end{array} \\
\end{array}$} & \multicolumn{2}{|c|}{ Membrane } & \multicolumn{6}{|c|}{ Membrane Properties } & \multicolumn{5}{|c|}{ VRFB Properties } & \multicolumn{2}{|c|}{ Reference } \\
\hline & & \multirow{2}{*}{ Struc } & \multirow{2}{*}{ Chem } & d & IEC $_{C}$ & IEC $_{A}$ & WU & $\mathbf{D}_{\mathrm{C}}$ & $\mathrm{D}_{\mathrm{r}}$ & CD & CE & VE & $\mathrm{EE}$ & $\mathrm{EE}_{\mathrm{r}}$ & \multirow{2}{*}{ Mem } & \multirow{2}{*}{ Pub } \\
\hline & & & & $\mu \mathrm{m}$ & $\mathrm{mmol} \mathrm{g}^{-1}$ & $\mathrm{mmol} \mathrm{g}^{-1}$ & wt. $\%$ & $\mathrm{~cm}^{2} \min ^{-1}$ & $\%$ & $\mathrm{~mA} \mathrm{~cm}^{-2}$ & $\%$ & $\%$ & $\%$ & & & \\
\hline 293 & SPI (ODA) & dho & CEM & 60 & 1.2 & - & 21.93 & $2.17 \times 10^{-7}$ & 0.127 & - & - & - & - & - & N117 & [74] \\
\hline 294 & bSPI (APABI) & dho & AIEM* & 54 & 1.3 & - & 28.80 & $1.75 \times 10^{-7}$ & 0.102 & - & - & - & - & - & N117 & [74] \\
\hline 295 & bSPI(MDA) & dho & CEM & 55 & 1.37 & - & 34.88 & $4.43 \times 10^{-7}$ & 0.259 & - & - & - & - & - & N117 & {$[74]$} \\
\hline 296 & bSPI(BAPP) & dho & CEM & 57 & 1.14 & - & 20.03 & $2.89 \times 10^{-7}$ & 0.169 & 120 & 99 & 64 & 63 & 1.018 & N117 & {$[74]$} \\
\hline 298 & s-FSPI & dho & CEM & - & 1.50 & - & 17.78 & $7.4 \times 10^{-8}$ & 0.055 & 60 & 100 & 77 & 77 & 1.160 & N115 & [207] \\
\hline 299 & 6F-SPI-50 & dho & CEM & - & - & - & - & $2.27 \times 10^{-7}$ & 0.172 & 60 & 99.5 & 72.4 & 72 & 1.091 & N115 & [75] \\
\hline 300 & SPI & dho & CEM & 50 & 1.58 & - & 25.7 & $2.25 \times 10^{-7}$ & 0.165 & 60 & 98 & 78 & 76 & 1.086 & N115 & {$[78]$} \\
\hline 301 & 6F-s-bSPI & dho & CEM & 35 & 1.54 & - & 16.5 & $1.18 \times 10^{-7}$ & 0.087 & 60 & 100 & 79 & 79 & 1.129 & N115 & [78] \\
\hline 302 & SPI & dho & CEM & 69 & 1.75 & - & 39.92 & $1.89 \times 10^{-7}$ & 0.111 & 70 & 93 & 70 & 65 & 0.956 & N117 & [104] \\
\hline 303 & SPI/AlOOH-10 & dhe & CEM & 58 & 0.95 & - & 48.59 & $1.14 \times 10^{-7}$ & 0.067 & 70 & 96 & 73 & 70 & 1.029 & N117 & [104] \\
\hline
\end{tabular}


Table 14. Cont.

\begin{tabular}{|c|c|c|c|c|c|c|c|c|c|c|c|c|c|c|c|c|}
\hline \multirow{3}{*}{$\begin{array}{l}\text { No } \\
\text { MS }\end{array}$} & \multirow{3}{*}{$\begin{array}{c}\begin{array}{c}\text { Membrane } \\
\text { Sample }\end{array} \\
\begin{array}{c}\text { Polymer/Sample } \\
\text { Name }\end{array}\end{array}$} & \multicolumn{2}{|c|}{ Membrane } & \multicolumn{6}{|c|}{ Membrane Properties } & \multicolumn{5}{|c|}{ VRFB Properties } & \multicolumn{2}{|c|}{ Reference } \\
\hline & & \multirow{2}{*}{ Struc } & \multirow{2}{*}{ Chem } & $\mathrm{d}$ & IEC $_{C}$ & $\mathrm{IEC}_{\mathrm{A}}$ & WU & $\mathbf{D}_{\mathrm{C}}$ & $\mathrm{D}_{\mathbf{r}}$ & CD & $\mathrm{CE}$ & VE & $\mathrm{EE}$ & $\mathrm{EE}_{\mathbf{r}}$ & \multirow{2}{*}{ Mem } & \multirow{2}{*}{ Pub } \\
\hline & & & & $\mu \mathrm{m}$ & $\mathrm{mmol} \mathrm{g}^{-1}$ & $\mathrm{mmol} \mathrm{g}^{-1}$ & wt. $\%$ & $\mathrm{~cm}^{2} \min ^{-1}$ & $\%$ & $\mathrm{~mA} \mathrm{~cm}{ }^{-2}$ & $\%$ & $\%$ & $\%$ & & & \\
\hline 304 & SPI(APABI) & dho & AIEM* & 65 & 1.24 & - & 22.79 & $7.2 \times 10^{-8}$ & 0.042 & 30 & 100 & 77 & 77 & 1.069 & N117 & [208] \\
\hline 305 & SPI(BAPP) & dho & CEM & 62 & 1.49 & - & 27.08 & $4.8 \times 10^{-8}$ & 0.028 & 30 & 100 & 79 & 79 & 1.097 & N117 & [208] \\
\hline 306 & SPI(MDA) & dho & CEM & 64 & 1.48 & - & 26.94 & $2.36 \times 10^{-7}$ & 0.138 & 30 & 98 & 72 & 71 & 0.986 & N117 & [208] \\
\hline 307 & SPI & dho & CEM & 45 & 1.61 & - & 41.40 & $1.89 \times 10^{-7}$ & 0.123 & 40 & 94 & 92 & 87 & 0.998 & N117 & [94] \\
\hline 309 & SPI & dho & CEM & 65 & 1.58 & - & 37.14 & $2.37 \times 10^{-7}$ & 0.139 & - & - & - & - & - & N117 & [209] \\
\hline 310 & SPI/MoS2 & dhe & CEM & 65 & 1.25 & - & 29.36 & $2.02 \times 10^{-7}$ & 0.119 & 80 & 95 & 65 & 62 & 1.016 & N117 & [209] \\
\hline 311 & SPI/s-MoS2 & dhe & CEM & 66 & 1.70 & - & 32.20 & $1.23 \times 10^{-7}$ & 0.072 & 80 & 96 & 66 & 63 & 1.033 & N117 & [209] \\
\hline 312 & SPI & dho & CEM & 50 & 1.25 & - & 54.7 & $2.6 \times 10^{-6}$ & 0.388 & 40 & 89 & 77 & 69 & 1.045 & N117 & [210] \\
\hline 313 & SPI/PEI-GO-2 & dhe & AIEM* & 50 & 1.16 & - & 44.2 & $7 \times 10^{-7}$ & 0.104 & 40 & 95 & 82 & 77 & 1.167 & N117 & [210] \\
\hline 314 & SPI & dho & CEM & 55 & 1.40 & - & 38.46 & $1.9 \times 10^{-7}$ & 0.111 & - & - & - & - & - & N117 & [54] \\
\hline 315 & $\mathrm{SPI} / \mathrm{TiO} 2$ & dhe & CEM & 49 & 1.24 & - & 32.94 & $2.02 \times 10^{-7}$ & 0.118 & 70 & 97 & 72 & 69 & 1.022 & N117 & [54] \\
\hline 316 & SPI & dho & CEM & 50 & 1.25 & - & 54.7 & $2.6 \times 10^{-6}$ & 0.388 & 80 & 94 & 65 & 63 & 1.050 & N117 & [211] \\
\hline 319 & SPI/ZrO2-15 & dhe & CEM & 74 & 0.93 & - & 53.19 & $2.18 \times 10^{-7}$ & 0.127 & 70 & 97 & 70 & 68 & 1.079 & N117 & [212] \\
\hline 320 & SPI & dho & CEM & 150 & 0.40 & - & - & - & - & 100 & 98 & 73 & 72 & 1.220 & N117 & [213] \\
\hline
\end{tabular}


Due to the large number of data points, not every point in Figures 6-8 is marked with the sample number from the following Tables 5-14. The results pertaining water uptake, $\mathrm{D}_{\mathrm{r}}$ as well as the ion-exchange capacity $\left(\mathrm{IEC}_{\mathrm{A}}\right)$ for AEM and AIEM are presented as Supplementary Materials.

The Tables 5-14 list the membrane samples according to their membrane polymer structures, e.g., poly(sulfones). The tables start with the fluoro-carbon-based membranes followed by the hydro-carbon and the $N$-heterocycle-based membranes.

\subsection{Membrane Impact on VRFB Cell Performance}

A VRFB cell is built up with frames, electrode felts, bipolar plates, electrolyte and membranes. Figures 9-14 show the efficiencies of VRFB cells at different current densities. These cells are equipped with membranes from Tables 5-14.

Figure 9 shows the coulombic efficiencies $\left(\mathrm{CE}_{\mathrm{L}}\right)$ of VRFB cells with current densities of less than $100 \mathrm{~mA} \mathrm{~cm}^{-2}$, constructed with either fluoro-carbon, hydro-carbon or $N$ heterocycle-based samples.

$\mathrm{CE}_{\mathrm{L}}$ ranging from $65 \%$ to $99 \%$ were achieved with fluoro-carbon-based membrane samples, $\mathrm{CE}_{\mathrm{L}}$ from $66 \%$ to $100 \%$ with hydro-carbon-based membranes and $\mathrm{CE}_{\mathrm{L}}$ from $82 \%$ to $100 \%$ with $N$-heterocycle-based membranes.

In fluoro-carbons (Figure 9a), VRFB cells using MS95 (PTFE, dhe, CEM), MS133 (FPAE, dho, CEM), MS134 (FPAE, dho, CEM), MS132 (PFSA, dhe, CEM), MS129 (PFSA, dhe, CEM), MS126 (PFSA, dhe, CEM), MS122 (PFSA, dhe, AIEM) and MS107 (ETFE, dho, AIEM) achieve high $\mathrm{CE}_{\mathrm{L}}$ of at least $98 \%$.

In hydro-carbon-based membranes VRFB cells with modified PEEK membranes MS195 (PEEK, dho, AEM), MS161 (PEEK, dhe, CEM) and MS178 (PEEK, dhe, CEM) also achieve high $\mathrm{CE}_{\mathrm{L}}$ of at least 98\%. Furthermore, MS235 (PF, dho, AEM), MS254 (other, dho, AEM), MS195 (PSU, dho, AEM), MS218 (PPSU, dho, CEM), MS205 (PSU, sym, AEM) and MS225 (PES, asym, CEM) achieve similarly high $\mathrm{CE}_{\mathrm{L}}$. In Figure 9c VRFB cells with PBI membranes MS263 (PBI, dho, AEM*), MS264 (PBI, dho, AEM*), MS262 (PBI, dho, AEM*), MS272 (PBI, dho, AEM*), MS269 (PBI, asym, AEM*) and MS270 (PBI, sym, AEM*) show $\mathrm{CE}_{\mathrm{L}}$ of at least $98 \%$ as well. The VRFB cells with PPEK-based membranes (MS282-MS291) all show a CE $\mathrm{L}_{\mathrm{L}}$ of at least $98 \%$ except for MS292 (PPEK, dho, CEM).

VRFB cells equipped with PI membranes MS298 (PI, dho, CEM), MS301 (PI, dho, CEM), MS304 (PI, dho, AIEM*), MS305 (PI, dho, CEM) and MS308 (PI, dhe, AIEM*) all reach high $\mathrm{CE}_{\mathrm{L}}$ of at least $98 \%$.

In some cases, the $\mathrm{CE}_{\mathrm{L}}$ depend on the membrane thickness. This is observed with the PTFE-based membranes (Figure 9a). The $\mathrm{CE}_{\mathrm{L}}$ ranges from 94\% (MS92/25 $\mu \mathrm{m}$ ) to 99\% (MS96/70 $\mu \mathrm{m})$. The VRFB equipped with MS91 $(45 \mu \mathrm{m})$ shows a $\mathrm{CE}_{\mathrm{L}}$ of $96 \%$. Other examples do not show this correlation, e.g., MS111 with a thickness of $25 \mu \mathrm{m}$ and a $\mathrm{CE}_{\mathrm{L}}$ of $98 \%$ at $120 \mathrm{~mA} \mathrm{~cm}{ }^{2}$. In summary, it appears to be possible to reach high $\mathrm{CE}_{\mathrm{L}}$ with membrane polymers from all three groups.

Figure 10 shows the voltage efficiencies $\left(\mathrm{VE}_{\mathrm{L}}\right)$ of VRFB cells using the specified membrane samples with current densities less than $100 \mathrm{~mA} \mathrm{~cm}^{-2}$.

The $\mathrm{VE}_{\mathrm{L}}$ are $66 \%$ to $97.5 \%$ with fluoro-carbon-based membranes, $60.7 \%$ to $95 \%$ with hydro-carbon-based membranes and $65 \%$ to $96.4 \%$ with $N$-heterocycle-based membranes.

For the fluoro-carbon-based membranes in Figure $10 \mathrm{a}$, at a current density of $80 \mathrm{~mA} \mathrm{~cm}^{-2}$, the highest VE $\mathrm{L}_{\mathrm{L}}$ of VRFB cells are achieved using MS94 (PTFE, dhe, CEM), MS115 (PFSA, dhe, AIEM*), MS99 (PVDF, asym), MS119 (PFSA, dhe, CEM), MS98 (PVDF, asym), MS126 (PFSA, dhe, CEM) and MS91 (PTFE, dhe, CEM). The minimum $\mathrm{VE}_{\mathrm{L}}$ achieved here is $80 \%$.

For the hydro-carbon-based membranes in Figure 10b, at a current density of $80 \mathrm{~mA} \mathrm{~cm}^{-2}$, the highest VE $\mathrm{L}_{\mathrm{L}}$ of VRFB cells are obtained using MS229 (PES, asym, CEM), MS226 (PES, sym, AIEM), MS227 (PES, asym, AIEM), MS155 (PEEK, dho, CEM), MS154 (PEEK, dho, CEM), MS174 (PEEK, dhe, CEM), MS192 (PEEK, dho, CEM), MS167 (PEEK, dhe, CEM), MS176 (PEEK, dhe, CEM), MS214 (PPSU, dho, AEM), MS208 (PSU, asym, 
AIEM), MS194 (PEEK, dho, AEM) and MS249 (PF, dho, CEM). The minimum $\mathrm{VE}_{\mathrm{L}}$ achieved here is $85 \%$.
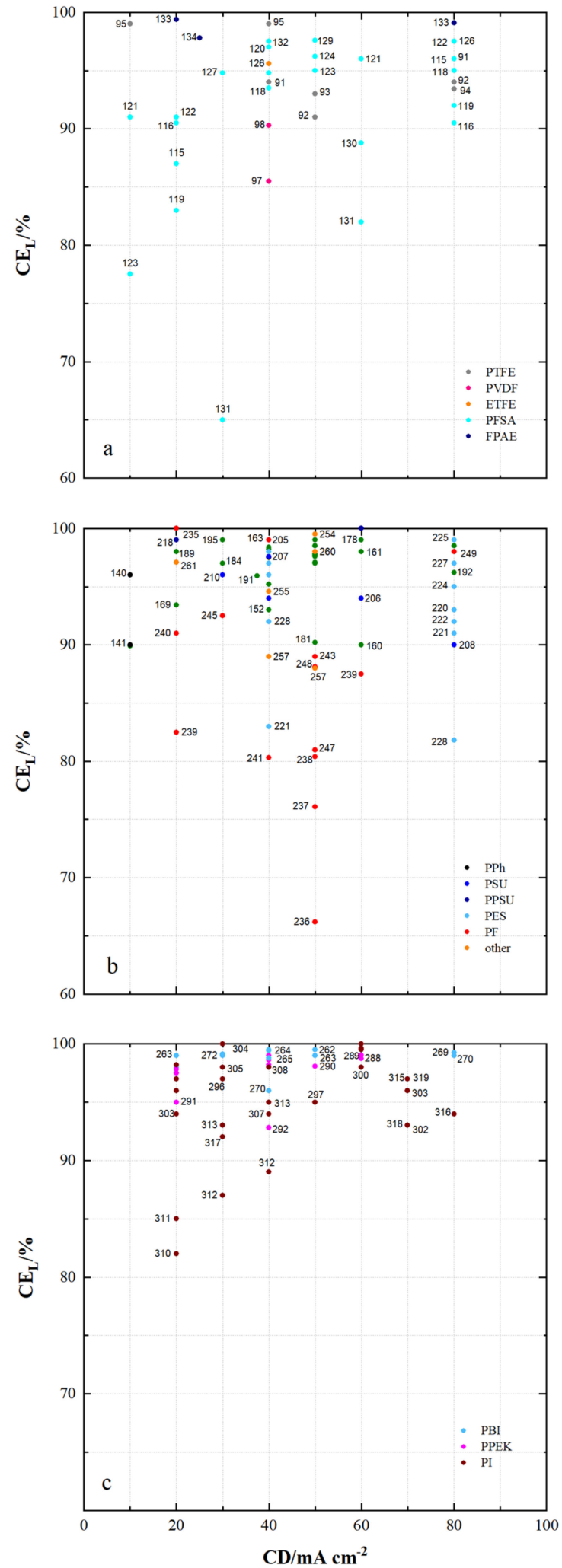

Figure 9. The energy efficiency of VRFB cells by using membrane samples at current densities $<100 \mathrm{~mA} \mathrm{~cm}^{-2}$ : (a) fluoro-carbons, (b) hydro-carbons and (c) $N$-heterocycles. 

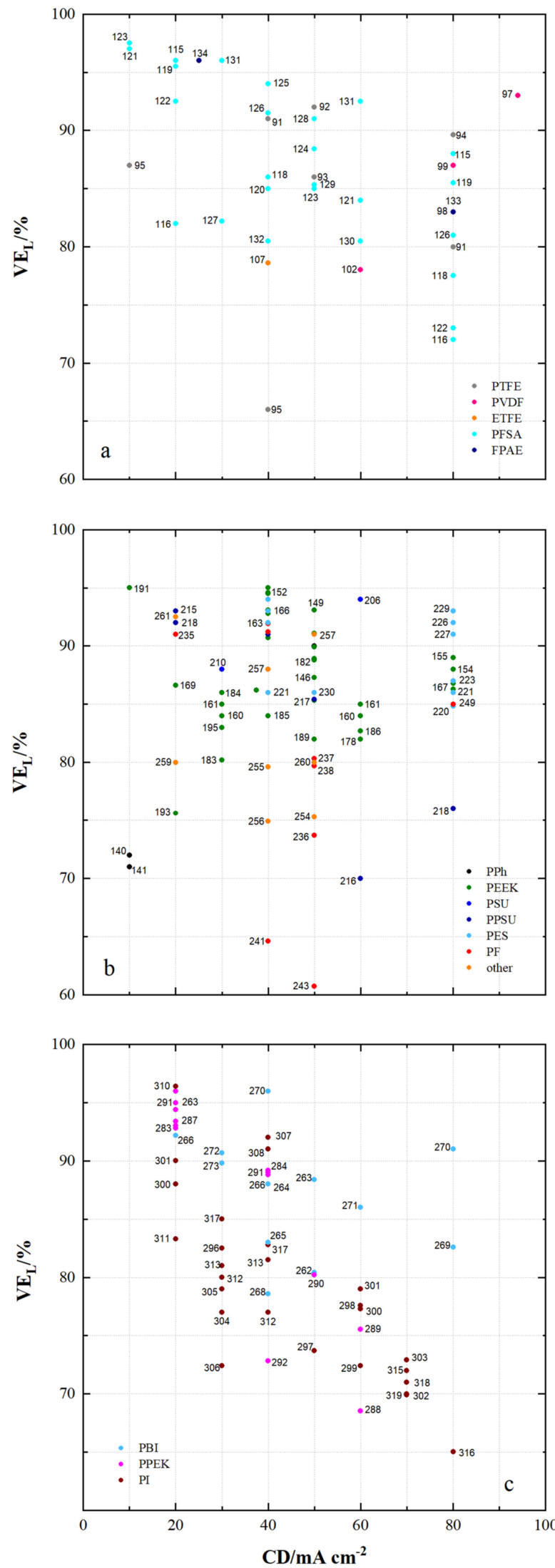

Figure 10. The voltage efficiency of VRFB cells by using membrane samples at current densities $<100 \mathrm{~mA} \mathrm{~cm}^{-2}$ : (a) fluoro-carbons, (b) hydro-carbons and (c) $N$-heterocycles. 

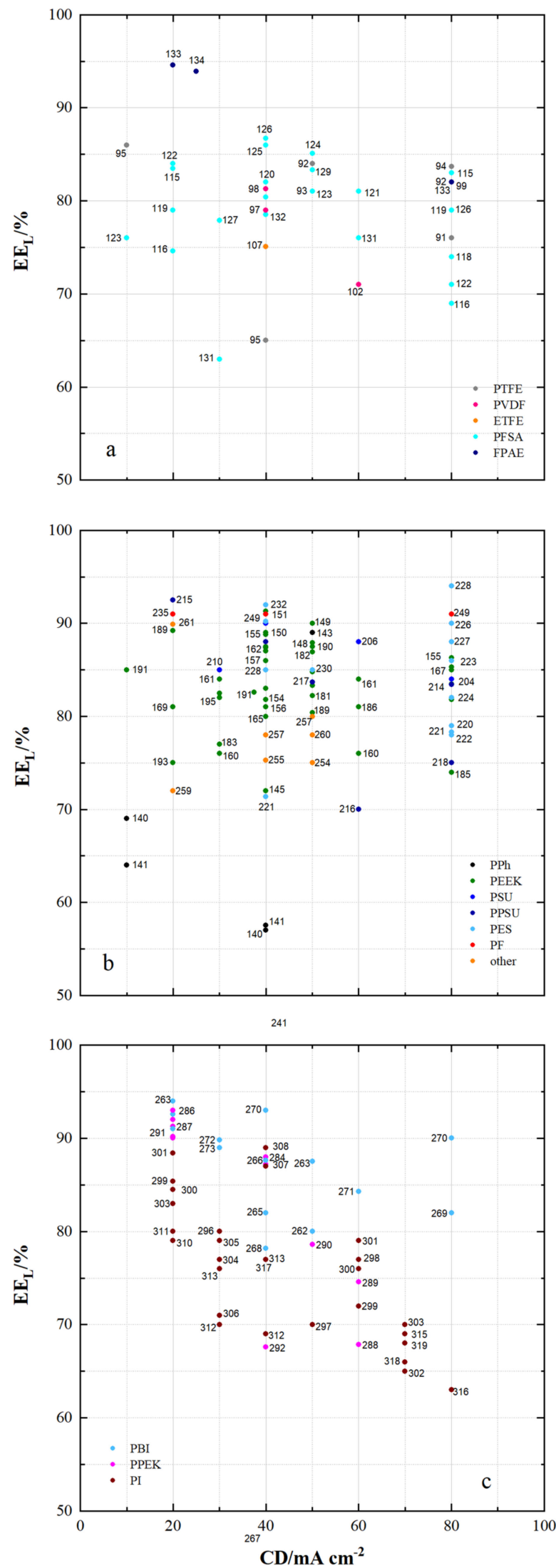

Figure 11. The energy efficiency of VRFB cells by using membrane samples at current densities $<100 \mathrm{~mA} \mathrm{~cm}^{-2}$ : (a) fluoro-carbons, (b) hydro-carbons and (c) $N$-heterocycles. 

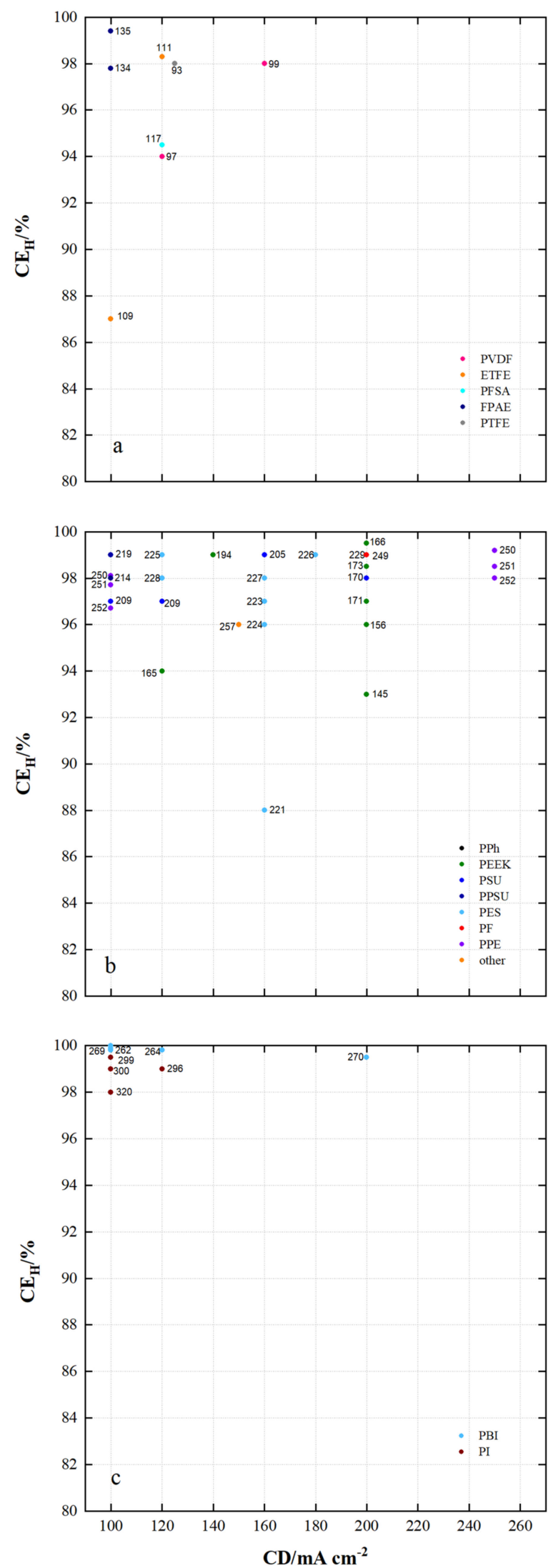

Figure 12. The coulombic efficiency of VRFB cells by using membrane samples at current densities $\geq 100 \mathrm{~mA} \mathrm{~cm}^{-2}$ : (a) fluoro-carbons, (b) hydro-carbons and (c) $N$-heterocycles. 

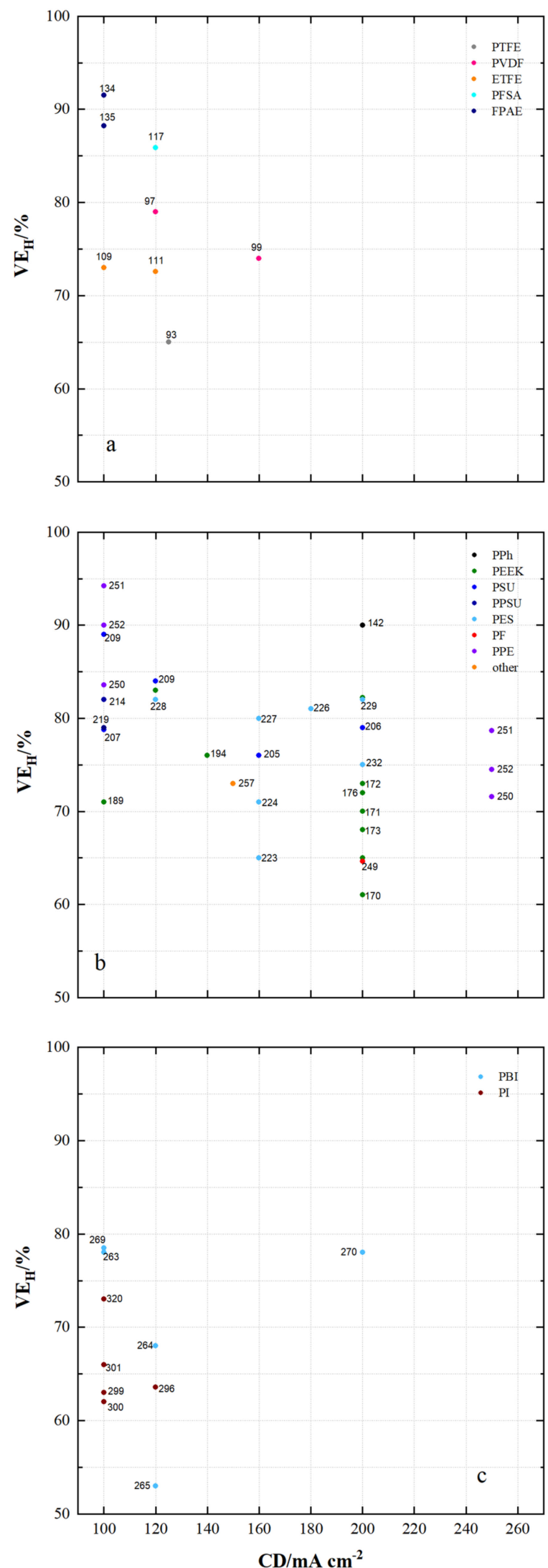

Figure 13. The voltage efficiency of VRFB cells by using membrane samples at current densities $\geq 100 \mathrm{~mA} \mathrm{~cm}^{-2}$ : (a) fluoro-carbons, (b) hydro-carbons and (c) $N$-heterocycles. 

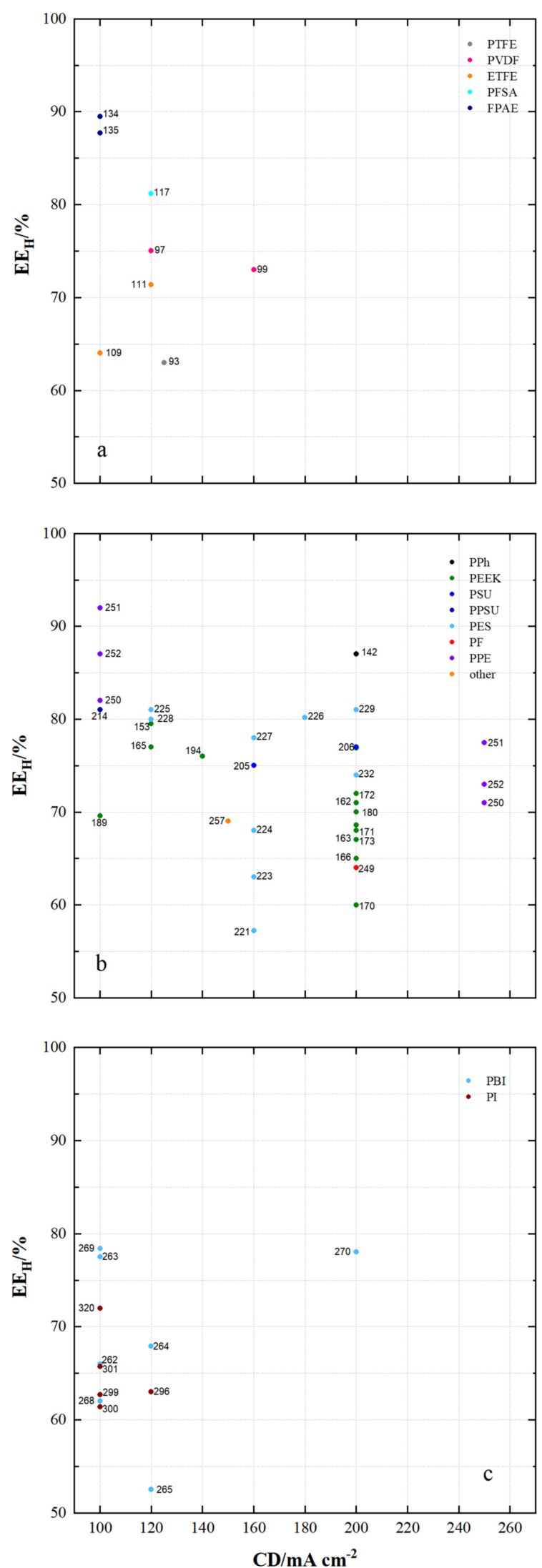

Figure 14. The energy efficiency of VRFB cells by using membrane samples at current densities $\geq 100 \mathrm{~mA} \mathrm{~cm}^{-2}$ : (a) fluoro-carbons, (b) hydro-carbons and (c) $N$-heterocycles. 
For the $N$-heterocycle-based membranes in Figure 10c, at a current density of $80 \mathrm{~mA} \mathrm{~cm}^{-2}$, the highest $\mathrm{VE}_{\mathrm{L}}$ of VRFB cells are achieved using MS270 (PBI, sym, AEM*) and MS269 (PBI, asym, AEM $^{*}$ ). All VE $\mathrm{L}_{\mathrm{L}}$ measured here are above $80 \%$.

$\mathrm{VE}_{\mathrm{L}}$ of at least $95 \%$ are reached by VRFB cells at lower current densities with MS123 (PFSA, dhe, AIEM), MS121 (PFSA, dhe, CEM), MS115 (PFSA, dhe, AIEM*), MS119 (PFSA, dhe, CEM),

MS134 (FPAE, dho, CEM), MS131 (PFSA, dhe, AIEM*), MS191 (PEEK, dhe, CEM), MS152 (PEEK, dho, CEM), MS310 (PI, dhe, CEM), MS270 (PBI, sym, AEM*), MS263 (PBI, dho, AEM $^{*}$ ) and MS291 (PPEK, dho, CEM).

Figure 10a,c show the tendency of decreasing $\mathrm{VE}_{\mathrm{L}}$ with increasing current density, which cannot be observed as a trend for hydro-carbon-based membranes. This can be explained by a lower electrical resistance, which can be achieved with aromatic polymers with high degrees of sulfonation.

Figure 11 shows the energy efficiency $\left(\mathrm{EE}_{\mathrm{L}}\right)$ of VRFB cells using the specified membrane samples with current densities less than $100 \mathrm{~mA} \mathrm{~cm}^{-2}$.

For VRFB cells with fluoro-carbon-based membrane samples, the $\mathrm{EE}_{\mathrm{L}}$ ranges from $63 \%$ to $95 \%$. For hydro-carbon-based membranes the $\mathrm{EE}_{\mathrm{L}}$ ranges from $57 \%$ to $94 \%$ and for N-heterocycle-based membranes the $\mathrm{EE}_{\mathrm{L}}$ ranges from $63 \%$ to $94 \%$.

For VRFB cells with fluoro-carbon-based membranes, high $\mathrm{EE}_{\mathrm{L}}$ of at least $85 \%$ are measured with MS133 (FPAE, dho, CEM), MS134 (FPAE, dho, CEM), MS126 (PFSA, dhe, CEM), MS125 (PFSA, dhe, CEM), MS95 (PTFE, dhe, CEM) and MS124 (PFSA, dhe, AIEM*).

For VRFB cells with hydro-carbon-based membranes, $\mathrm{EE}_{\mathrm{L}}$ of at least $90 \%$ are achieved with MS215 (PPSU, dho, AEM), MS235 (PF, dho, AEM), MS232 (FPAE, dhe, CEM), MS151 (PEEK, dhe, CEM), MS249 (PF, dho, CEM), MS149 (PEEK, dho, CEM) and MS228 (PES, dhe, CEM). With many other membranes from Figure $11 \mathrm{~b}, \mathrm{EE}_{\mathrm{L}}$ of at least $85 \%$ were achieved.

In VRFB cells with $N$-heterocycle-based membranes, high $\mathrm{EE}_{\mathrm{L}}$ of at least $85 \%$ are measured with MS263 (PBI, dho, AEM*), MS286 (PPEK, dho, AEM), MS287 (PPEK, dho, AEM), MS291 (PPEK, dho, CEM), MS301 (PI, dho, CEM), MS299 (PI, dho, CEM), MS272 (PBI, dho, AIEM*), MS273 (PBI, dho, AIEM*), MS308 (PI, dhe, AIEM*), MS284 (PPEK, dho, AEM), MS266 (PBI, sym, AEM*), MS307 (PI, dho, CEM), MS263 (PBI, dho, AEM*) and MS270 (PBI, sym, AEM*).

The improvement of the $\mathrm{EE}_{\mathrm{L}}$ e.g., at $80 \mathrm{~mA} \mathrm{~cm}^{-2}$ is caused by the optimization of different membrane properties. The series of SFPAE membranes $(28,45,80 \mu \mathrm{m})$ in [114] showed a different energy efficiency due to different membrane thickness. A water uptake of 30\% leads to the highest energy efficiency of $90 \%$ at $50 \mathrm{~mA} \mathrm{~cm}^{-2}$ by using PEEK-based CEM.

It has been shown that with a range of different membrane samples, high $\mathrm{EE}_{\mathrm{L}}$ of over $90 \%$ at current densities of less than $100 \mathrm{~mA} \mathrm{~cm}^{-2}$ are feasible. These high efficiencies are achieved by cells with dense fluoro-carbon and dense hydro-carbon-based membranes as well as dense and symmetrically porous $N$-heterocycle-based membranes. The respective membranes are composed of different polymers and can be assigned to CEM, AEM, AEM* and AIEM*.

Figure 12 shows the coulomb efficiencies $\left(\mathrm{CE}_{\mathrm{H}}\right)$ of VRFB cells with current densities of at least $100 \mathrm{~mA} \mathrm{~cm}^{-2}$ using the specified membrane samples.

In the VRFB cells from Figure 12a equipped with the fluoro-carbon-based membrane samples, $\mathrm{CE}_{\mathrm{H}}$ of $87 \%$ to $99.5 \%$ were measured. The VRFB cells equipped with the hydrocarbon-based membranes shown in Figure $12 \mathrm{~b}$ achieved $\mathrm{CE}_{\mathrm{H}}$ from $88 \%$ to $99.5 \%$. Using the $N$-heterocycle-based membranes shown in Figure $12 \mathrm{c}, \mathrm{CE}_{\mathrm{H}}$ of $98 \%$ to $99.8 \%$ were realized.

In fluoro-carbon-based membranes VRFB cells with MS135 (FPAE, dhe, CEM), MS111 (ETFE, dho, AIEM), MS93 (PTFE, sym, -) and MS97 (PVDF, sym, -) also achieve high CE $_{\mathrm{H}}$ of at least $98 \%$.

In hydro-carbon-based membranes VRFB cells with MS219 (PPSU, dhe, AIEM*), MS214 (PPSU, dho, AEM), MS250 (PPE, dho, AEM), MS225 (PES, asym, CEM), MS228 (PES, dhe, CEM), MS194 (PEEK, dho, AEM), MS227 (PES, asym, AIEM*), MS205 (PSU, sym, AEM), MS226 (PES, sym, AIEM), MS166 (PEEK, dho, CEM), MS229 (PES, asym, CEM), 
MS249 (PF, dho, CEM), MS173 (PEEK, dhe, CEM), MS170 (PEEK, dho, CEM), MS251 (PPE, dho, AEM) and MS252 (PPE, dho, AEM) reach high $\mathrm{CE}_{\mathrm{H}}$ of at least $98 \%$.

For the $N$-heterocycle-based membranes all VRFB cells shown reach $\mathrm{CE}_{\mathrm{H}}$ of at least 98\%. These include MS269 (PBI, asym, AEM*), MS262 (PBI, dho, AEM*), MS264 (PBI, dho, AEM $^{*}$ ), MS299 (PI, dho, CEM), MS300 (PI, dho, CEM), MS320 (PI, dho, CEM), MS296 (PI, dho, CEM) and MS270 (PBI, sym, AEM*).

Low $\mathrm{CE}_{\mathrm{H}}$ were measured with MS109 (ETFE, dho, CEM), MS145 (PEEK, dho, CEM) and MS221 (PES, sym, CEM). It can be assumed that the water uptake of 181\% for MS109 and $88 \%$ for MS145 as well as large pores for MS221 during cycling led to excessive electrolyte transfer and thus to charge loss.

Lower $D_{c}$ measured for the membranes in Figure 12 enable higher coulombic efficiencies. For example, in contrast to MS145 $\left(\mathrm{D}_{\mathrm{c}}=3.06 \times 10^{-6} / \mathrm{CE}_{\mathrm{H}}=93 \%\right)$ higher $\mathrm{CE}_{\mathrm{H}}$ are realized with MS162 $\left(D_{c}=1.04 \times 10^{-7} / \mathrm{CE}_{\mathrm{H}}=97 \%\right), \operatorname{MS163}\left(\mathrm{D}_{\mathrm{c}}=1.67 \times 10^{-7} / \mathrm{CE}_{\mathrm{H}}=99 \%\right)$ and MS173 $\left(D_{c}=3.5 \times 10^{-7} / \mathrm{CE}_{\mathrm{H}}=99 \%\right)$ at the same current density of $200 \mathrm{~mA} \mathrm{~cm}{ }^{2}$.

Figure 13 shows the voltage efficiencies $\left(\mathrm{VE}_{\mathrm{H}}\right)$ of VRFB cells with current densities of at least $100 \mathrm{~mA} \mathrm{~cm}^{-2}$ using the specified membrane samples. The $\mathrm{VE}_{\mathrm{H}}$ of VRFB cells using the fluorocarbon-based membrane samples shown in Figure 13a ranges from $65 \%$ to $92 \%$.

$\mathrm{VE}_{\mathrm{H}}$ of $61 \%$ to $99 \%$ are achieved using the hydro-carbon-based membranes shown in Figure $12 \mathrm{~b}$ and $\mathrm{VE}_{\mathrm{H}}$ of $53 \%$ to $78 \%$ using the $N$-heterocycle-based membrane samples shown in Figure 12c.

High $\mathrm{VE}_{\mathrm{H}}$ of at least $80 \%$ are measured with MS134 (FPAE, dho, CEM), MS135 (FPAE, dhe, CEM), MS117 (PFSA, dhe, CEM), MS251 (PPE, dho, AEM), MS252 (PPE, dho, AEM), MS209 (PSU, sym, AEM), MS142 (DAPP, dho, AEM), MS250 (PPE, dho, AEM), MS214 (PPSU, dho, AEM), MS209 (PSU, sym, AEM), MS228 (PES, dhe, CEM), MS164 (PEEK, dho, CEM), MS225 (PES, asym, CEM), MS226 (PES, sym, AIEM), MS229 (PES, asym, CEM) and MS145 (PEEK, dho, CEM).

For VRFBs equipped with $N$-heterocycle-based membranes, the highest $\mathrm{VE}_{\mathrm{H}}$ with MS269 (PBI, asym, AEM*) is 78.5\%, with MS263 (PBI, dho, $\mathrm{AEM}^{*}$ ) and with MS270 (PBI, sym, $\left.\mathrm{AEM}^{*}\right) 78 \%$.

With the exception of MS270 in Figure 13c, the results in Figure 13a-c show the tendency of the $\mathrm{VE}_{\mathrm{H}}$ to decrease with increasing current density.

Figure 14 shows the energy efficiency $\left(\mathrm{EE}_{\mathrm{H}}\right)$ of VRFB cells with a current density of at least $100 \mathrm{~mA} \mathrm{~cm}^{-2}$ using the specified membrane sample.

Energy efficiencies of $63 \%$ to $89.5 \%$ were achieved with fluoro-carbon-based membranes, $57.2 \%$ to $92 \%$ with hydro-carbon-based membranes and $52.5 \%$ to $78.4 \%$ with $\mathrm{N}$-heterocycle-based membranes.

Using fluoro-carbon-based membranes, VRFB cells with MS134 (FPAE, dho, CEM) achieve an $\mathrm{EE}_{\mathrm{H}}$ of $89.5 \%$ at a current density of $100 \mathrm{~mA} \mathrm{~cm}^{-2}$, with MS135 (FPAE, dhe, CEM) an $\mathrm{EE}_{\mathrm{H}}$ of $87.7 \%$ at a current density of $100 \mathrm{~mA} \mathrm{~cm}^{-2}$ and with MS117 (PFSA, dhe, $\mathrm{CEM}$ ) an $\mathrm{EE}_{\mathrm{H}}$ of $81 \%$ at a current density of $120 \mathrm{~mA} \mathrm{~cm}^{-2}$ at a current density of $100 \mathrm{~mA} \mathrm{~cm}^{-2}$.

Using hydro-carbon-based membranes, VRFB cells can be obtained with MS251 (PPE, dho, AEM), MS252 (PPE, dho, AEM), MS250 (PPE, dho, AEM), MS214 (PPSU, dho, AEM), MS225 (PES, asym, CEM), MS228 (PES, dhe, CEM), MS226 (PES, sym, AIEM), MS229 (PES, asym, CEM) and MS142 (DAPP, dho, AEM) and have an $\mathrm{EE}_{\mathrm{H}}$ of at least $80 \%$.

In VRFB cells with N-heterocycle-based membranes, the highest $\mathrm{EE}_{\mathrm{H}}$ are between $70 \%$ and $80 \%$. MS269 (PBI, asym, AEM*), MS263 (PBI, dho, AEM*), MS270 (PBI, sym, AEM*) and MS320 (PI, dho, CEM) were used.

Figure 15 shows the $E_{\mathrm{r}}$ (calculated with the reference membrane from Tables 5-14) of VRFB cells and the respective publication year. 

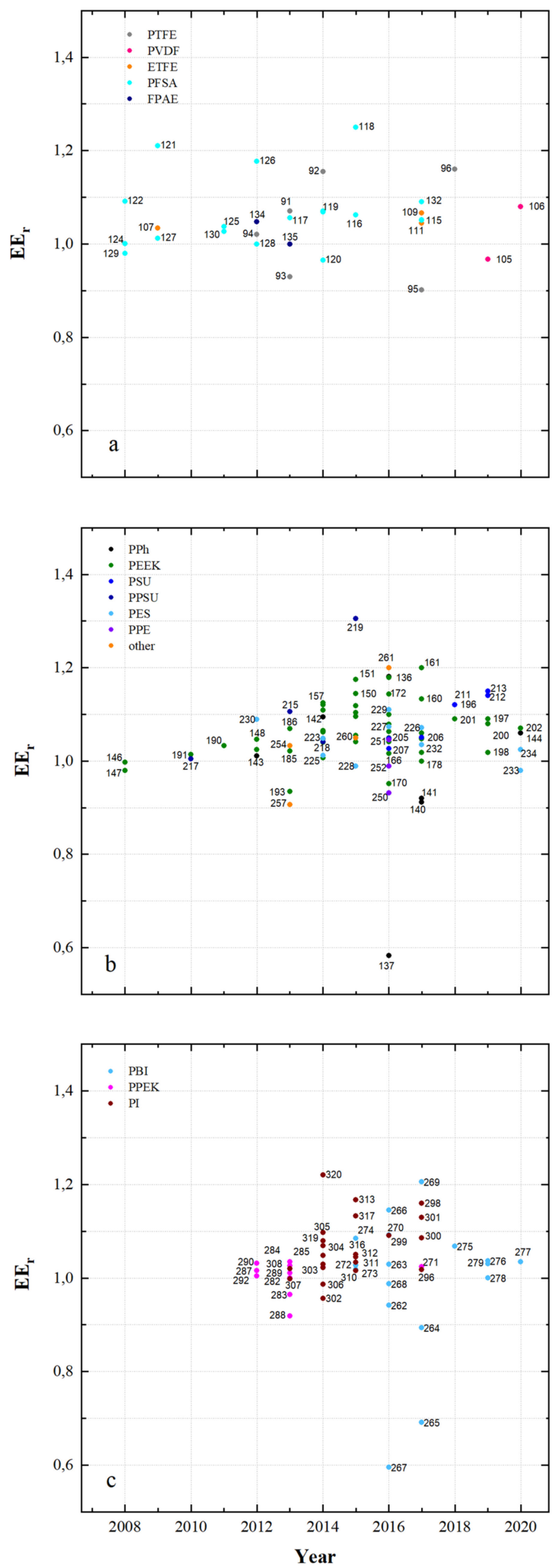

Figure 15. Energy efficiency ratios in recent years: (a) fluoro-carbons, (b) hydro-carbons and (c) $\mathrm{N}$-heterocycles. 
An $E_{\mathrm{r}}$ of 0.901 to 1.250 is obtained with fluoro-carbon-based membranes, an $E_{\mathrm{r}}$ of 0.907 to 1.305 is obtained with hydro-carbon-based membranes and an $E_{\mathrm{r}}$ of 0.919 to 1.220 is obtained with $N$-heterocycle-based membranes.

With MS118 (PFSA, dhe, CEM), MS121 (PFSA, dhe, CEM), MS126 (PFSA, dhe, CEM) and MS92 (PTFE, dhe, CEM), an $E_{\mathrm{r}}$ of at least 1.1 was achieved for the fluorocarbon-based membranes. The hydro-carbon-based membranes were tested with MS219 (PPSU, dho, AIEM*), MS161 (PEEK, dhe, CEM), MS136 (DAPP, dho, CEM), MS151 (PEEK, dhe, CEM), MS150 (PEEK, dho, CEM), MS172 (PEEK, dhe, CEM), MS160 (PEEK, dho, CEM) and MS157 (PEEK, dho, CEM) and achieved a high $\mathrm{EE}_{\mathrm{r}}$ of at least 1,1. Among others, MS320 (PI, dho, CEM), MS269 (PBI, asym, AEM*), MS313 (PI, dhe, AIEM*), MS266 (PBI, sym, AEM*), MS298 (PI, dho, CEM), MS301 (PI, dho, CEM) and MS317 (PI, dhe, AIEM*) achieved a high $\mathrm{EE}_{\mathrm{r}}$ of at least 1.1.

Potential for improvement of the VRFB can be seen especially in the hydro-carbon and $N$-heterocycle-based membranes in Figure 15b,c. This improvement represented by $\mathrm{EE}_{\mathrm{r}}$ is also observed with the fluoro-carbons, however, in weaker expression.

In conclusion, Figure 15 and Table 15 both show that membrane change often leads to improved energy efficiency under otherwise identical test conditions.

Table 15. High energy efficiency ratios.

\begin{tabular}{ccccccccc}
\hline \multirow{2}{*}{$\mathbf{M S}$} & \multirow{2}{*}{$\begin{array}{c}\text { Polymer } \\
\text { Used }\end{array}$} & $\mathbf{E E}_{\mathbf{r}}$ & $\mathbf{C D}$ & $\mathbf{D}_{\mathbf{r}}$ & $\mathbf{d}$ & $\mathbf{W U}$ & $\mathbf{I E C}$ & \multirow{2}{*}{ Ref. } \\
\cline { 3 - 7 } 219 & PPSU & 1.305 & 100 & - & 50 & - & 1.2 & {$[34]$} \\
118 & PFSA & 1.250 & 80 & 0.5 & - & 31 & 0.925 & {$[112]$} \\
320 & PI & 1.220 & 100 & - & 150 & - & 0.4 & {$[213]$} \\
121 & PFSA & 1.210 & 80 & 0.05 & 217 & 23.6 & 0.97 & {$[131]$} \\
269 & PBI & 1.206 & 80 & - & - & - & - & {$[194]$} \\
261 & other & 1.200 & 20 & - & - & - & - & {$[191]$} \\
161 & SPEEK & 1.200 & 60 & 0.061 & 80 & 20.7 & 0.86 & {$[72]$} \\
136 & SDAPP & 1.181 & 200 & - & 41 & - & 1.8 & {$[139]$} \\
139 & qDAPP & 1.181 & 200 & - & 54 & - & 1.2 & {$[139]$} \\
184 & SPEEK & 1.179 & 30 & 0.179 & - & 22 & 1.08 & {$[147]$} \\
126 & PFSA & 1.177 & 70 & - & 193 & 14.3 & 0.92 & {$[134]$} \\
151 & SPEEK & 1.175 & 40 & 0.168 & 37 & 21 & 1.44 & {$[33]$} \\
313 & PI & 1.167 & 40 & 0.104 & 50 & 44.2 & 1.16 & {$[210]$} \\
298 & PI & 1.160 & 60 & 0.055 & - & 17.8 & 1.5 & {$[207]$} \\
92 & PTFE & 1.155 & 80 & 0.45 & 25 & 65.5 & - & {$[115]$} \\
\hline
\end{tabular}

Publications show, in part, the influence of the polymer and membrane properties on VRFB cell performance. Water uptake and the degree of functionalization can be optimized by the use of cross-linkers [35]. An optimum of 18\% (WU) is determined for the PPE-based membranes (dho, AEM) [35]. At this optimum and a current density of $100 \mathrm{~mA} \mathrm{~cm}^{-2}$ the maximum CE, VE and EE is $97.7 \%, 94 \%$ and $92 \%$. Here, an EE $E_{r}$ of 1.045 compared to N212 can be achieved. Permeability can be improved by introducing positive charges into the polymer [162]. This leads to the ability of the membrane to keep CE high and self-discharge of a VRFB cell low.

An important property of polymer membranes is the ion-selectivity which can be determined by proton conductivity and permeation experiments. This selectivity can further be optimized by adjusting the thickness of CEM [113] to maximize the CE, VE and EE of the VRFB cell.

Ionically cross-linked blend membranes [34] represent one of the well-balanced compromises regarding these properties. The ionic cross-linking of PBI and sulfonated PPSU enables reduced water uptake combined with comparatively high $\mathrm{IEC}_{\mathrm{c}}$ and high proton conductivity. This type of membrane with a thickness of $50 \mu \mathrm{m}$ enables a high EE of 77\% and an $\mathrm{EE}_{\mathrm{r}}$ of 1305 compared to $\mathrm{N} 117$ at $100 \mathrm{~mA} \mathrm{~cm}^{-2}$. 
Good results can also be achieved with PBI membranes containing enhanced targeted porous structures. Using symmetric porous structures, PBI membranes from [193] and [195] enable energy efficiencies of $87 \%$ and $90 \%$. The asymmetrically porous PBI membrane from [194] enables a comparatively high energy efficiency of $82 \%$ as well.

Furthermore, it is possible to design porous membranes with neutral polymers such as PVDF (MS99, asym) or PTFE (MS93, sym). These appear to have improved long-term stability [91,118].

While Tables 5-14 provide an exhaustive list of $E_{\mathrm{r}}$ values, Table 15, for the sake of brevity, contains only the fifteen flow-battery targeted membrane samples which showed the highest values during our investigation.

\section{Cycle Stability}

Various methods are used to evaluate the stability of membranes. It is possible to determine the weight loss over time at a certain temperature by way of mass balance. This is done using swelling tests in aggressive media such as Fenton's reagent or charged VRFB catholytes. Another method is VRFB cycling tests, which plot the achieved battery performance over a number of cycles graphically. This test is performed to evaluate the stability of the membrane for a given number of cycles [144] or to determine the time of failure of the membrane [206].

Table 16 shows results of VRFB's cycling tests. In most cases, a current density of 40-80 $\mathrm{mA} \mathrm{cm}^{-2}$ was used to show cycle stability. Cycle stability at current densities of 120-200 mA cm ${ }^{-2}$ was demonstrated in some cases [35,45,74,103,134,199]. Furthermore, the electrolyte quantities in these cycling tests varied. A comparatively high cycle stability of 13000 cycles was demonstrated with a symmetrically porous PBI membrane [195]. A total of 6000 cycles were achieved with a symmetrically porous PSU membrane (AEM, crosslinked) [102]. 4000 charge/discharge cycles were performed with a partially fluorinated and vinylimidazole-based AEM [41]. 1000 cycles were completed with an asymmetrically porous PVDF membrane [118] and a sPEEK-based cation exchange membrane [144]. Many other results with cycles between 50 and 13,000 can be found in Table 16 .

Table 16. Cycle stability of published membrane samples.

\begin{tabular}{|c|c|c|c|c|c|c|c|c|c|}
\hline Polymer & MS & Cycles & $\mathrm{mL} \mathrm{I} \mathrm{mA} \mathrm{cm}^{-2}$ & Ref. & Polymer & MS & Cycles & $\mathrm{mL} \mathrm{I} \mathrm{mA} \mathrm{cm}^{-2}$ & Ref. \\
\hline PTFE & 94 & 700 & -180 & [88] & \multirow{2}{*}{ PES } & 224 & 70 & $40 \mid 80$ & [174] \\
\hline \multirow{4}{*}{ PVDF } & 97 & 50 & $30 \mid 80$ & [117] & & 230 & 100 & $80 \mid 50$ & [176] \\
\hline & 98 & 1000 & $30 \mid 80$ & [118] & PPE & 251 & 500 & $25 \mid 200$ & [35] \\
\hline & 99 & 300 & $30 \mid 80$ & [119] & \multirow{4}{*}{ other } & 254 & 4000 & -150 & [41] \\
\hline & 102 & 230 & $40 \mid 60$ & [68] & & 255 & 150 & $3 \mid 40$ & [186] \\
\hline \multirow{2}{*}{ PFSA } & 116 & 150 & $60 \mid 80$ & [64] & & 256 & 500 & $3 \mid 40$ & [187] \\
\hline & 117 & 300 & $-\mid 120$ & [130] & & 259 & 120 & $3 \mid 20$ & [190] \\
\hline DAPP & 143 & 400 & $100 \mid 50$ & [43] & \multirow{4}{*}{ PBI } & 262 & 200 & $100 \mid 50$ & [65] \\
\hline \multirow{8}{*}{ PEEK } & 155 & 120 & $50 \mid 60$ & [58] & & 270 & 13000 & $60 \mid 80-120$ & [195] \\
\hline & 157 & 100 & $50 \mid 80$ & [60] & & 271 & 300 & $10 \mid 60$ & [87] \\
\hline & 161 & 300 & $10 \mid 30$ & [72] & & 272 & 220 & $20 \mid 30$ & [63] \\
\hline & 167 & 1000 & -180 & [144] & \multirow{2}{*}{ PPEK } & 290 & 100 & $120 \mid 50$ & [79] \\
\hline & 174 & 180 & $30 \mid 80$ & [59] & & 291 & 100 & $30 \mid 60$ & [61] \\
\hline & 176 & 500 & $50 \mid 80$ & [145] & \multirow{3}{*}{ PI } & 296 & 500 & $30 \mid 30-120$ & [74] \\
\hline & 180 & 50 & $50 \mid 60$ & [38] & & 297 & 750 & $30 \mid 50$ & [206] \\
\hline & 194 & 100 & $30 \mid 80$ & [98] & & 298 & 100 & -160 & [207] \\
\hline
\end{tabular}


Table 16. Cont.

\begin{tabular}{|c|c|c|c|c|c|c|c|c|c|}
\hline Polymer & MS & Cycles & $\mathrm{mL} / \mathrm{mA} \mathrm{cm}{ }^{-2}$ & Ref. & Polymer & MS & Cycles & $\mathrm{mL} / \mathrm{mA} \mathrm{cm}^{-2}$ & Ref. \\
\hline \multirow{4}{*}{ PSU } & 204 & 900 & -180 & [162] & & 301 & 100 & $60 \mid 60$ & [78] \\
\hline & 205 & 6000 & $100 \mid 120$ & [102] & & 311 & 500 & $30 \mid 25-70$ & [209] \\
\hline & 207 & 500 & $50 \mid 80$ & [163] & & 313 & 100 & $8 \mid 40$ & [210] \\
\hline & 208 & 300 & $30 \mid 80$ & [164] & & 317 & 100 & $8 \mid 40$ & [211] \\
\hline \multirow{2}{*}{ PES } & 220 & 150 & $30 \mid 80$ & [171] & \multirow{2}{*}{ Nafion } & 32 & 200 & $50 \mid 160$ & {$[45]$} \\
\hline & 221 & 250 & $60 \mid 80$ & [172] & & 56 & 200 & $50 \mid 160$ & [45] \\
\hline
\end{tabular}

\section{Membrane Costs}

The cost of a VRFB varies with its electrical power (stack size) and available storage capacity (volume of battery electrolyte). The cost proportion of the installed components can therefore vary greatly. A cost analysis conducted by the U.S. Department of Energy (DOE) showed that the cost proportion of the membrane, measured against the total system, is $44 \%$ for a plant with a storage capacity of $0.25 \mathrm{MWh}$ and $27 \%$ for a plant with a storage capacity of $4 \mathrm{MWh}$ [35]. The cost proportion of the system is stated to be even lower at 10-15\% in [65]. In relation to the stack costs, however, a cost proportion of about $40 \%$ has been assumed for the use of Nafion [52,65,86,92,185,193,197], whereby also cost ranges of $30-50 \%$ of the stack were mentioned [144]. The high specific cost of Nafion (500-800 USD m ${ }^{-2}$ ) [86,189] is reported disparately, since with decreasing mass per square meter and different membrane thickness $(50.8 \mu \mathrm{m}$ to $183 \mu \mathrm{m})$ as well as different purchase quantities, the price varies. In [46] is mentioned that a quantity of $0.3 \times 20 \mathrm{~m}^{2} \mathrm{~N} 212$ is about $50 \%$ cheaper than N115. In [163] N212 is quoted at 225 USD m$^{-2}$. Referring to [46] this results in a cost saving of $12 \%$ to $25 \%$ if N212 is preferred to a N115 membrane. Substituting the N115 membrane for a Vanadion membrane reduces the cost from $331 \mathrm{USD} \mathrm{kWh}^{-1}$ to $251 \mathrm{USD} \mathrm{kWh}^{-1}$ for a $1 \mathrm{MWh}$ plant [27]. Furthermore, rough cost estimates are given for published flow-battery targeted membranes. It is assumed that a PES-based membrane is about 1/10 of the price of N115 [174]. PEEK are generally said to have lower production costs due to the aromatic main chain [33]. For PPSU-based membranes, the price could be about $1 / 400$ of Nafion [48]. For partially fluorinated sulfonated PI membranes the manufacturing cost is 167 USD m $^{-2}$ [206]. With material costs of 100 USD m$^{-2}$, the cost of PAEK membranes appears to be lower than the cost of Nafion [152]. The cost for PSU membranes is 21 to 24 USD m$^{-2}$ [163]. Further, when using PSU membranes, a cost saving of 1/20 compared to Nafion is reported [165]. Table 17 gives an overview of low-cost flow-battery targeted membranes in recent years.

Table 17. Published low-cost membranes.

\begin{tabular}{cccccc}
\hline MS & Membrane Polymer & Ref. & MS & Membrane Polymer & Ref. \\
\hline 224 & PES & {$[174]$} & 129 & PFSA & {$[136]$} \\
151 & PEEK & {$[33]$} & 167 & PEEK/PTFE & {$[144]$} \\
263 & PBI & {$[65]$} & 93 & PTFE & {$[91]$} \\
191 & PEEK & {$[150]$} & 98 & PVDF & {$[118]$} \\
217 & PPSU & {$[48]$} & 251 & PPE & {$[35]$} \\
297 & PI & {$[206]$} & 207 & PSU & {$[163]$} \\
266 & PBI & {$[193]$} & 286 & PPEK & {$[204]$} \\
243 & PF & {$[181]$} & 209 & PSU & {$[165]$} \\
258 & Other & {$[189]$} & 10 & PFSA & {$[27]$} \\
\hline
\end{tabular}

C. Minke et al. have dealt with the costs of VRFB, in particular the costs of membranes more extensively. This is how a cost proportion of $37 \%$, for a $250 \mathrm{~kW}$ stack using Nafion membrane, is calculated [214]. The use of sPEEK membranes could reduce the cost propor- 
tion to $8 \%$. This would reduce the cost of a $250 \mathrm{~kW}$ stack from 219,000 EUR to 150,000 EUR. They also describe that the specific price of membranes depends on the production volume. A calculation in [215] shows that the price of Nafion can be reduced from $300 \mathrm{USD} \mathrm{m}^{-2}$ to approximately $20 \mathrm{USD} \mathrm{m}^{-2}$ if the production quantity is increased from 0.01 to 10 million square meters per year. The comprehensive listing of membrane costs in [10] describes a current cost range of 16-451 EUR $\mathrm{m}^{-2}$.

Looking at the raw material prices in the plastics industry, differences can be seen in the specific costs for polymer granulates, which are used in various flow-battery targeted membranes. The Cambridge Engineering Selector database [216] provides an overview (Figure 16). PS and PP are traded at significantly less than $5 \mathrm{EUR} \mathrm{kg}^{-1}$. PSU, PES, ETFE, PVDF, PTFE and PPSU are traded in the range from 10 to 12 EUR kg-1. The high-performance polymers PEEK, PEK, PEKK and PI range from 70 to $110 \mathrm{EUR} \mathrm{kg}^{-1}$.

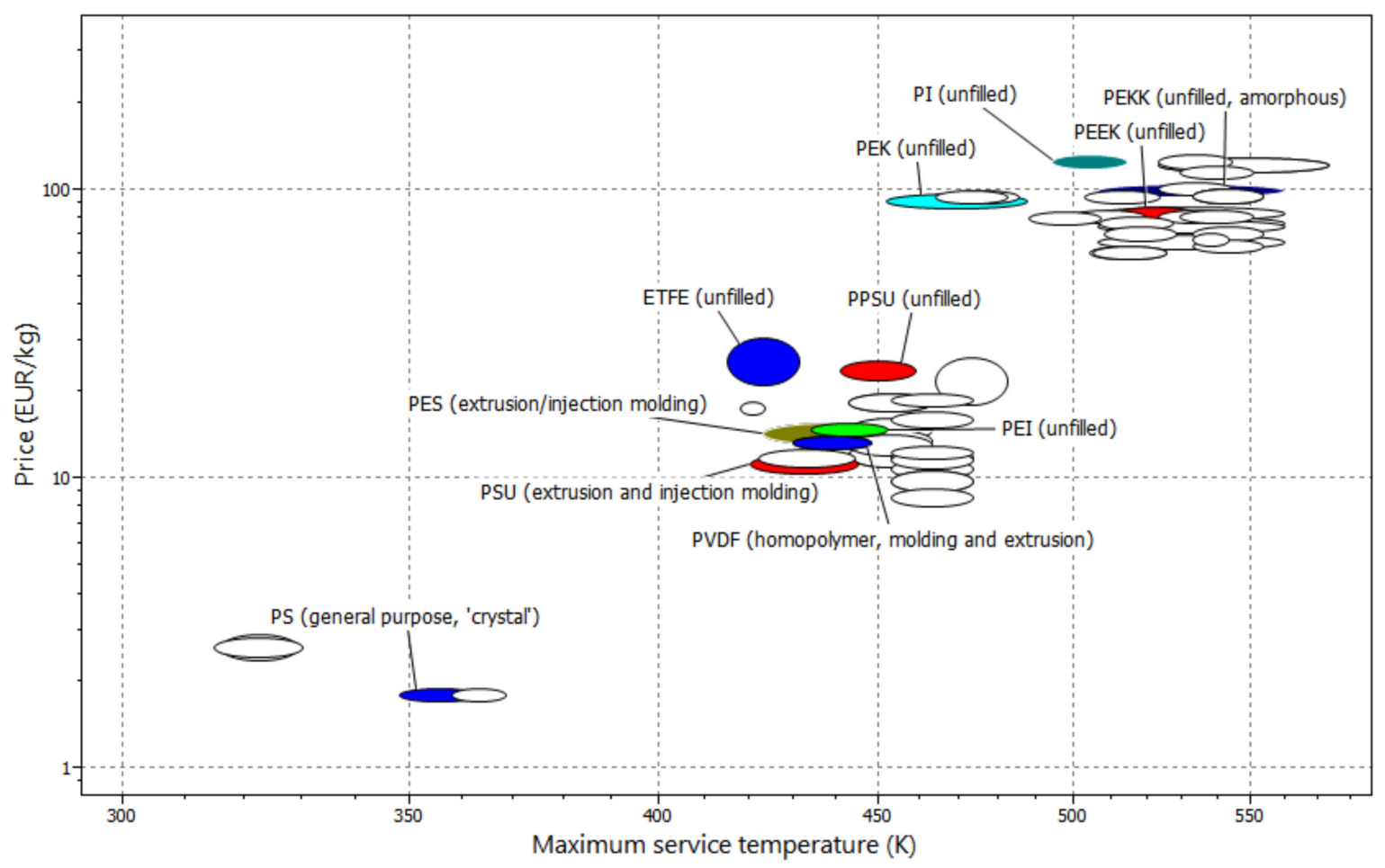

Figure 16. Commercial polymer products: specific costs and maximum service temperature [216].

\section{Conclusions}

By now, numerous flow-battery targeted membranes for the VRFB exist. Most developments show improved VRFB performance when compared to VRFB equipped with reference (Nafion) membranes.

Less often, the stability of membranes is investigated at high cycles of significantly more than 1000. From a technical point of view, information on this has a similar significance as the demonstration of VRFB performance with a new membrane.

When stating costs, only rough estimates can be made usually. The cost of up scaling, e.g., choice and planning of production technology, are often not taken into account. Fundamental examples of the influence of membrane properties on VRFB performance were described in Section 4.2. They can be considered as an approach for the development of membranes based on other, cheaper or chemically more stable polymers.

Results from PFSA membrane modifications lead to the conclusion of improved VRFB performance. These modifications, however, may lead to increased production costs through potential additional steps during manufacturing. PFSA membranes are known for their chemical stability, which was also demonstrated in cycling tests with new "low-cost" or fluorine-free membranes. 1000 and more charge and discharge cycles were achieved 
with MS98 (PVDF, asym, -), MS167 (PEEK, dhe, CEM), MS205 (PSU, sym, AEM), MS254 (QPTM, dho, AEM) and MS270 (PBI, sym, AEM*). A lot of membrane modifications can be found in hydro-carbon-based membranes, where DAPP is investigated in addition to commercially available PEEK or PSU.

Which part of a performance improvement can be attributed to a specific membrane property can generally not be formulated in concrete figures, as these partly influence each other. For example, changes in ion-exchange capacity lead to changes in swelling properties, which affect thickness, water uptake and ultimately selectivity.

For cost optimized VRFB manufacturing, membrane production must be a continuous process on an industrial scale. In addition to investment and operating costs, raw material prices are a large influence for the specific costs of membranes produced in a large scale. In order to determine the material costs, it is therefore necessary to know the material composition of the membrane and the amount of operating materials required for all production steps.

On the basis of the data from this study, we conclude that some suggestions with reference to membrane type for different operating modes of the VRFB can be made. Dense AEM and N-heterocycle-based membranes, especially PBI membranes, are suitable for lowest discharge of the VRFB. Symmetric and asymmetric porous membranes as well as CEM enable VRFB operation at high current densities. AIEM and dense heterogeneous CEM are the choice for operation mode with highest energy efficiency (Table 18). The cost column in Figure 16 shows the specific cost range for the three material groups fluorocarbon, hydro-carbon and $\mathrm{N}$-heterocycle-based membranes. Of course, PVDF and ETFE-based materials are in the cost range of PSU or PES (Figure 16), but the expansive PFSA materials increase the average value. The manufacturing of dense polymer films is generally easier and cheaper than making membranes with a special porosity.

Table 18. Evaluation matrix of polymer membranes (+ less good, ++ good, +++ best).

\begin{tabular}{|c|c|c|c|c|c|}
\hline \multirow{2}{*}{$\begin{array}{l}\text { Membrane Chemistry } \\
\text { and Structure }\end{array}$} & \multicolumn{3}{|c|}{ Efficiency } & \multirow{2}{*}{$\begin{array}{l}\text { Membrane Material } \\
\text { and Structure }\end{array}$} & \multirow{2}{*}{ Cos } \\
\hline & CE & VE & EE & & \\
\hline CEM & + & +++ & ++ & fluoro-carbon & + \\
\hline AEM & +++ & + & ++ & hydro-carbon & +++ \\
\hline AIEM & ++ & ++ & +++ & N-heterocycle & ++ \\
\hline dense & +++ & ++ & +++ & dense & +++ \\
\hline sym & + & +++ & ++ & sym & ++ \\
\hline asym & ++ & ++ & ++ & asym & ++ \\
\hline
\end{tabular}

VRFB performance, high chemical stability and reduced costs will continue to play an important role in future membrane research. For promising membrane developments, long-term cycling experiments are recommended, whereby the membrane is examined before and after with regard to its chemical and structural change.

There is still research potential in the choice of materials for membrane development using polymer products with a price well below $10 \mathrm{EUR} \mathrm{kg}^{-1}$. For lithium-ion batteries a list of different coated porous polyolefin separators was published in 2016 [217]. The Poly(ethylene) (PE), poly(propylene) (PP) and PE/PP-based low-cost separators can be a good starting material for making VRFB membranes, too. In 2020 such a kind of VRFB membrane was made by coating a hydrophilic poly(ethylene) separator with PBI [198].

The influence of the membrane composition regarding proton conductivity and vanadyl permeation is relatively well known, but the influence of the membrane structure is mostly unknown. Future research and development approaches could include the in-depth investigation of membrane structures and their influence on VRFB performance. This is seldom considered regarding ion-exchange membranes, even though dense CEM, AEM or AIEM also have electrolyte-filled pores and channels. 
The use of commercial polymers is just as advantageous as the use of polymerizable monomers. If large production quantities are considered, the question also arises as to what a suitable recycling concept for discarded membranes could look like. If these membranes were to be selected for thermal recycling, fluorine-free materials would lower cost. This should also be taken into account for other membrane additives.

Future efforts to enhance the design of membranes for VRFB could still be the development of new polymer materials as well as manufacturing technology innovations. Generally, some "simple" and fundamental facts should be taken into account, when designing membranes for VRFB-based on polymers:

- Dimensional stability after soaking the dry membrane in battery electrolyte or water is very important to keep the ion channels diameters as small as possible.

- For sulfonated polymers as a proton conductor in the membranes, it should be taken into account that its acidity is dependent on the polymer used and influences the proton conductivity.

- The thickness of the membrane (length of ion channels) should be optimized for high selectivity.

- As many ion channels as possible should be aimed for good conduction between the two half-cells.

The polymer chemistry of a membrane and its interaction with the battery electrolyte not only, but also the membrane morphology allow special membranes for enhanced VRFB performance in low self-discharge, high current density or high energy efficiency mode. The degree of sulfonation and covalent or ionic cross-linking of polymers are important methods to enhance the membrane morphology. This was shown with some membranes mentioned in this study. Polymer cross-linking should be focused when designing membranes with high degree of sulfonation. Additionally, self-ordering polymers, like copolymers or polymers with crystalline proportions could be an option to control membrane morphologies on a molecular scale or to enhance its chemical stability.

Manufacturing technology could include dielectrophoresis units to enhance the design of membranes, too. Dielectrophoresis is a method to separate materials with different dielectric properties. Due to the fact that composite membranes, containing a proton conductor and, e.g., a hydrophobic matrix, consist of materials with different dielectric constants it is possible to align the proton conductor as ion channels between the two surfaces of a flat sheet membrane in an electric field during manufacturing. This might influence proton conductivity and $\mathrm{H}^{+} / \mathrm{V}$ selectivity.

Furthermore, it might be possible to increase the resistance to the highly oxidizing electrolyte of the positive half-cell by additional coating strategies.

Supplementary Materials: https:/ /www.mdpi.com/2077-0375/11/3/214/s1, Figure S1: The water uptake of developed membranes in recent years: (a) fluoro-carbons, (b) hydro-carbons and (c) $N$ heterocycles. Figure S2: The diffusion coefficient ratio of developed membranes in recent years: (a) fluoro-carbons, (b) hydro-carbons and (c) N-heterocycles. Figure S3: The anion exchange capacity of developed membranes in recent years: (a) fluoro-carbons, (b) hydro-carbons and (c) $N$-heterocycles.

Funding: This research was funded by VolkswagenStiftung, grant number 991847. The APC was funded by Ostfalia University of Applied Sciences.

Institutional Review Board Statement: Not applicable.

Informed Consent Statement: Not applicable.

Data Availability Statement: The data presented in this study are cited (reference numbers).

Acknowledgments: We would like to thank the Ministry of Science and Culture of Lower Saxony as well as the Volkswagen Foundation for their support in the context of the project "Future Vehicle Technologies in the Open Region Lab-ZuFOR".

Conflicts of Interest: The authors declare no conflict of interest. 


\section{Abbreviations}

AEM anion exchange membrane

AEM $^{*} \quad$ anion exchange membrane (acidic environment)

AIEM amphoteric ion exchange membrane

AIEM $^{*} \quad$ amphoteric ion exchange membrane (acidic environment)

asym asymmetric

CD current density

$\mathrm{CE}_{\mathrm{H}} \quad$ coulombic efficiency $\left(\geq 100 \mathrm{~mA} \mathrm{~cm}^{-2}\right)$

$\mathrm{CE}_{\mathrm{L}} \quad$ coulombic efficiency $\left(<100 \mathrm{~mA} \mathrm{~cm}^{-2}\right)$

CEM cation exchange membrane

chem chemistry

CL cross-linked

d membrane thickness

$D_{c} \quad$ diffusion coefficient

$D_{\mathrm{r}} \quad$ diffusion coefficient ratio

DAPP diels-Alder Poly(phenylene)

dhe dense and heterogeneous

dho dense and homogeneous

DOE U.S. Department of Energy

EE energy efficiency (charge-discharge)

$E_{r} \quad$ energy efficiency ratio

$\mathrm{EE}_{\mathrm{H}} \quad$ energy efficiency $\left(\geq 100 \mathrm{~mA} \mathrm{~cm}^{-2}\right)$

$\mathrm{EE}_{\mathrm{L}} \quad$ energy efficiency $\left(<100 \mathrm{~mA} \mathrm{~cm}^{-2}\right)$

ETFE poly(ethylene-tetrafluoroethylene)

FPAE fluorinated poly(arylene ether)

IEC ion exchange capacity

Mem membrane

MS membrane sample

PA poly(amide)

PBI poly(benzimidazole)

PEEK poly(ether ether ketone)

PEK poly(ether ketones)

PES poly(ether sulfone)

PF poly(fluorenyle)

PFSA perfluorosulfonic acid

PI poly(imide)

PPE poly(phenylene ether)

PPEK poly(phthalazinone ether ketones)

PPh poly(phenylene)

PPSU poly(phenyl sulfones)

PS poly(styrene)

PSU poly(sulfones)

PTFE poly(tetrafluoroethylene)

pub publication

PVA poly(vinyl alcohol)

PVC poly(vinyl chloride)

PVDF poly(vinylidene fluoride)

Ref reference

struc structure

sym symmertric

$\mathrm{V} \quad$ vanadium

$\mathrm{VE}_{\mathrm{H}} \quad$ voltage efficiency $\left(\geq 100 \mathrm{~mA} \mathrm{~cm}^{-2}\right)$

$\mathrm{VE}_{\mathrm{L}} \quad$ voltage efficiency $\left(<100 \mathrm{~mA} \mathrm{~cm}^{-2}\right)$

VRFB all vanadium redox flow battery

WU water uptake 


\section{References}

1. Gür, T.M. Review of electrical energy storage technologies, materials and systems: Challenges and prospects for large-scale grid storage. Energy Environ. Sci. 2018, 11, 2696-2767. [CrossRef]

2. REN21, Renewables 2020-Global Status Report. 2020. Available online: https://www.ren21.net/reports/global-status-report/ (accessed on 25 February 2021).

3. CellCube. CellCube Reference Project. Potential for improvement of the VRFB. 2020. Available online: https://www.cellcube. com/pellworm (accessed on 25 February 2021).

4. Colthorpe, A. China's Biggest Flow Battery Project so Far Is Underway with Hundreds More Megawatts to Come. 2018. Available online: https: / www.energy-storage.news/news/chinas-biggest-flow-battery-project-so-far-is-underway-with-hundredsmore-m (accessed on 25 February 2021).

5. Vanadiumcorp, Sumitomo Electric 60 Megawatt Hour Vanadium Redox Battery for Hokkaido. 2020. Available online: https: / / www.vanadiumcorp.com/news/industry/sumitomo-electric-60-megawatt-hour-vanadium-redox-battery-for-hokkaido/ (accessed on 25 February 2021).

6. Fraunhofer ICT, Großprojekt RedoxWind. 2020. Available online: https://www.ict.fraunhofer.de/de/komp/ae/RFBWind.html (accessed on 25 February 2021).

7. Storion Energy GmbH, Products. 2020. Available online: http:/ / storion-energy.de/produkte/ (accessed on 25 February 2021).

8. Voltstorage, Vanadium Redox Flow Technology. 2020. Available online: https://voltstorage.com/ (accessed on 25 February 2021).

9. Volterion, Systems. 2020. Available online: https://www.volterion.com/systeme-2/ (accessed on 25 February 2021).

10. Minke, C.; Turek, T. Materials, system designs and modelling approaches in techno-economic assessment of all-vanadium redox flow batteries-A review. J. Power Sources 2018, 376, 66-81. [CrossRef]

11. Li, X.; Zhang, H.; Mai, Z.; Zhang, H.; Vankelecom, I. Ion exchange membranes for vanadium redox flow battery (VRB) applications. Energy Environ. Sci. 2011, 4, 1147. [CrossRef]

12. Schwenzer, B.; Zhang, J.; Kim, S.; Li, L.; Liu, J.; Yang, Z. Membrane Development for Vanadium Redox Flow Batteries. ChemSusChem 2011, 4, 1388-1406. [CrossRef]

13. Prifti, H.; Parasuraman, A.; Winardi, S.; Lim, T.M.; Skyllas-Kazacos, M. Membranes for redox flow battery applications. Membranes 2012, 2, 275-306. [CrossRef] [PubMed]

14. Cha, S.-H. Recent Development of Nanocomposite Membranes for Vanadium Redox Flow Batteries. J. Nanomater. 2015, 1-12. [CrossRef]

15. Parasuraman, A.; Lim, T.M.; Menictas, C.; Skyllas-Kazacos, M. Review of material research and development for vanadium redox flow battery applications. Electrochim. Acta 2013, 101, 27-40. [CrossRef]

16. Varcoe, J.R.; Atanassov, P.; Dekel, D.R.; Herring, A.M.; Hickner, M.A.; Kohl, P.A.; Kucernak, A.R.; Mustain, W.E.; Nijmeijer, K.; Scott, K.; et al. Anion-exchange membranes in electrochemical energy systems. Energy Environ. Sci. 2014, 7, 3135-3191. [CrossRef]

17. Doan, T.N.L.; Hoang, T.K.A.; Chen, P. Recent development of polymer membranes as separators for all-vanadium redox flow batteries. RSC Adv. 2015, 5, 72805-72815. [CrossRef]

18. Maurya, S.; Shin, S.-H.; Kim, Y.; Moon, S.-H. A review on recent developments of anion exchange membranes for fuel cells and redox flow batteries. RSC Adv. 2015, 5, 37206-37230. [CrossRef]

19. Lu, W.; Yuan, Z.; Zhao, Y.; Zhang, H.; Zhang, H.; Li, X. Porous membranes in secondary battery technologies. Chem. Soc. Rev. 2017, 46, 2199-2236. [CrossRef]

20. Ran, J.; Wu, L.; He, Y.; Yang, Z.; Wang, Y.; Jiang, C.; Ge, L.; Bakangura, E.; Xu, T. Ion exchange membranes: New developments and applications. J. Membr. Sci. 2017, 522, 267-291. [CrossRef]

21. Yuan, Z.; Zhang, H.; Li, X. Ion conducting membranes for aqueous flow battery systems. Chem. Commun. 2018, 54, 7570-7588. [CrossRef]

22. Luo, T.; Abdu, S.; Wessling, M. Selectivity of ion exchange membranes: A review. J. Membr. Sci. 2018, 555, 429-454. [CrossRef]

23. Shi, Y.; Eze, C.; Xiong, B.; He, W.; Zhang, H.; Lim, T.M.; Ukil, A.; Zhao, J. Recent development of membrane for vanadium redox flow battery applications: A review. Appl. Energy 2019, 238, 202-224. [CrossRef]

24. Gubler, L. Membranes and separators for redox flow batteries. Curr. Opin. Electrochem. 2019, 18, 31-36. [CrossRef]

25. Tempelman, C.; Jacobs, J.F.; Balzer, R.M.; Degirmenci, V. Membranes for all vanadium redox flow batteries. J. Energy Storage 2020, 32, 101754. [CrossRef]

26. FumaTech GmbH, VRFB Membranes. 2019. Available online: https://www.fumatech.com/EN/Membranes/Batteries/index. html (accessed on 30 November 2019).

27. Zhou, X.L.; Zhao, T.S.; An, L.; Zeng, Y.K.; Zhu, X.B. Performance of a vanadium redox flow battery with a VANADion membrane. Appl. Energy 2016, 180, 353-359. [CrossRef]

28. Mohammadi, T.; Kazacos, M. Modification of anion-exchange membranes for vanadium redox flow battery applications. J. Power Sources 1996, 63, 179-186. [CrossRef]

29. Hwang, G.-J.; Ohya, H. Crosslinking of anion exchange membrane by accelerated electron radiation as a separator for the all-vanadium redox flow battery. J. Membr. Sci. 1997, 132, 55-61. [CrossRef]

30. AGC, Selemion. 2019. Available online: https://www.amp-ionex.com/products/selemion/pdf/selemion.pdf (accessed on 30 November 2019). 
31. Ding, C.; Zhang, H.; Li, X.; Zhang, H.; Yao, C.; Shi, D. Morphology and Electrochemical Properties of Perfluorosulfonic Acid Ionomers for Vanadium Flow Battery Applications: Effect of Side-Chain Length. ChemSusChem 2013, 6, 1262-1269. [CrossRef]

32. Sun, J.; Li, X.; Xi, X.; Lai, Q.; Liu, T.; Zhang, H. The transfer behavior of different ions across anion and cation exchange membranes under vanadium flow battery medium. J. Power Sources 2014, 271, 1-7. [CrossRef]

33. David, O.; Percin, K.; Luo, T.; Gendel, Y.; Wessling, M. Proton-exchange membranes based on sulfonated poly(ether ether ketone)/polyaniline blends for all- and air-vanadium redox flow battery applications. J. Energy Storage 2015, 1, 65-71. [CrossRef]

34. Chromik, A.; Santos, A.R.d.; Turek, T.; Kunz, U.; Häring, T.; Kerres, J. Stability of acid-excess acid-base blend membranes in all-vanadium redox-flow batteries. J. Membr. Sci. 2015, 476, 148-155. [CrossRef]

35. Zeng, L.; Zhao, T.S.; Wei, L.; Zeng, Y.K.; Zhang, Z.H. Highly stable pyridinium-functionalized cross-linked anion exchange membranes for all vanadium redox flow batteries. J. Power Sources 2016, 331, 452-461. [CrossRef]

36. Jiang, B.; Yu, L.; Wu, L.; Mu, D.; Liu, L.; Xi, J.; Qiu, X. Insights into the Impact of the Nafion Membrane Pretreatment Process on Vanadium Flow Battery Performance. ACS Appl. Mater. Interfaces 2016, 8, 12228-12238. [CrossRef] [PubMed]

37. Chen, D.; Hickner, M.A.; Agar, E.; Kumbur, E.C. Selective anion exchange membranes for high coulombic efficiency vanadium redox flow batteries. Electrochem. Commun. 2013, 26, 37-40. [CrossRef]

38. Dai, W.; Shen, Y.; Li, Z.; Yu, L.; Xi, J.; Qiu, X. SPEEK/Graphene oxide nanocomposite membranes with superior cyclability for highly efficient vanadium redox flow battery. J. Mater. Chem. A 2014, 2, 12423-12432. [CrossRef]

39. Chen, D.; Kim, S.; Li, L.; Yang, G.; Hickner, M.A. Stable fluorinated sulfonated poly(arylene ether) membranes for vanadium redox flow batteries. RSC Adv. 2012, 2, 8087. [CrossRef]

40. Jia, C.; Cheng, Y.; Ling, X.; Wei, G.; Liu, J.; Yan, C. Sulfonated Poly(Ether Ether Ketone)/Functionalized Carbon Nanotube Composite Membrane for Vanadium Redox Flow Battery Applications. Electrochim. Acta 2015, 153, 44-48. [CrossRef]

41. Fang, J.; Xu, H.; Wei, X.; Guo, M.; Lu, X.; Lan, C.; Zhang, Y.; Liu, Y.; Peng, T. Preparation and characterization of quaternized poly (2,2,2-trifluoroethyl methacrylate-co-N-vinylimidazole) membrane for vanadium redox flow battery. Polym. Adv. Technol. 2013, 24, 168-173. [CrossRef]

42. Jia, C.; Liu, J.; Yan, C. A multilayered membrane for vanadium redox flow battery. J. Power Sources 2012, 203, 190-194. [CrossRef]

43. Fujimoto, C.; Kim, S.; Stains, R.; Wei, X.; Li, L.; Yang, Z.G. Vanadium redox flow battery efficiency and durability studies of sulfonated Diels Alder poly(phenylene)s. Electrochem. Commun. 2012, 20, 48-51. [CrossRef]

44. Lu, S.; Wu, C.; Liang, D.; Tan, Q.; Xiang, Y. Layer-by-layer self-assembly of Nafion-[CS-PWA] composite membranes with suppressed vanadium ion crossover for vanadium redox flow battery applications. RSC Adv. 2014, 4, 24831-24837. [CrossRef]

45. Jiang, B.; Wu, L.; Yu, L.; Qiu, X.; Xi, J. A comparative study of Nafion series membranes for vanadium redox flow batteries. J. Membr. Sci. 2016, 510, 18-26. [CrossRef]

46. Reed, D.; Thomsen, E.; Wang, W.; Nie, Z.; Li, B.; Wei, X.; Koeppel, B.; Sprenkle, V. Performance of Nafion ${ }^{\circledR}$ N115, Nafion ${ }^{\circledR}$ NR-212, and Nafion ${ }^{\circledR}$ NR-211 in a $1 \mathrm{~kW}$ class all vanadium mixed acid redox flow battery. J. Power Sources 2015, 285, 425-430. [CrossRef]

47. Kim, J.; Jeon, J.-D.; Kwak, S.-Y. Nafion-based composite membrane with a permselective layered silicate layer for vanadium redox flow battery. Electrochem. Commun. 2014, 38, 68-70. [CrossRef]

48. Kim, S.; Yan, J.; Schwenzer, B.; Zhang, J.; Li, L.; Liu, J.; Yang, Z.; Hickner, M.A. Cycling performance and efficiency of sulfonated poly(sulfone) membranes in vanadium redox flow batteries. Electrochem. Commun. 2010, 12, 1650-1653. [CrossRef]

49. Semiz, L.; Sankir, N.D.; Sankir, M. Influence of the basic membrane properties of the disulfonated poly(arylene ether sulfone) copolymer membranes on the vanadium redox flow battery performance. J. Membr. Sci. 2014, 468, 209-215. [CrossRef]

50. Kong, L.; Zheng, L.; Niu, R.; Wang, H.; Shi, H. A sulfonated poly(ether ether ketone)/amine-functionalized graphene oxide hybrid membrane for vanadium redox flow batteries. RSC Adv. 2016, 6, 100262-100270. [CrossRef]

51. Lee, K.J.; Chu, Y.H. Preparation of the graphene oxide (GO)/Nafion composite membrane for the vanadium redox flow battery (VRB) system. Vacuum 2014, 107, 269-276. [CrossRef]

52. Sun, C.-N.; Tang, Z.; Belcher, C.; Zawodzinski, T.A.; Fujimoto, C. Evaluation of Diels-Alder poly(phenylene) anion exchange membranes in all-vanadium redox flow batteries. Electrochem. Commun. 2014, 43, 63-66. [CrossRef]

53. Teng, X.; Dai, J.; Su, J.; Zhu, Y.; Liu, H.; Song, Z. A high performance polytetrafluoroethene/Nafion composite membrane for vanadium redox flow battery application. J. Power Sources 2013, 240, 131-139. [CrossRef]

54. Li, J.; Zhang, Y.; Wang, L. Preparation and characterization of sulfonated polyimide/TiO2 composite membrane for vanadium redox flow battery. J. Solid State Electrochem. 2014, 18, 729-737. [CrossRef]

55. Yan, X.; Zhang, C.; Dai, Y.; Zheng, W.; Ruan, X.; He, G. A novel imidazolium-based amphoteric membrane for high-performance vanadium redox flow battery. J. Membr. Sci. 2017, 544, 98-107. [CrossRef]

56. Li, X.; Santos, A.R.d.; Drache, M.; Ke, X.; Gohs, U.; Turek, T.; Becker, M.; Kunz, U.; Beuermann, S. Polymer electrolyte membranes prepared by pre-irradiation induced graft copolymerization on ETFE for vanadium redox flow battery applications. J. Membr. Sci. 2017, 524, 419-427. [CrossRef]

57. Li, Y.; Lin, X.; Wu, L.; Jiang, C.; Hossain, M.M.; Xu, T. Quaternized membranes bearing zwitterionic groups for vanadium redox flow battery through a green route. J. Membr. Sci. 2015, 483, 60-69. [CrossRef]

58. Li, Z.; Dai, W.; Yu, L.; Xi, J.; Qiu, X.; Chen, L. Sulfonated poly(ether ether ketone)/mesoporous silica hybrid membrane for high performance vanadium redox flow battery. J. Power Sources 2014, 257, 221-229. [CrossRef]

59. Chen, D.; Li, X. Sulfonated poly(ether ether ketone) membranes containing pendent carboxylic acid groups and their application in vanadium flow battery. J. Power Sources 2014, 247, 629-635. [CrossRef] 
60. Li, Z.; Liu, L.; Yu, L.; Wang, L.; Xi, J.; Qiu, X.; Chen, L. Characterization of sulfonated poly(ether ether ketone)/poly(vinylidene fluoride-co-hexafluoropropylene) composite membrane for vanadium redox flow battery application. J. Power Sources 2014, 272, 427-435. [CrossRef]

61. Chen, L.; Zhang, S.; Chen, Y.; Jian, X. Low vanadium ion permeabilities of sulfonated poly(phthalazinone ether ketone)s provide high efficiency and stability for vanadium redox flow batteries. J. Power Sources 2017, 355, 23-30. [CrossRef]

62. Choi, E.M.; Kim, M.K.; Kang, E.T.; Kang, K.B.; Kim, D.S. Perfluorinated polymer for vanadium flow battery. Desalination Water Treat. 2013, 51, 5172-5178. [CrossRef]

63. Liao, J.B.; Lu, M.Z.; Chu, Y.Q.; Wang, J.L. Ultra-low vanadium ion diffusion amphoteric ion-exchange membranes for all-vanadium redox flow batteries. J. Power Sources 2015, 282, 241-247. [CrossRef]

64. Lin, C.-H.; Yang, M.-C.; Wei, H.-J. Amino-silica modified Nafion membrane for vanadium redox flow battery. J. Power Sources 2015, 282, 562-571. [CrossRef]

65. Jang, J.-K.; Kim, T.-H.; Yoon, S.J.; Lee, J.Y.; Lee, J.-C.; Hong, Y.T. Highly proton conductive, dense polybenzimidazole membranes with low permeability to vanadium and enhanced $\mathrm{H}_{2} \mathrm{SO}_{4}$ absorption capability for use in vanadium redox flow batteries. J. Mater. Chem. A 2016, 4, 14342-14355. [CrossRef]

66. Liu, S.; Wang, L.; Ding, Y.; Liu, B.; Han, X.; Song, Y. Novel sulfonated poly (ether ether keton)/polyetherimide acid-base blend membranes for vanadium redox flow battery applications. Electrochim. Acta 2014, 130, 90-96. [CrossRef]

67. Luo, Q.; Zhang, H.; Chen, J.; Qian, P.; Zhai, Y. Modification of Nafion membrane using interfacial polymerization for vanadium redox flow battery applications. J. Membr. Sci. 2008, 311, 98-103. [CrossRef]

68. Luo, X.; Lu, Z.; Xi, J.; Wu, Z.; Zhu, W.; Chen, L.; Qiu, X. Influences of permeation of vanadium ions through PVDF-g-PSSA membranes on performances of vanadium redox flow batteries. J. Phys. Chem. B 2005, 109, 20310-20314. [CrossRef]

69. Kim, S.; Yuk, S.; Kim, H.G.; Choi, C.; Kim, R.; Lee, J.Y.; Hong, Y.T.; Kim, H.-T. A hydrocarbon/Nafion bilayer membrane with a mechanical nano-fastener for vanadium redox flow batteries. J. Mater. Chem. A 2017, 5, 17279-17286. [CrossRef]

70. Maurya, S.; Shin, S.-H.; Lee, J.-Y.; Kim, Y.; Moon, S.-H. Amphoteric nanoporous polybenzimidazole membrane with extremely low crossover for a vanadium redox flow battery. RSC Adv. 2016, 6, 5198-5204. [CrossRef]

71. Kondratenko, M.S.; Karpushkin, E.A.; Gvozdik, N.A.; Gallyamov, M.O.; Stevenson, K.J.; Sergeyev, V.G. Influence of aminosilane precursor concentration on physicochemical properties of composite Nafion membranes for vanadium redox flow battery applications. J. Power Sources 2017, 340, 32-39. [CrossRef]

72. Niu, R.; Kong, L.; Zheng, L.; Wang, H.; Shi, H. Novel graphitic carbon nitride nanosheets/sulfonated poly(ether ether ketone) acid-base hybrid membrane for vanadium redox flow battery. J. Membr. Sci. 2017, 525, 220-228. [CrossRef]

73. Leung, P.K.; Xu, Q.; Zhao, T.S.; Zeng, L.; Zhang, C. Preparation of silica nanocomposite anion-exchange membranes with low vanadium-ion crossover for vanadium redox flow batteries. Electrochim. Acta 2013, 105, 584-592. [CrossRef]

74. Pu, Y.; Huang, X.; Yang, P.; Zhou, Y.; Xuan, S.; Zhang, Y. Effect of non-sulfonated diamine monomer on branched sulfonated polyimide membrane for vanadium redox flow battery application. Electrochim. Acta 2017, 241, 50-62. [CrossRef]

75. Li, J.; Liu, S.; He, Z.; Zhou, Z. Semi-fluorinated sulfonated polyimide membranes with enhanced proton selectivity and stability for vanadium redox flow batteries. Electrochim. Acta 2016, 216, 320-331. [CrossRef]

76. Teng, X.; Zhao, Y.; Xi, J.; Wu, Z.; Qiu, X.; Chen, L. Nafion/organic silica modified TiO2 composite membrane for vanadium redox flow battery via in situ sol-gel reactions. J. Membr. Sci. 2009, 341, 149-154. [CrossRef]

77. Wang, N.; Peng, S.; Lu, D.; Liu, S.; Liu, Y.; Huang, K. Nafion/TiO2 hybrid membrane fabricated via hydrothermal method for vanadium redox battery. J. Solid State Electrochem. 2012, 16, 1577-1584. [CrossRef]

78. Li, J.; Liu, S.; He, Z.; Zhou, Z. A novel branched side-chain-type sulfonated polyimide membrane with flexible sulfoalkyl pendants and trifluoromethyl groups for vanadium redox flow batteries. J. Power Sources 2017, 347, 114-126. [CrossRef]

79. Wang, N.; Peng, S.; Wang, H.; Li, Y.; Liu, S.; Liu, Y. SPPEK/WO3 hybrid membrane fabricated via hydrothermal method for vanadium redox flow battery. Electrochem. Commun. 2012, 17, 30-33. [CrossRef]

80. Wang, N.; Yu, J.; Zhou, Z.; Fang, D.; Liu, S.; Liu, Y. SPPEK/TPA composite membrane as a separator of vanadium redox flow battery. J. Membr. Sci. 2013, 437, 114-121. [CrossRef]

81. Li, Y.; Li, X.; Cao, J.; Xu, W.; Zhang, H. Composite porous membranes with an ultrathin selective layer for vanadium flow batteries. Chem. Commun. 2014, 50, 4596-4599. [CrossRef]

82. Xi, J.; Dai, W.; Yu, L. Polydopamine coated SPEEK membrane for a vanadium redox flow battery. RSC Adv. 2015, 5, 33400-33406. [CrossRef]

83. Mai, Z.; Zhang, H.; Li, X.; Bi, C.; Dai, H. Sulfonated poly(tetramethydiphenyl ether ether ketone) membranes for vanadium redox flow battery application. J. Power Sources 2011, 196, 482-487. [CrossRef]

84. Xi, J.; Li, Z.; Yu, L.; Yin, B.; Wang, L.; Liu, L.; Qiu, X.; Chen, L. Effect of degree of sulfonation and casting solvent on sulfonated poly(ether ether ketone) membrane for vanadium redox flow battery. J. Power Sources 2015, 285, 195-204. [CrossRef]

85. Xi, J.; Wu, Z.; Teng, X.; Zhao, Y.; Chen, L.; Qiu, X. Self-assembled polyelectrolyte multilayer modified Nafion membrane with suppressed vanadium ion crossover for vanadium redox flow batteries. J. Mater. Chem. 2008, 18, 1232. [CrossRef]

86. Seepana, M.M.; Pandey, J.; Shukla, A. Design and synthesis of highly stable poly(tetrafluoroethylene)-zirconium phosphate (PTFE-ZrP) ion-exchange membrane for vanadium redox flow battery (VRFB). Ionics 2017, 23, 1471-1480. [CrossRef]

87. Xia, Z.; Ying, L.; Fang, J.; Du, Y.-Y.; Zhang, W.-M.; Guo, X.; Yin, J. Preparation of covalently cross-linked sulfonated polybenzimidazole membranes for vanadium redox flow battery applications. J. Membr. Sci. 2017, 525, 229-239. [CrossRef] 
88. Wei, W.; Zhang, H.; Li, X.; Mai, Z.; Zhang, H. Poly(tetrafluoroethylene) reinforced sulfonated poly(ether ether ketone) membranes for vanadium redox flow battery application. J. Power Sources 2012, 208, 421-425. [CrossRef]

89. Xie, W.; Darling, R.M.; Perry, M.L. Processing and Pretreatment Effects on Vanadium Transport in Nafion Membranes. J. Electrochem. Soc. 2016, 163, A5084-A5089. [CrossRef]

90. Yin, B.; Li, Z.; Dai, W.; Wang, L.; Yu, L.; Xi, J. Highly branched sulfonated poly(fluorenyl ether ketone sulfone)s membrane for energy efficient vanadium redox flow battery. J. Power Sources 2015, 285, 109-118. [CrossRef]

91. Wei, X.; Nie, Z.; Luo, Q.; Li, B.; Chen, B.; Simmons, K.; Sprenkle, V.; Wang, W. Nanoporous Polytetrafluoroethylene/Silica Composite Separator as a High-Performance All-Vanadium Redox Flow Battery Membrane. Adv. Energy Mater. 2013, 3, 1215-1220. [CrossRef]

92. Yin, B.; Yu, L.; Jiang, B.; Wang, L.; Xi, J. Nano oxides incorporated sulfonated poly(ether ether ketone) membranes with improved selectivity and stability for vanadium redox flow battery. J. Solid State Electrochem. 2016, 20, 1271-1283. [CrossRef]

93. Xi, X.; Ding, C.; Zhang, H.; Li, X.; Cheng, Y.; Zhang, H. Solvent responsive silica composite nanofiltration membrane with controlled pores and improved ion selectivity for vanadium flow battery application. J. Power Sources 2015, 274, 1126-1134 [CrossRef]

94. Yue, M.; Zhang, Y.; Wang, L. Sulfonated polyimide/chitosan composite membrane for vanadium redox flow battery: Membrane preparation, characterization, and single cell performance. J. Appl. Polym. Sci. 2013, 127, 4150-4159. [CrossRef]

95. Yuan, Z.; Dai, Q.; Zhao, Y.; Lu, W.; Li, X.; Zhang, H. Polypyrrole modified porous poly(ether sulfone) membranes with high performance for vanadium flow batteries. J. Mater. Chem. A 2016, 4, 12955-12962. [CrossRef]

96. Zhang, B.; Zhang, E.; Wang, G.; Yu, P.; Zhao, Q.; Yao, F. Poly(phenyl sulfone) anion exchange membranes with pyridinium groups for vanadium redox flow battery applications. J. Power Sources 2015, 282, 328-334. [CrossRef]

97. Yuan, Z.; Zhu, X.; Li, M.; Lu, W.; Li, X.; Zhang, H. A Highly Ion-Selective Zeolite Flake Layer on Porous Membranes for Flow Battery Applications. Angew. Chem. (Int. Ed. Engl.) 2016, 55, 3058-3062. [CrossRef]

98. Zhang, B.; Zhang, S.; Weng, Z.; Wang, G.; Zhang, E.; Yu, P.; Chen, X.; Wang, X. Quaternized adamantane-containing poly(aryl ether ketone) anion exchange membranes for vanadium redox flow battery applications. J. Power Sources 2016, 325, 801-807. [CrossRef]

99. Zhang, H.; Ding, C.; Cao, J.; Xu, W.; Li, X.; Zhang, H. A novel solvent-template method to manufacture nano-scale porous membranes for vanadium flow battery applications. J. Mater. Chem. A 2014, 2, 9524. [CrossRef]

100. Zhang, B.; Zhang, S.; Xing, D.; Han, R.; Yin, C.; Jian, X. Quaternized poly(phthalazinone ether ketone ketone) anion exchange membrane with low permeability of vanadium ions for vanadium redox flow battery application. J. Power Sources 2012, 217, 296-302. [CrossRef]

101. Zhang, H.; Zhang, H.; Li, X.; Mai, Z.; Wei, W.; Li, Y. Crosslinkable sulfonated poly (diallyl-bisphenol ether ether ketone) membranes for vanadium redox flow battery application. J. Power Sources 2012, 217, 309-315. [CrossRef]

102. Zhao, Y.; Li, M.; Yuan, Z.; Li, X.; Zhang, H.; Vankelecom, I.F.J. Advanced Charged Sponge-Like Membrane with Ultrahigh Stability and Selectivity for Vanadium Flow Batteries. Adv. Funct. Mater. 2016, 26, 210-218. [CrossRef]

103. Zhang, S.; Zhang, B.; Xing, D.; Jian, X. Poly(phthalazinone ether ketone ketone) anion exchange membranes with pyridinium as ion exchange groups for vanadium redox flow battery applications. J. Mater. Chem. A 2013, 1, 12246. [CrossRef]

104. Zhang, Y.; Li, J.; Wang, L.; Zhang, S. Sulfonated polyimide/AlOOH composite membranes with decreased vanadium permeability and increased stability for vanadium redox flow battery. J. Solid State Electrochem. 2014, 18, 3479-3490. [CrossRef]

105. Zhang, Y.; Chen, J.; Zhang, J.; Wang, G.; Zhang, J.; Zhu, X.; Wang, R. Sulfonated poly(ether ether ketone)/poly(vinylidene fluoride)/tungstophosphoric acid membrane for vanadium redox flow battery application. High Perform. Polym. 2016, 28, 735-740. [CrossRef]

106. Strathmann, H. Introduction to Membrane Science and Technology; Wiley-VCH: Weinheim, Germany, 2011.

107. Melin, T.; Rautenbach, R. Membranverfahren: Grundlagen der Modul- und Anlagenauslegung, 3rd ed.; Springer: Berlin/Heidelberg, Germany, 2007.

108. Nunes, S.P.; Peinemann, K.-V. Membrane Technology. In The Chemical Industry; Wiley-VCH: Hoboken, NJ, USA, 2007.

109. Krebs, F.C. Fabrication and processing of polymer solar cells: A review of printing and coating techniques. Solar Energy Mater. Solar Cells 2009, 93, 394-412. [CrossRef]

110. Toshikatsu, S. Ion Exchange Membranes; Royal Society of Chemistry: Cambridge, UK, 2007.

111. Mohammadi, T.; Skyllas-Kazacos, M. Preparation of sulfonated composite membrane for vanadium redox flow battery applications. J. Membr. Sci. 1995, 107, 35-45. [CrossRef]

112. Teng, X.; Dai, J.; Su, J.; Yin, G. Modification of Nafion membrane using fluorocarbon surfactant for all vanadium redox flow battery. J. Membr. Sci. 2015, 476, 20-29. [CrossRef]

113. Chen, D.; Hickner, M.A.; Agar, E.; Kumbur, E.C. Optimizing membrane thickness for vanadium redox flow batteries. J. Membr. Sci. 2013, 437, 108-113. [CrossRef]

114. Chen, D.; Hickner, M.A.; Agar, E.; Kumbur, E.C. Optimized anion exchange membranes for vanadium redox flow batteries. ACS Appl. Mater. Interfaces 2013, 5, 7559-7566. [CrossRef] [PubMed]

115. Teng, X.; Dai, J.; Bi, F.; Yin, G. Ultra-thin polytetrafluoroethene/Nafion/silica composite membrane with high performance for vanadium redox flow battery. J. Power Sources 2014, 272, 113-120. [CrossRef] 
116. Kim, J.; Lee, Y.; Jeon, J.-D.; Kwak, S.-Y. Ion-exchange composite membranes pore-filled with sulfonated poly(ether ether ketone) and Engelhard titanosilicate-10 for improved performance of vanadium redox flow batteries. J. Power Sources 2018, 383, 1-9. [CrossRef]

117. Cao, J.; Zhang, H.; Xu, W.; Li, X. Poly(vinylidene fluoride) porous membranes precipitated in water/ethanol dual-coagulation bath. J. Power Sources 2014, 249, 84-91. [CrossRef]

118. Wei, W.; Zhang, H.; Li, X.; Zhang, H.; Li, Y.; Vankelecom, I. Hydrophobic asymmetric ultrafiltration PVDF membranes: An alternative separator for VFB with excellent stability. Phys. Chem. Chem. Phys. PCCP 2013, 15, 1766-1771. [CrossRef] [PubMed]

119. Cao, J.; Yuan, Z.; Li, X.; Xu, W.; Zhang, H. Hydrophilic poly(vinylidene fluoride) porous membrane with well connected ion transport networks for vanadium flow battery. J. Power Sources 2015, 298, 228-235. [CrossRef]

120. Ma, J.; Wang, Y.; Peng, J.; Qiu, J.; Xu, L.; Li, J.; Zhai, M. Designing a new process to prepare amphoteric ion exchange membrane with well-distributed grafted chains for vanadium redox flow battery. J. Membr. Sci. 2012, 419-420, 1-8. [CrossRef]

121. Hu, G.; Wang, Y.; Ma, J.; Qiu, J.; Peng, J.; Li, J.; Zhai, M. A novel amphoteric ion exchange membrane synthesized by radiationinduced grafting $\alpha$-methylstyrene and $\mathrm{N}, \mathrm{N}$-dimethylaminoethyl methacrylate for vanadium redox flow battery application. $J$. Membr. Sci. 2012, 407-408, 184-192. [CrossRef]

122. Qiu, J.; Zhao, L.; Zhai, M.; Ni, J.; Zhou, H.; Peng, J.; Li, J.; Wei, G. Pre-irradiation grafting of styrene and maleic anhydride onto PVDF membrane and subsequent sulfonation for application in vanadium redox batteries. J. Power Sources 2008, 177, 617-623. [CrossRef]

123. Qiu, J.; Zhang, J.; Chen, J.; Peng, J.; Xu, L.; Zhai, M.; Li, J.; Wei, G. Amphoteric ion exchange membrane synthesized by radiationinduced graft copolymerization of styrene and dimethylaminoethyl methacrylate into PVDF film for vanadium redox flow battery applications. J. Membr. Sci. 2009, 334, 9-15. [CrossRef]

124. Ling, L.; Xiao, M.; Han, D.; Ren, S.; Wang, S.; Meng, Y. Porous composite membrane of PVDF/Sulfonic silica with high ion selectivity for vanadium redox flow battery. J. Membr. Sci. 2019, 585, 230-237. [CrossRef]

125. Rajput, A.; Khan, H.; Raj, S.K.; Kothandaraman, R.; Kulshrestha, V. Styrene- co -DVB grafted PVDF proton exchange membranes for vanadium redox flow battery applications. Mater. Adv. 2020, 1, 1930-1938. [CrossRef]

126. Qiu, J.; Zhai, M.; Chen, J.; Wang, Y.; Peng, J.; Xu, L.; Li, J.; Wei, G. Performance of vanadium redox flow battery with a novel amphoteric ion exchange membrane synthesized by two-step grafting method. J. Membr. Sci. 2009, 342, 215-220. [CrossRef]

127. Qiu, J.; Li, M.; Ni, J.; Zhai, M.; Peng, J.; Xu, L.; Zhou, H.; Li, J.; Wei, G. Preparation of ETFE-based anion exchange membrane to reduce permeability of vanadium ions in vanadium redox battery. J. Membr. Sci. 2007, 297, 174-180. [CrossRef]

128. Nibel, O.; Rojek, T.; Schmidt, T.J.; Gubler, L. Amphoteric Ion-Exchange Membranes with Significantly Improved Vanadium Barrier Properties for All-Vanadium Redox Flow Batteries. ChemSusChem 2017, 10, 2767-2777. [CrossRef]

129. Mallinson, S.L.; Varcoe, J.R.; Slade, R.C. Examination of Amine-Functionalised Anion-Exchange Membranes for Possible Use in the All-Vanadium Redox Flow Battery. Electrochim. Acta 2014, 140, 145-151. [CrossRef]

130. Yao, C.; Zhang, H.; Liu, T.; Li, X.; Liu, Z. Cell architecture upswing based on catalyst coated membrane (CCM) for vanadium flow battery. J. Power Sources 2013, 237, 19-25. [CrossRef]

131. Teng, X.; Zhao, Y.; Xi, J.; Wu, Z.; Qiu, X.; Chen, L. Nafion/organically modified silicate hybrids membrane for vanadium redox flow battery. J. Power Sources 2009, 189, 1240-1246. [CrossRef]

132. Zhang, L.; Ling, L.; Xiao, M.; Han, D.; Wang, S.; Meng, Y. Effectively suppressing vanadium permeation in vanadium redox flow battery application with modified Nafion membrane with nacre-like nanoarchitectures. J. Power Sources 2017, 352, 111-117. [CrossRef]

133. Mai, Z.; Zhang, H.; Li, X.; Xiao, S.; Zhang, H. Nafion/polyvinylidene fluoride blend membranes with improved ion selectivity for vanadium redox flow battery application. J. Power Sources 2011, 196, 5737-5741. [CrossRef]

134. Teng, X.; Lei, J.; Gu, X.; Dai, J.; Zhu, Y.; Li, F. Nafion-sulfonated organosilica composite membrane for all vanadium redox flow battery. Ionics 2012, 18, 513-521. [CrossRef]

135. Vijayakumar, M.; Schwenzer, B.; Kim, S.; Yang, Z.; Thevuthasan, S.; Liu, J.; Graff, G.L.; Hu, J. Investigation of local environments in Nafion-SiO(2) composite membranes used in vanadium redox flow batteries. Solid State Nucl. Magn. Reson. 2012, 42, 71-80. [CrossRef]

136. Luo, Q.; Zhang, H.; Chen, J.; You, D.; Sun, C.; Zhang, Y. Preparation and characterization of Nafion/SPEEK layered composite membrane and its application in vanadium redox flow battery. J. Membr. Sci. 2008, 325, 553-558. [CrossRef]

137. Aziz, M.A.; Shanmugam, S. Zirconium oxide nanotube-Nafion composite as high performance membrane for all vanadium redox flow battery. J. Power Sources 2017, 337, 36-44. [CrossRef]

138. Chen, D.; Kim, S.; Sprenkle, V.; Hickner, M.A. Composite blend polymer membranes with increased proton selectivity and lifetime for vanadium redox flow batteries. J. Power Sources 2013, 231, 301-306. [CrossRef]

139. Pezeshki, A.M.; Tang, Z.J.; Fujimoto, C.; Sun, C.-N.; Mench, M.M.; Zawodzinski, T.A. Full Cell Study of Diels Alder Poly(phenylene) Anion and Cation Exchange Membranes in Vanadium Redox Flow Batteries. J. Electrochem. Soc. 2016, 163, A5154-A5162. [CrossRef]

140. Largier, T.D.; Cornelius, C.J. Random quaternary ammonium Diels-Alder poly(phenylene) copolymers for improved vanadium redox flow batteries. J. Power Sources 2017, 352, 149-155. [CrossRef]

141. Wang, T.; Jeon, J.Y.; Han, J.; Kim, J.H.; Bae, C.; Kim, S. Poly(terphenylene) anion exchange membranes with high conductivity and low vanadium permeability for vanadium redox flow batteries (VRFBs). J. Membr. Sci. 2020, 598, 117665. [CrossRef] 
142. Mu, D.; Yu, L.; Liu, L.; Xi, J. Rice Paper Reinforced Sulfonated Poly(ether ether ketone) as Low-Cost Membrane for Vanadium Flow Batteries. ACS Sustain. Chem. Eng. 2017, 5, 2437-2444. [CrossRef]

143. Macksasitorn, S.; Changkhamchom, S.; Sirivat, A.; Siemanond, K. Sulfonated poly(ether ether ketone) and sulfonated poly(1,4phenylene ether ether sulfone) membranes for vanadium redox flow batteries. High Perform. Polym. 2012, 24, 603-608. [CrossRef]

144. Yu, L.; Xi, J. Durable and Efficient PTFE Sandwiched SPEEK Membrane for Vanadium Flow Batteries. ACS Appl. Mater. Interfaces 2016, 8, 23425-23430. [CrossRef]

145. Dai, W.; Yu, L.; Li, Z.; Yan, J.; Liu, L.; Xi, J.; Qiu, X. Sulfonated Poly(Ether Ether Ketone)/Graphene composite membrane for vanadium redox flow battery. Electrochim. Acta 2014, 132, 200-207. [CrossRef]

146. Wang, F.; Wang, G.; Zhang, J.; Li, B.; Zhang, J.; Deng, J.; Chen, J.; Wang, R. Novel sulfonated poly(ether ether ketone)/oxidized g-C $3 \mathrm{~N} 4$ composite membrane for vanadium redox flow battery applications. J. Electroanal. Chem. 2017, 797, 107-112. [CrossRef]

147. Li, Z.; Xi, J.; Zhou, H.; Liu, L.; Wu, Z.; Qiu, X.; Chen, L. Preparation and characterization of sulfonated poly(ether ether ketone)/poly(vinylidene fluoride) blend membrane for vanadium redox flow battery application. J. Power Sources 2013, 237, 132-140. [CrossRef]

148. Hyeon, D.H.; Chun, J.H.; Lee, C.H.; Jung, H.C.; Kim, S.H. Composite membranes based on sulfonated poly(ether ether ketone) and $\mathrm{SiO} 2$ for a vanadium redox flow battery. Korean J. Chem. Eng. 2015, 32, 1554-1563. [CrossRef]

149. Ji, Y.; Tay, Z.Y.; Li, S.F.Y. Highly selective sulfonated poly(ether ether ketone)/titanium oxide composite membranes for vanadium redox flow batteries. J. Membr. Sci. 2017, 539, 197-205. [CrossRef]

150. Jia, C.; Liu, J.; Yan, C. A significantly improved membrane for vanadium redox flow battery. J. Power Sources 2010, 195, 4380-4383. [CrossRef]

151. Li, Z.; Dai, W.; Yu, L.; Liu, L.; Xi, J.; Qiu, X.; Chen, L. Properties investigation of sulfonated poly(ether ether ketone)/polyacrylonitrile acid-base blend membrane for vanadium redox flow battery application. ACS Appl. Mater. Interfaces 2014, 6, 18885-18893. [CrossRef]

152. Wang, F.; Sylvia, J.M.; Jacob, M.M.; Peramunage, D. Amphiphilic block copolymer membrane for vanadium redox flow battery. J. Power Sources 2013, 242, 575-580. [CrossRef]

153. Yun, S.; Parrondo, J.; Ramani, V. Derivatized cardo-polyetherketone anion exchange membranes for all-vanadium redox flow batteries. J. Mater. Chem. A 2014, 2, 6605-6615. [CrossRef]

154. Aziz, M.A.; Shanmugam, S. Sulfonated graphene oxide-decorated block copolymer as a proton-exchange membrane: Improving the ion selectivity for all-vanadium redox flow batteries. J. Mater. Chem. A 2018, 6, 17740-17750. [CrossRef]

155. Zhang, Y.; Wang, H.; Liu, B.; Shi, J.; Zhang, J.; Shi, H. An ultra-high ion selective hybrid proton exchange membrane incorporated with zwitterion-decorated graphene oxide for vanadium redox flow batteries. J. Mater. Chem. A 2019, 7, 12669-12680. [CrossRef]

156. Quan, Y.; Wang, G.; Li, A.; Wei, X.; Li, F.; Zhang, J.; Chen, J.; Wang, R. Novel sulfonated poly(ether ether ketone)/triphenylamine hybrid membrane for vanadium redox flow battery applications. RSC Adv. 2019, 9, 3838-3846. [CrossRef]

157. Ye, J.; Cheng, Y.; Sun, L.; Ding, M.; Wu, C.; Yuan, D.; Zhao, X.; Xiang, C.; Jia, C. A green SPEEK/lignin composite membrane with high ion selectivity for vanadium redox flow battery. J. Membr. Sci. 2019, 572, 110-118. [CrossRef]

158. Bhushan, M.; Kumar, S.; Singh, A.K.; Shahi, V.K. High-performance membrane for vanadium redox flow batteries: Cross-linked poly(ether ether ketone) grafted with sulfonic acid groups via the spacer. J. Membr. Sci. 2019, 583, 1-8. [CrossRef]

159. Zhang, B.; Wang, Q.; Guan, S.; Weng, Z.; Zhang, E.; Wang, G.; Zhang, Z.; Hu, J.; Zhang, S. High performance membranes based on new 2-adamantane containing poly(aryl ether ketone) for vanadium redox flow battery applications. J. Power Sources 2018, 399, 18-25. [CrossRef]

160. Kumar, S.; Bhushan, M.; Shahi, V.K. Cross-linked amphoteric membrane: Sulphonated poly(ether ether ketone) grafted with 2,4,6-tris(dimethylaminomethyl)phenol using functionalized side chain spacers for vanadium redox flow battery. J. Power Sources 2020, 448, 227358. [CrossRef]

161. Hossain, S.I.; Aziz, M.A.; Han, D.; Selvam, P.; Shanmugam, S. Fabrication of SPAEK-cerium zirconium oxide nanotube composite membrane with outstanding performance and durability for vanadium redox flow batteries. J. Mater. Chem. A 2018, 6, 20205-20213. [CrossRef]

162. Zhang, F.; Zhang, H.; Qu, C. A Dication Cross-Linked Composite Anion-Exchange Membrane for All-Vanadium Flow Battery Applications. ChemSusChem 2013, 6, 2290-2298. [CrossRef]

163. Zeng, L.; Zhao, T.S.; Wei, L.; Zeng, Y.K.; Zhang, Z.H. Polyvinylpyrrolidone-based semi-interpenetrating polymer networks as highly selective and chemically stable membranes for all vanadium redox flow batteries. J. Power Sources 2016, 327, $374-383$. [CrossRef]

164. Li, Y.; Zhang, H.; Zhang, H.; Cao, J.; Xu, W.; Li, X. Hydrophilic porous poly(sulfone) membranes modified by UV-initiated polymerization for vanadium flow battery application. J. Membr. Sci. 2014, 454, 478-487. [CrossRef]

165. Zhang, H.; Zhang, H.; Zhang, F.; Li, X.; Li, Y.; Vankelecom, I. Advanced charged membranes with highly symmetric spongy structures for vanadium flow battery application. Energy Environ. Sci. 2013, 6, 776. [CrossRef]

166. Jung, M.J.; Parrondo, J.; Arges, C.G.; Ramani, V. Polysulfone-based anion exchange membranes demonstrate excellent chemical stability and performance for the all-vanadium redox flow battery. J. Mater. Chem. A 2013, 1, 10458. [CrossRef]

167. Xing, Y.; Liu, L.; Wang, C.; Li, N. Side-chain-type anion exchange membranes for vanadium flow battery: Properties and degradation mechanism. J. Mater. Chem. A 2018, 6, 22778-22789. [CrossRef] 
168. Zhang, Y.; Zheng, L.; Liu, B.; Wang, H.; Shi, H. Sulfonated polysulfone proton exchange membrane influenced by a varied sulfonation degree for vanadium redox flow battery. J. Membr. Sci. 2019, 584, 173-180. [CrossRef]

169. Liu, B.; Zhang, Y.; Jiang, Y.; Qian, P.; Shi, H. High performance acid-base composite membranes from sulfonated polysulfone containing graphitic carbon nitride nanosheets for vanadium redox flow battery. J. Membr. Sci. 2019, 591, 117332. [CrossRef]

170. Chen, D.; Hickner, M.A.; Agar, E.; Kumbur, E.C. Anion Exchange Membranes for Vanadium Redox Flow Batteries. ECS Trans. 2013, 53, 83-89. [CrossRef]

171. Li, Y.; Zhang, H.; Li, X.; Zhang, H.; Wei, W. Porous poly (ether sulfone) membranes with tunable morphology. J. Power Sources 2013, 233, 202-208. [CrossRef]

172. Chen, D.; Li, D.; Li, X. Highly symmetric spongy porous poly(ether sulfone) membranes with selective open-cells for vanadium flow battery application. RSC Adv. 2016, 6, 87104-87109. [CrossRef]

173. Xu, W.; Li, X.; Cao, J.; Yuan, Z.; Zhang, H. Morphology and performance of poly(ether sulfone)/sulfonated poly(ether ether ketone) blend porous membranes for vanadium flow battery application. RSC Adv. 2014, 4, 40400-40406. [CrossRef]

174. Chen, D.; Li, D.; Li, X. Hierarchical porous poly (ether sulfone) membranes with excellent capacity retention for vanadium flow battery application. J. Power Sources 2017, 353, 11-18. [CrossRef]

175. Zhao, Y.; Yuan, Z.; Lu, W.; Li, X.; Zhang, H. The porous membrane with tunable performance for vanadium flow battery. J. Power Sources 2017, 342, 327-334. [CrossRef]

176. Ling, X.; Jia, C.; Liu, J.; Yan, C. Preparation and characterization of sulfonated poly(ether sulfone)/sulfonated poly(ether ether ketone) blend membrane for vanadium redox flow battery. J. Membr. Sci. 2012, 415-416, 306-312. [CrossRef]

177. Zhou, X.; Xue, R.; Zhong, Y.; Zhang, Y.; Jiang, F. Asymmetric porous membranes with ultra-high ion selectivity for vanadium redox flow batteries. J. Membr. Sci. 2020, 595, 117614. [CrossRef]

178. Teng, X.; Guo, Y.; Liu, D.; Li, G.; Yu, C.; Dai, J. A polydopamine-coated polyamide thin film composite membrane with enhanced selectivity and stability for vanadium redox flow battery. J. Membr. Sci. 2020, 601, 117906. [CrossRef]

179. Chen, D.; Hickner, M.A.; Wang, S.; Pan, J.; Xiao, M.; Meng, Y. Directly fluorinated polyaromatic composite membranes for vanadium redox flow batteries. J. Membr. Sci. 2012, 415-416, 139-144. [CrossRef]

180. Chen, D.; Wang, S.; Xiao, M.; Meng, Y. Preparation and properties of sulfonated poly(fluorenyl ether ketone) membrane for vanadium redox flow battery application. J. Power Sources 2010, 195, 2089-2095. [CrossRef]

181. Pan, J.; Wang, S.; Xiao, M.; Hickner, M.; Meng, Y. Layered zirconium phosphate sulfophenylphosphonates reinforced sulfonated poly (fluorenyl ether ketone) hybrid membranes with high proton conductivity and low vanadium ion permeability. J. Membr. Sci. 2013, 443, 19-27. [CrossRef]

182. Wang, Y.; Wang, S.; Xiao, M.; Han, D.; Hickner, M.A.; Meng, Y. Layer-by-layer self-assembly of PDDA/PSS-SPFEK composite membrane with low vanadium permeability for vanadium redox flow battery. RSC Adv. 2013, 3, 15467. [CrossRef]

183. Wang, Y.; Wang, S.; Xiao, M.; Han, D.; Meng, Y. Preparation and characterization of a novel layer-by-layer porous composite membrane for vanadium redox flow battery (VRB) applications. Int. J. Hydrogen Energy 2014, 39, 16088-16095. [CrossRef]

184. Wang, Y.; Wang, S.; Xiao, M.; Song, S.; Han, D.; Hickner, M.A.; Meng, Y. Amphoteric ion exchange membrane synthesized by direct polymerization for vanadium redox flow battery application. Int. J. Hydrogen Energy 2014, 39, 16123-16131. [CrossRef]

185. Thong, P.T.; Sadhasivam, T.; Lim, H.; Jin, C.-S.; Ryi, S.-K.; Park, W.; Kim, H.T.; Roh, S.-H.; Jung, H.-Y. High Oxidizing Stability and Ion Selectivity of Hybrid Polymer Electrolyte Membrane for Improving Electrochemical Performance in Vanadium Redox Flow Battery. J. Electrochem. Soc. 2018, 165, A2321-A2329. [CrossRef]

186. Hwang, C.W.; Park, H.-M.; Oh, C.M.; Hwang, T.S.; Shim, J.; Jin, C.-S. Synthesis and characterization of vinylimidazole-cotrifluoroethylmethacrylate-co-divinylbenzene anion-exchange membrane for all-vanadium redox flow battery. J. Membr. Sci. 2014, 468, 98-106. [CrossRef]

187. Park, S.-G.; Kwak, N.-S.; Hwang, C.W.; Park, H.-M.; Hwang, T.S. Synthesis and characteristics of aminated vinylbenzyl chlorideco-styrene-co-hydroxyethyl acrylate anion-exchange membrane for redox flow battery applications. J. Membr. Sci. 2012, 423-424, 429-437. [CrossRef]

188. Wei, X.; Nie, Z.; Luo, Q.; Li, B.; Sprenkle, V.; Wang, W. Polyvinyl Chloride/Silica Nanoporous Composite Separator for All-Vanadium Redox Flow Battery Applications. J. Electrochem. Soc. 2013, 160, A1215-A1218. [CrossRef]

189. Pandey, J.; Tankal, B.R. Performance of the vanadium redox-flow battery (VRB) for Si-PWA/PVA nanocomposite membrane. J. Solid State Electrochem. 2016, 20, 2259-2265. [CrossRef]

190. Kwak, N.-S.; Sim, J.B.; Koo, J.S.; Hwang, T.S.; Kim, Y.T. Synthesis and characteristics of a cross-linked DMSIP-co-HDO-co-MA ion-exchange membrane for redox flow battery applications. J. Membr. Sci. 2013, 430, 252-262. [CrossRef]

191. Chae, I.S.; Luo, T.; Moon, G.H.; Ogieglo, W.; Kang, Y.S.; Wessling, M. Ultra-High Proton/Vanadium Selectivity for Hydrophobic Polymer Membranes with Intrinsic Nanopores for Redox Flow Battery. Adv. Energy Mater. 2016, 6, 1600517. [CrossRef]

192. Noh, C.; Jung, M.; Henkensmeier, D.; Nam, S.W.; Kwon, Y. Vanadium Redox Flow Batteries Using meta-Polybenzimidazole-Based Membranes of Different Thicknesses. ACS Appl. Mater. Interfaces 2017, 9, 36799-36809. [CrossRef]

193. Luo, T.; David, O.; Gendel, Y.; Wessling, M. Porous poly(benzimidazole) membrane for all vanadium redox flow battery. J. Power Sources 2016, 312, 45-54. [CrossRef]

194. Peng, S.; Yan, X.; Wu, X.; Zhang, D.; Luo, Y.; Su, L.; He, G. Thin skinned asymmetric polybenzimidazole membranes with readily tunable morphologies for high-performance vanadium flow batteries. RSC Adv. 2017, 7, 1852-1862. [CrossRef] 
195. Yuan, Z.; Duan, Y.; Zhang, H.; Li, X.; Zhang, H.; Vankelecom, I. Advanced porous membranes with ultra-high selectivity and stability for vanadium flow batteries. Energy Environ. Sci. 2016, 9, 441-447. [CrossRef]

196. Ahn, S.M.; Jeong, H.Y.; Jang, J.-K.; Lee, J.Y.; So, S.; Kim, Y.J.; Hong, Y.T.; Kim, T.-H. Polybenzimidazole/Nafion hybrid membrane with improved chemical stability for vanadium redox flow battery application. RSC Adv. 2018, 8, 25304-25312. [CrossRef]

197. Hu, L.; Gao, L.; Yan, X.; Zheng, W.; Dai, Y.; Hao, C.; Wu, X.; He, G. Proton delivery through a dynamic 3D H-bond network constructed from dense hydroxyls for advanced ion-selective membranes. J. Mater. Chem. A 2019, 7, 15137-15144. [CrossRef]

198. Gubler, L.; Vonlanthen, D.; Schneider, A.; Oldenburg, F.J. Composite Membranes Containing a Porous Separator and a Polybenzimidazole Thin Film for Vanadium Redox Flow Batteries. J. Electrochem. Soc. 2020, 167, 100502. [CrossRef]

199. Bülbül, E.; Atanasov, V.; Mehlhorn, M.; Bürger, M.; Chromik, A.; Häring, T.; Kerres, J. Highly phosphonated polypentafluorostyrene blended with polybenzimidazole: Application in vanadium redox flow battery. J. Membr. Sci. 2019, 570-571, 194-203. [CrossRef]

200. Lee, W.; Jung, M.; Serhiichuk, D.; Noh, C.; Gupta, G.; Harms, C.; Kwon, Y.; Henkensmeier, D. Layered composite membranes based on porous PVDF coated with a thin, dense PBI layer for vanadium redox flow batteries. J. Membr. Sci. 2019, 591, 117333. [CrossRef]

201. Wang, L.; Pingitore, A.T.; Xie, W.; Yang, Z.; Perry, M.L.; Benicewicz, B.C. Sulfonated PBI Gel Membranes for Redox Flow Batteries. J. Electrochem. Soc. 2019, 166, A1449-A1455. [CrossRef]

202. Wei, H.; Liu, Y.; Xu, W.; Liu, J.; Yan, C.; Che, X.; Yang, J.; Tong, J.; Xiao, W. Communication-Polyethylene/PBI Pore-Filling Composite Membrane for High Performance Vanadium Redox Flow Battery. J. Electrochem. Soc. 2019, 166, A3207-A3209. [CrossRef]

203. Zhang, S.H.; Zhang, B.G.; Jian, X.G. Preparation and Properties of Poly (phthalazinone Ether Ketone) Based Anion Exchange Membranes for Vanadium Redox Flow Battery. AMR 2013, 773, 171-174. [CrossRef]

204. Zhang, S.; Zhang, B.; Zhao, G.; Jian, X. Anion exchange membranes from brominated poly(aryl ether ketone) containing 3,5-dimethyl phthalazinone moieties for vanadium redox flow batteries. J. Mater. Chem. A 2014, 2, 3083. [CrossRef]

205. Wang, N.; Peng, S.; Li, Y.; Wang, H.; Liu, S.; Liu, Y. Sulfonated poly(phthalazinone ether sulfone) membrane as a separator of vanadium redox flow battery. J. Solid State Electrochem. 2012, 16, 2169-2177. [CrossRef]

206. Huang, X.; Pu, Y.; Zhou, Y.; Zhang, Y.; Zhang, H. In-situ and ex-situ degradation of sulfonated polyimide membrane for vanadium redox flow battery application. J. Membr. Sci. 2017, 526, 281-292. [CrossRef]

207. Li, J.; Yuan, X.; Liu, S.; He, Z.; Zhou, Z.; Li, A. A Low-Cost and High-Performance Sulfonated Polyimide Proton-Conductive Membrane for Vanadium Redox Flow/Static Batteries. ACS Appl. Mater. Interfaces 2017, 9, 32643-32651. [CrossRef]

208. Zhang, Y.; Li, J.; Zhang, H.; Zhang, S.; Huang, X. Sulfonated polyimide membranes with different non-sulfonated diamines for vanadium redox battery applications. Electrochim. Acta 2014, 150, 114-122. [CrossRef]

209. Li, J.; Zhang, Y.; Zhang, S.; Huang, X. Sulfonated polyimide/s-MoS2 composite membrane with high proton selectivity and good stability for vanadium redox flow battery. J. Membr. Sci. 2015, 490, 179-189. [CrossRef]

210. Cao, L.; Sun, Q.; Gao, Y.; Liu, L.; Shi, H. Novel acid-base hybrid membrane based on amine-functionalized reduced graphene oxide and sulfonated polyimide for vanadium redox flow battery. Electrochim. Acta 2015, 158, 24-34. [CrossRef]

211. Cao, L.; Kong, L.; Kong, L.; Zhang, X.; Shi, H. Novel sulfonated polyimide/zwitterionic polymer-functionalized graphene oxide hybrid membranes for vanadium redox flow battery. J. Power Sources 2015, 299, 255-264. [CrossRef]

212. Li, J.; Zhang, Y.; Zhang, S.; Huang, X.; Wang, L. Novel sulfonated polyimide/ZrO 2 composite membrane as a separator of vanadium redox flow battery. Polym. Adv. Technol. 2014, 25, 1610-1615. [CrossRef]

213. Düerkop, D.; Widdecke, H.; dos Santos, U.K. Polyimide Membrane for Vanadium Redox-Flow Battery. In Proceedings of the IFBF The International Flow Battery Forum-Conference Papers, Manchester, UK, 27-29 June 2014; pp. 70-71.

214. Minke, C.; Kunz, U.; Turek, T. Techno-economic assessment of novel vanadium redox flow batteries with large-area cells. J. Power Sources 2017, 361, 105-114. [CrossRef]

215. Minke, C.; Turek, T. Economics of vanadium redox flow battery membranes. J. Power Sources 2015, 286, 247-257. [CrossRef]

216. CES. CES Selector Software; Granta Design Limited: Cambridge, UK, 2018; Available online: www.grantadesign.com (accessed on 17 January 2018).

217. Xiang, Y.; Li, J.; Lei, J.; Liu, D.; Xie, Z.; Qu, D.; Li, K.; Deng, T.; Tang, H. Advanced Separators for Lithium-Ion and Lithium-Sulfur Batteries: A Review of Recent Progress. ChemSusChem 2016, 9, 3023-3039. [CrossRef] [PubMed] 